\section{SANDIA REPORT}

SAND96-0121 • UC-700

Unlimited Release

Printed January 1996

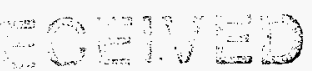

FEB 27 ถึ:

OSTI

\title{
Magnetic Field Diffusion Modeling of a Small Enclosed Firing System
}

Larry K. Warne, Kimball O. Merewether

\section{Prepared by}

Sandia National Laboratories

Albuquerque, New Mexico 87185 and Livermore, California 94550

for the United States Department of Energy

under Contract DE-AC04-94AL85000

Approved for public release; distribution is unlimited.
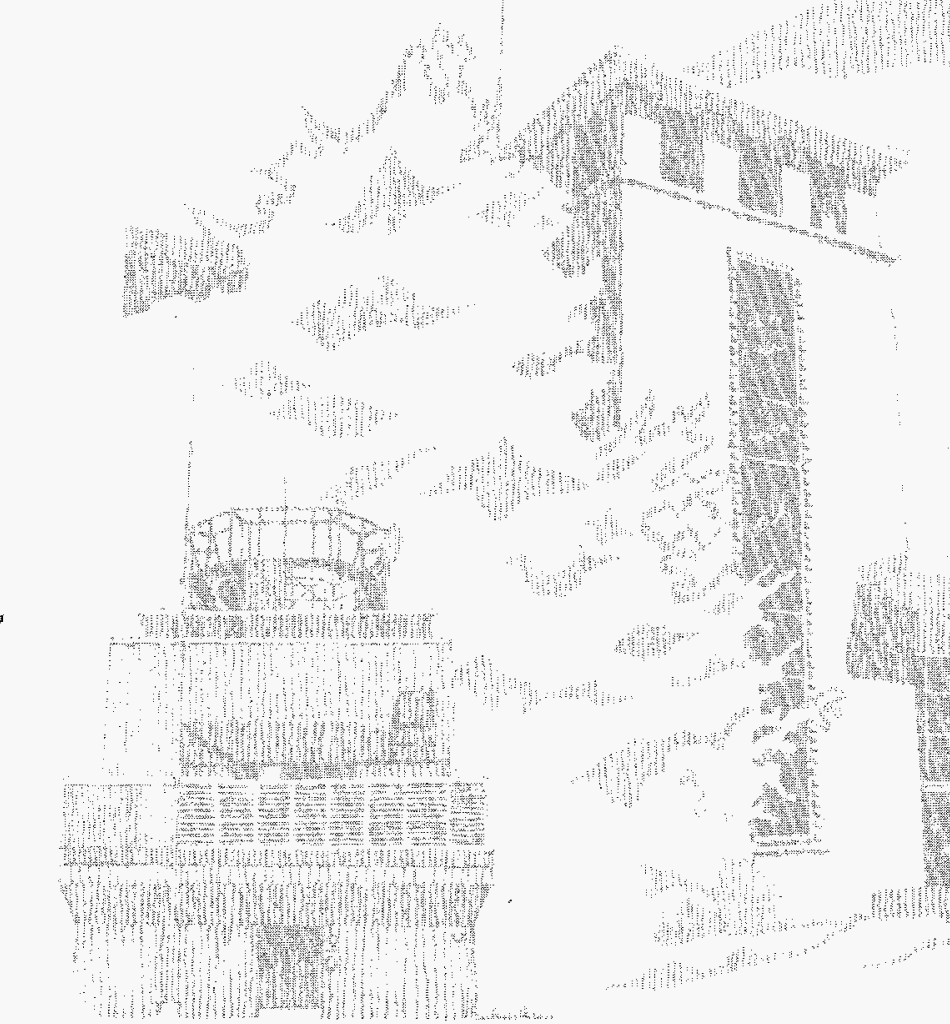
Issued by Sandia National Laboratories, operated for the United States Department of Energy by Sandia Corporation.

NOTICE: This report was prepared as an account of work sponsored by an agency of the United States Government. Neither the United States Government nor any agency thereof, nor any of their employees, nor any of their contractors, subcontractors, or their employees, makes any warranty, express or implied, or assumes any legal liability or responsibility for the accuracy, completeness, or usefulness of any information, apparatus, product, or process disclosed, or represents that its use would not infringe privately owned rights. Reference herein to any specific commercial product, process, or service by trade name, trademark, manufacturer, or otherwise, does not necessarily constitute or imply its endorsement, recommendation, or favoring by the United States Government, any agency thereof or any of their contractors or subcontractors. The views and opinions expressed herein do not necessarily state or reflect those of the United States Government, any agency thereof or any of their contractors.

Printed in the United States of America. This report has been reproduced directly from the best available copy.

Available to DOE and DOE contractors from Office of Scientific and Technical Information PO Box 62

Oak Ridge, TN 37831

Prices available from (615) 576-8401, FTS 626-8401

Available to the public from National Technical Information Service

US Department of Commerce

5285 Port Royal Rd

Springfield, VA 22161

NTIS price codes Printed copy: A05

Microfiche copy: A01 


\section{DISCLAIMIER}

Portions of this document may be illegible in electronic image products. Images are produced from the best available original document. 
SAND96-0121

Distribution

Unlimited Release

Printed January 1996

Category UC-700

\title{
Magnetic Field Diffusion Modeling of a Small Enclosed Firing System
}

\author{
Larry K. Warne and Kimball O. Merewether \\ Electromagnetic Analysis and Test Department \\ Sandia National Laboratories \\ Albuquerque, New Mexico 87185-0865
}

\begin{abstract}
Intense magnetic fields exist in the immediate vicinity of a lightning strike (and near power lines). Conducting barriers increase the rise time (and thus decrease the rise rate) interior to the barrier, but typically do not prevent penetration of the magnetic field, since the lightning current fall time may be larger than the barrier diffusion time. Thus, substantial energy is present in the interior field, although the degradation of rise rate makes it more difficult to couple into electrical circuits. This report assesses the threat posed by the diffusive magnetic field to interior components and wire loops (where voltages are induced). Analytical and numerical bounding analyses are carried out on a pill box shaped conducting barrier to develop estimates for the worst case magnetic field threats inside the system. Worst case induced voltages and energies are estimated and compared with threshold charge voltages and energies on the output capacitor of the system. Variability of these quantities with respect to design parameters are indicated. The interior magnetic field and induced voltage estimates given in this report can be used as excitations for more detailed interior and component models.
\end{abstract}




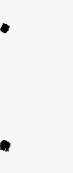




\section{Magnetic Field Diffusion Modeling of a Small Enclosed Firing System}

\section{EXECUTIVE SUMMARY}

A bounding analysis was used to assess the threat posed by the time changing magnetic field of a lightning strike (or a power line) to a small enclosed firing system. The question being addressed is whether the penetrant time changing magnetic field can couple to the interior circuit wiring or the transformer and charge the output capacitor of the firing system. Figure 0 contains a summary presentation of this analysis, which will be discussed here.

Figure $0 \mathrm{a}$ is the title vu-graph. Figure $0 \mathrm{~b}$ gives the objectives. The analysis approach, which included the use of canonical models and two-dimensional numerical models to determine the penetrant magnetic fields inside the firing system, is listed in Figure 0c. Loop areas are assumed (which bound the actual circuit loop areas in the device) in order to estimate induced voltages from the penetrant magnetic field. The lightning current threat is described by Figure 0d which includes the $1 \%$ worst case lightning parameters (which are typically used for safety analyses) of peak current, fall time to half peak, peak rise rate, and number of return stoke currents (each return stroke current being described by the inverse exponential fit function given in this Figure) per lightning flash. A sketch and list of dimensions of the firing system geometry is given in Figure $0 \mathrm{e}$. The enclosure, which the lightning magnetic field must penetrate, is a continuous metallic pill box shaped shield with a $1 \mathrm{~mm}$ thickness (except for a small disc of thickness $0.1 \mathrm{~mm}$ ) made out of either stainless steel or aluminum. Figure of shows a caricature of the magnetic flux coupling problem interior to the pill box shield; coupling to the primary and secondary circuits, as well as direct transformer coupling are the primary concerns. The capacitor thresholds are given along with an upper bound estimate of the primary and secondary circuit loop areas (which is taken as the interior diameter times the interior height). The threshold levels are $V_{\text {out }}=700$ volts, $W_{\text {out }}=18.4$ $\mathrm{mJ}$, which are used for comparison throughout the report; the no-go levels are $V_{\text {out }}=500$ volts, $W_{\text {out }}=9.4 \mathrm{~mJ}$, which are also important for comparison in certain safety studies.

Figure $0 \mathrm{~g}$ illustrates that the apparent worst case threat results not from direct attachment but from an adjacent exterior wire carrying the lightning current. Theoretically the worst case involves a single wall thickness spacing of $1 \mathrm{~mm}$ between the lightning current and the interior pick-up circuit loop. A practical worst case roughly involves additional $1 \mathrm{~mm}$ spacings to the interior circuit loop and to the exterior current (noting that the exterior wire must be insulated). Figure $0 \mathrm{~h}$ illustrates the simpler and very useful uniform field excitation which is considered first; a uniform field approximation is reasonable if the exterior wire is say one pill box radius away (it is also interesting that the uniform field results can be scaled up to closer spacings and yield reasonable agreement with more rigorous treatments). Figure $0 \mathrm{i}$ gives the analytical results for the uniform field excitation. Since the lightning current fall time is considerably longer than the shield diffusion time constant $\tau_{d}$ we can approximate the lightning magnetic field by a step function. The analytical solution from the electromagnetic pulse literature [Lee] for the interior magnetic field given impulse excitation, thus yields the time derivative of the interior magnetic field for step excitation. This result was arrived at by considering the solution of canonical enclosures such as planes, cylinders, and spheres. Figure $0 \mathrm{j}$ gives the numerical values this model predicts for the pill box enclosure. The uniform field induced voltages are relatively small (although for stainless steel if such a voltage were induced in the primary circuit the step up of the transformer, with a turns ratio of 40 , would be of concern).

Figure 0k considers deviations of the actual pill box shield geometry from the canonical shapes used to derive the uniform field results. The rise rate of the penetrant magnetic field in the vicinity 
of a corner is enhanced (the time harmonic problem with electrically thick shield was considered by [Kaden]). This results in our case in a factor of $2-4$ enhancement over the nominal level of the penetrant field rise rate. However because the enhancement is confined to the immediate vicinity of the corners and the fact that the circuits are somewhat removed from the corner region, and also that the transformer windings are not effectively linked by this enhanced field, previous results are still valid. The second deviation from the canonical shields results from the thin foil region in the enclosure. The foil was modeled by using impedance loaded aperture theory which results in an equivalent static magnetic dipole placed in the center of the aperture. Summation of the dipole images in the top and bottom walls of the enclosure gives an equivalent magnetic line dipole which yields the interior fields. The rate of change of the field at twice the foil radius (which is a reasonable distance to describe the circuit board and transformer fields) is less than the direct penetration for the stainless steel enclosure and is more than twice the direct penetration (but still relatively small) for the aluminum enclosure. It is important to note that since the line source of lightning current is constrained to be above the top surface (the opposite side with respect to the foil) of the pill box shield, as the line source is moved closer to the enclosure (to consider the worst case excitation) the foil excitation does not significantly increase (relative to the nominal uniform field value) whereas excitation for the direct penetration does significantly increase. Thus the foil deviation does not appear to be a concern.

A two dimensional finite element code was used as a check on the previous analytical results for the uniform field excitation. Figure 01 shows the cross section of the pill box shield that was gridded in the finite element model. The shaded region was treated as free space or air as well as a perfect magnetic conductor (PMC) (to model a high permeability transformer). The field point locations are noted. (Note that the placement of the foil region adjacent to the interior wall rather than the exterior wall has little impact on the results.) Figure $0 \mathrm{~m}$ shows the time derivative of the interior magnetic field with stainless steel shield, air core, and $x$ polarized incident field. The corner enhancement and foil penetrations are clearly visible. The analytic value $520 \mathrm{kA} / \mathrm{m} / \mu \mathrm{s}$ is quite representative of the fields throughout the remainder of the enclosure. Figure 0n shows the corresponding interior field.

Figure 0o considers the nearby wire excitation. The rigorous solution, when the distance between wire and interior pick-up loop is one wall thickness $1 \mathrm{~mm}$ (the interior loop is taken to have a length $\ell$ equal to the interior diameter of the enclosure), yields induced voltages large enough to be of direct concern in the stainless steel case. The more practical worst case when the distance between exterior wire and interior loop is $3 \mathrm{~mm}$ still yields voltages of some concern (noting the transformer step up) to rule out a problem in the stainless steel case. (This $3 \mathrm{~mm}$ problem was solved using the thin sheet impedance approximation which is thought to slightly overestimate the true voltage.) It is interesting that if we scale up the uniform field results to represent the more closely spaced wire position, the induced voltages are similar to these more rigorous models.

Direct transformer coupling is considered in Figure 0p. Because the interior field rises to values far in excess of ferrite saturation induction levels, we treat the transformer as an air core. The open circuit voltage of the secondary is thus estimated for uniform field excitation. The stainless steel enclosure yields open circuit voltages far in excess of the output capacitor threshold. The practical worst case line source excitation (again using the thin sheet impedance model and maximizing the vertical field at the center of the transformer as a function of horizontal position of the exterior line source) yields nearly three times the uniform field levels. Figure 0q shows a crude secondary circuit of the saturated transformer with primary shorted; this is used to obtain a more realistic estimate of the energy delivered to the output capacitor (energy levels below what the open circuit voltage would imply). The finite length solenoid is used to estimate the leakage inductance of the secondary (we have ignored the small inner primary core area). Because the oscillation period of 
the inductor-capacitor circuit is long compared to the decay time of the induced voltage we can use an approximation to obtain the output energy. Thus for a single return stroke the output energy is above threshold. More detailed modeling of the transformer and actual primary loads could be incorporated in the future in an attempt to reduce this energy calculation below threshold (however multiple return strokes must be considered).

Figure 0r shows an alternative approach involving a simple energy calculation for a solenoid model of the transformer secondary. The interior field is taken to be the same as the exterior field (certainly this level is reached for the stainless steel enclosure). The energy stored in the inductance of the secondary coil is thus proportional to the energy density of the field times the volume of the solenoid; this again yields a level above threshold. Of course this approach incorporates no information about circuit impedance mismatches (the same energy result would be obtained even for quasistatic fields, which would in reality induce little voltage in the output circuit).

Figure 0 s gives estimates for the practical worst case induced voltage in the circuit loops, as well the open circuit secondary voltage from direct transformer coupling, when an additional $3 \mathrm{~mm}$ thick outer shield wall is present. Only the stainless steel case is considered. The direct transformer open circuit voltage is slightly above threshold. However it should be noted that the approximate impedance solution overestimates the induced voltage; the true solution for the open circuit voltage is thus thought to be at or below the voltage threshold. When using worst case models, such as discussed here, large margins are not required.

Figure $0 t$ briefly considers the power line current threat, which is thought to be less severe than lightning.

A summary of the aluminum shield results is given in Figure 0u. A summary of the stainless steel shield results is given in Figure 0v. A summary of the stainless steel shield results, incorporating an outer stainless steel shield wall, is given in Figure 0w.

Figures $0 \mathrm{x}$ and $0 \mathrm{y}$ give conclusions. 


\section{Magnetic Field Diffusion}

\section{Modeling of a Small Enclosed Firing System}

Larry K. Warne, Kimball O. Merewether, Mark J. De Spain Sandia National Laboratories Albuquerque, NM 87185-0865

October 17, 1995 


\section{Objectives}

- Relatively modest $(\$ 30 \mathrm{~K})$ analysis program to determine

- Fields within enclosure

- Bounds on threat to system (compared to threshold voltage and energy)

- From magnetic fields of

- Lightning currents

- Power line currents 
- Apply bounding canonical models to estimate interior fields

- Corroborate with 2D FEM numerical results

- Use bounding loop areas to estimate open circuit voltage drives and bounds on available energies

- Compare with output capacitor thresholds 


\section{Lightning Threat}

Safety studies use $1 \%$ worst-case parameters

- Peak amplitude

$$
I_{0} \approx 200 \mathrm{kA} \quad \text { [Cianos and Pierce] }
$$

- Fall time to half peak amplitude

$$
t_{h} \approx 200 \mu s
$$

[Cianos and Pierce]

- Peak rise rate

$$
\frac{\partial}{\partial t} \mathbf{l} \approx 400 \mathrm{kA} / \mu \mathrm{s} \quad \text { [Uman and Fisher] }
$$

- Number of return strokes/lightning flash

$$
\begin{aligned}
& \mathrm{N}_{\mathbf{r}} \approx 12 \quad \text { [Cianos and } \\
& \mathrm{I}(\mathrm{t}) \approx \frac{\mathrm{I} \mathrm{I}_{0} \mathrm{U}^{\alpha t}}{\mathrm{I}+\mathrm{e}^{\beta\left(\mathrm{t}-\mathrm{t}_{\mathrm{p}}\right)}}, \quad \mathrm{U}=\beta(\beta-\alpha)^{\alpha / \beta-1} \alpha^{-\alpha / \beta} \mathrm{e}^{-\alpha \mathrm{t}_{\mathrm{p}}} \\
& \beta \approx 8 \mu \mathrm{s}^{-1}, \alpha \approx 7.99653 \mu \mathrm{s}^{-1}, \mathrm{t}_{\mathrm{p}} \approx 2 \mu \mathrm{s}
\end{aligned}
$$

[Cianos and Pierce] 


\section{Enclosure Geometry}
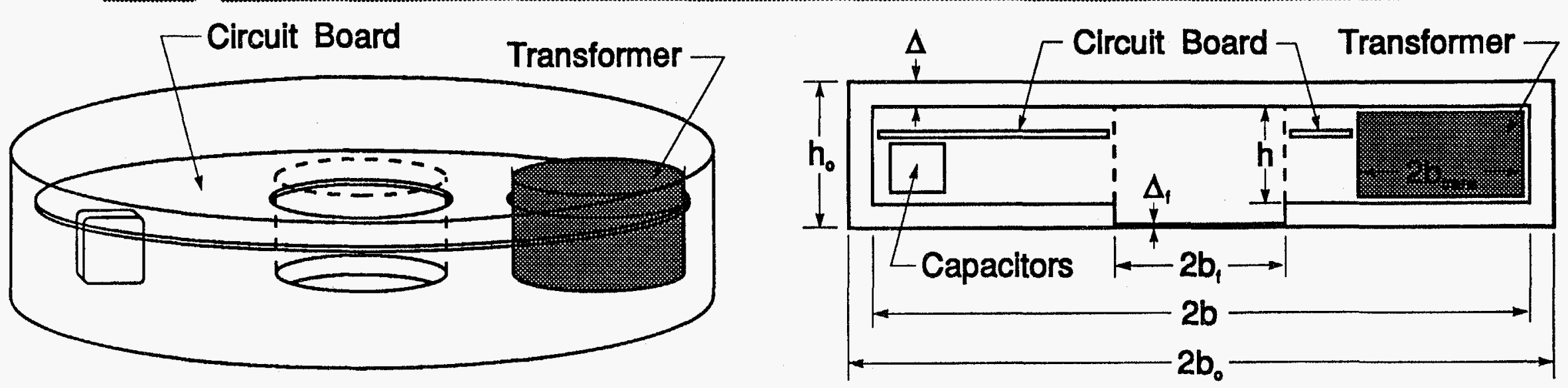

$$
\begin{aligned}
b_{\circ} & \approx 14.5 \mathrm{~mm} \\
b & \approx 13.5 \mathrm{~mm} \\
\Delta & \approx 1 \mathrm{~mm}
\end{aligned}
$$$$
b_{\text {trans }} \approx 3.5 \mathrm{~mm}
$$

$\mathrm{h}_{\mathrm{o}} \approx 6 \mathrm{~mm}$

Enclosure Materials

$$
\mathrm{h} \approx 4 \mathrm{~mm}
$$$$
\mu=\mu_{0}=4 \pi \times 10^{-7} \mathrm{H} / \mathrm{m}
$$

$\Delta_{f} \approx 0.1 \mathrm{~mm}$ 304 stainless steel $\sigma \approx 1.4 \times 10^{6} \mathrm{~S} / \mathrm{m}$

$b_{f} \approx 3.5 \mathrm{~mm}$ 6061 Aluminum $\sigma \approx 2.6 \times 10^{7} \mathrm{~S} / \mathrm{m}$ 


\section{Caricature of Interior Circuitry}

\section{$\Phi=$ magnetic flux}
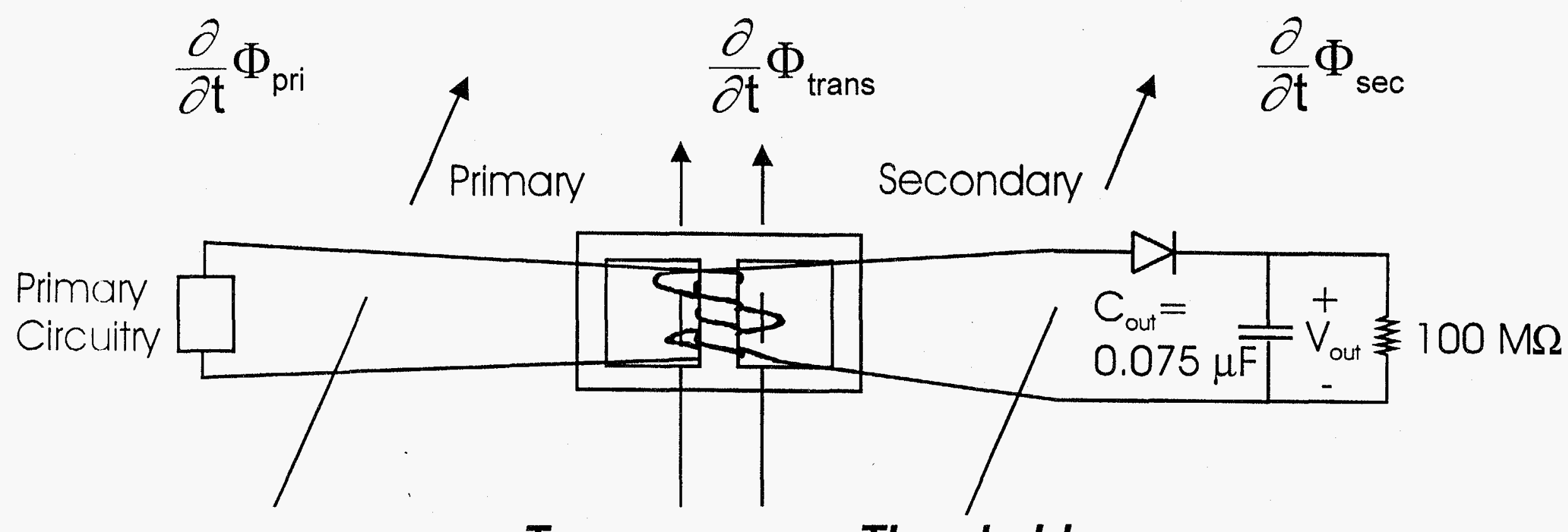

Turns

Thresholds

$$
\begin{array}{cc}
N_{\text {sec }}=400 & V_{\text {out }}=700 \text { volts } \\
N_{\text {pri }}=10 & W_{\text {out }}=18.4 \mathrm{~mJ}
\end{array}
$$

Assume primary and secondary circuit loop areas bounded by $A=2 b h=1.1 \mathrm{~cm}^{2}$ (interior housing diameter times height) 
Insi llated wire

carrying lightning

current I $(t)$

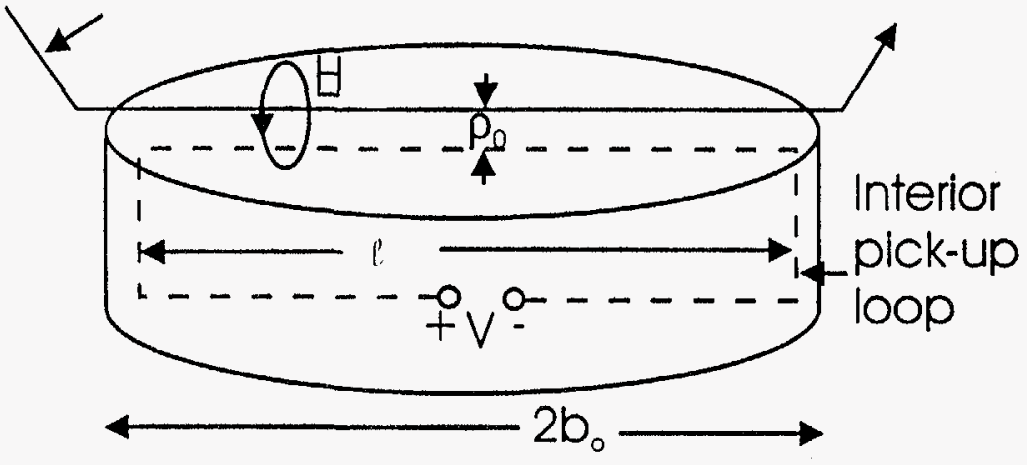

Adjacent wire worst case

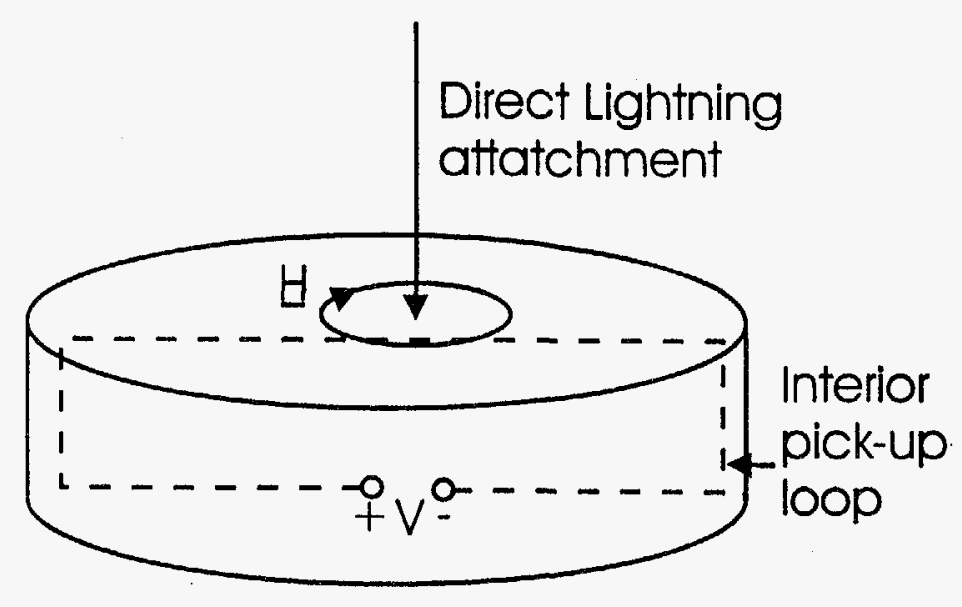

Direct attachment less severe

- Theoretical worst case $\rho_{0}=\Delta \approx 1 \mathrm{~mm}$ (Take $\ell \approx 2 b \approx 27 \mathrm{~mm}$ )

- Practical worst case $p_{0} \approx 3 \mathrm{~mm}$ (circuit board clearance + insulated wire radius) 


\section{Lightning Magnetic Field Threat (continued)}

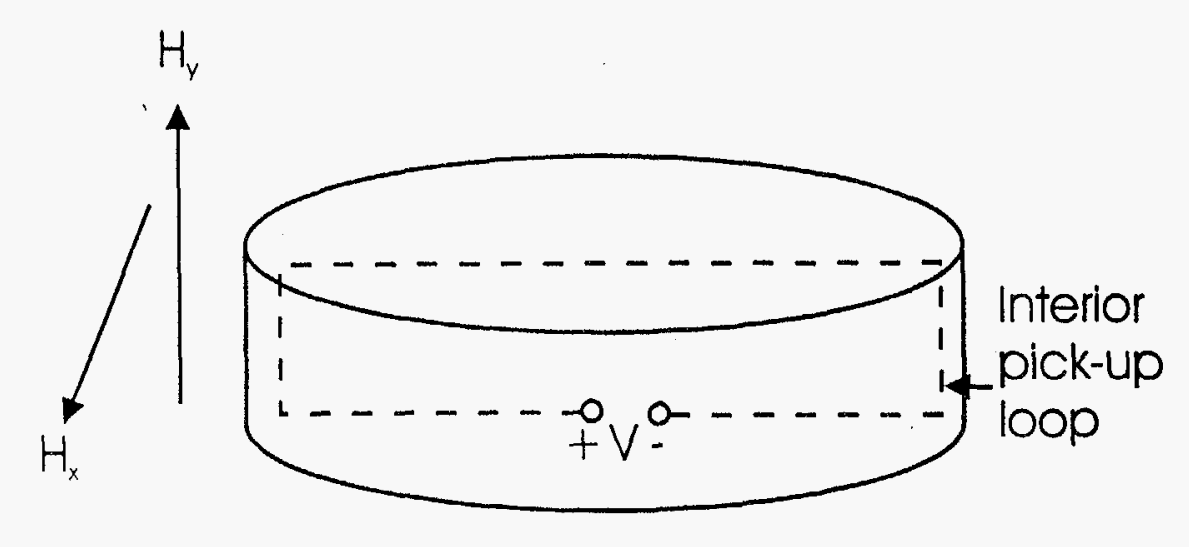

- Uniform field excitation very instructive (valid if, say, $\rho_{0} \approx b_{0} \approx 14.5 \mathrm{~mm}$, field amplitude $H_{0} \approx \frac{I_{0}}{2 \pi \rho_{0}} \approx 2.2 \mathrm{MA} / \mathrm{m}$ ) 


\section{Uniform Field Penetration}

- Step excitation $\mathrm{H}=\mathrm{H}_{0} \mathrm{u}(\mathrm{t})$

- Analysis of canonical shapes (planes, cylinders, spheres) [Lee] yields peak value

$$
\frac{\partial}{\partial \mathrm{t}} \mathrm{H}_{\text {in }}^{\text {step }} \approx 0.66 \mathrm{H}_{0} /\left(\xi \tau_{\mathrm{d}}\right)
$$

with rise time $\tau_{d} / 4$ and fall time $\xi \tau_{d}$ where $\xi \approx \frac{\mu_{0}}{\mu} \frac{V}{S_{\Delta}} \approx 1.55$ (interior volume divided by volume of enclosure walls) and diffusion time constant $\tau_{\mathrm{d}}=\mu \sigma \Delta^{2} \approx 1.8 \mu \mathrm{s}$ stainless steel $32.7 \mu \mathrm{s}$ commercial aluminum 


\section{Uniform Field Penetration (continued)}

- Results using $\mathrm{H}_{0} \approx 2.2 \mathrm{MA} / \mathrm{m}$ are

$$
\begin{aligned}
\frac{\partial}{\partial t} H_{\text {in }}^{\text {step }} \approx 520 \mathrm{kA} / \mathrm{m} / \mu \mathrm{s} & \text { stainless steel } \\
29 \mathrm{kA} / \mathrm{m} / \mu \mathrm{s} & \text { aluminum }
\end{aligned}
$$

- Using $A \approx 1.1 \mathrm{~cm}^{2}$

$$
\begin{aligned}
& \mathbf{V} \approx A \mu_{0} \frac{\partial}{\partial t} H_{i n}^{\text {step }} \approx 72 \text { volts stainless steel } \\
& 4 \text { volts aluminum }
\end{aligned}
$$




\section{Deviations of Actual Enclosure from Canonical Geometry}

- Corner Enhancement [Kaden]

- Vicinity of corner 2-4 times nominal value of $\frac{\partial}{\partial t} H_{\text {in }}^{\text {step }}$

- Circuit removed by $>1 \mathrm{~mm}$

- Transformer windings not effectively linked

- Foil Penetration

- Impedance loaded aperture theory [Lee] open aperture (stainless steel can ignore foil)

- Add images to give line dipole $\left(\mathrm{H}_{0}=2.2 \mathrm{MA} / \mathrm{m}\right)$
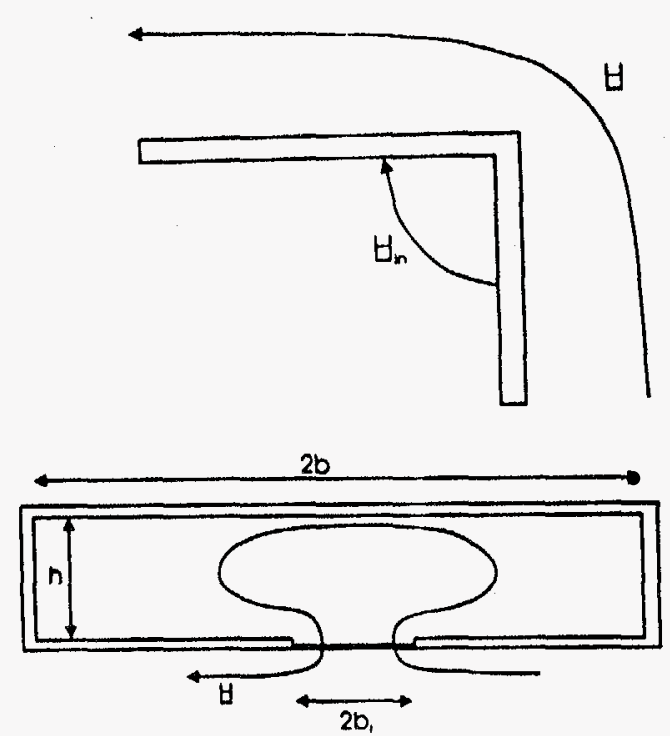

$$
\begin{aligned}
\frac{\partial}{\partial \mathrm{t}} \mathrm{H}_{\text {in }} \approx \frac{2 \mathrm{~b}_{\mathrm{f}}^{3}}{3 \pi \mathrm{h} \rho^{2}} \frac{\beta}{4} H_{0} & \approx 200 \mathrm{kA} / \mathrm{m} / \mu \mathrm{s}\left(\rho \approx 2 \mathrm{~b}_{\mathrm{f}} \approx 7 \mathrm{~mm}\right) \text { stainless steel } \\
& \approx 85 \mathrm{kA} / \mathrm{m} / \mu \mathrm{s} \quad \text { aluminum }
\end{aligned}
$$

- Stainless steel results small

- Aluminum results larger than direct diffusion (for $\mathrm{H}_{0}=2.2 \mathrm{MA} / \mathrm{m}$ )

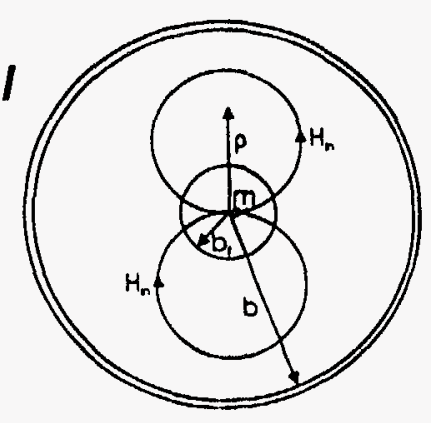

- Line source excitation (foil remains fixed while diffusive penetration increases) 


\section{D FEM Geometry}

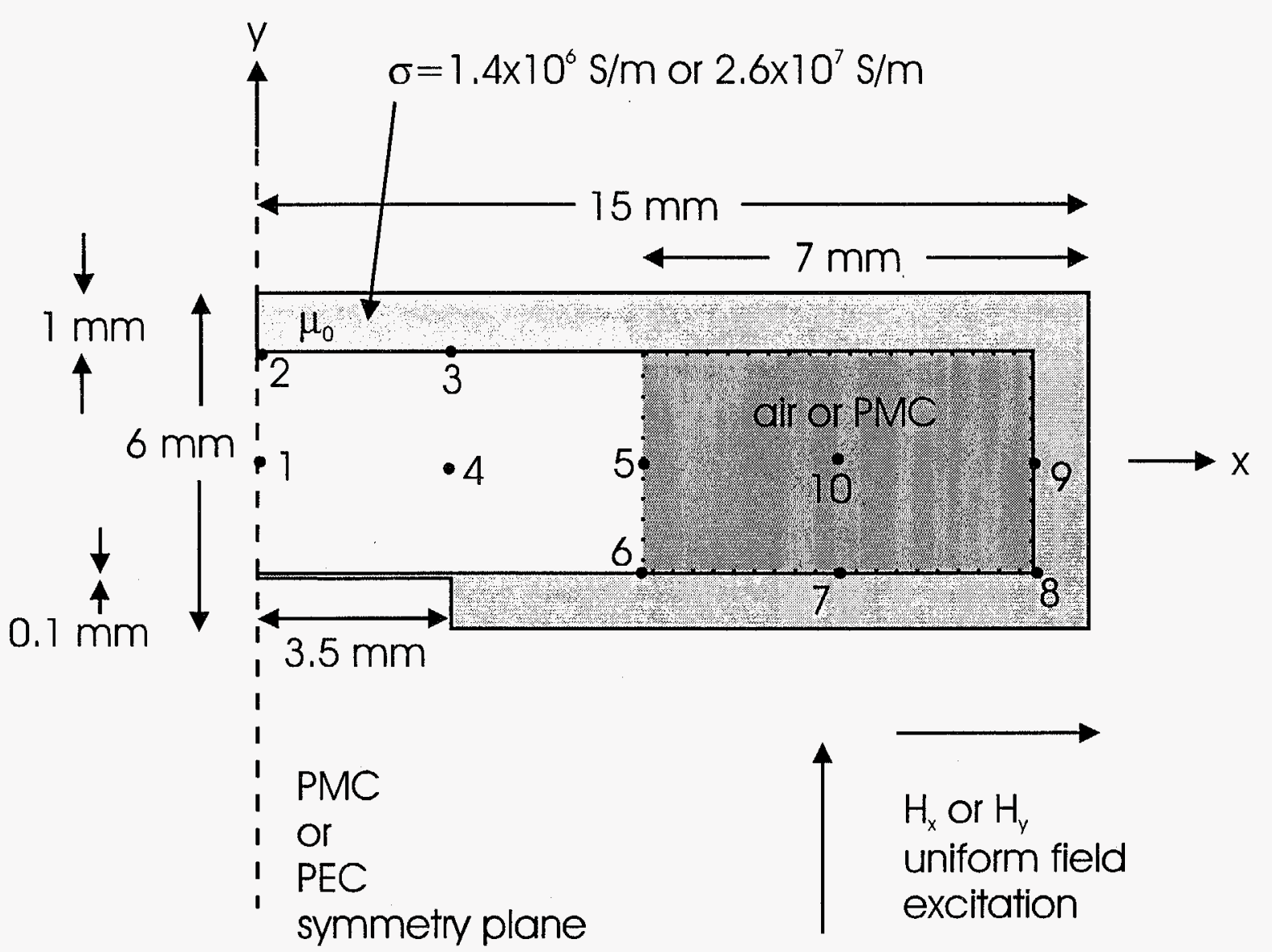

Uniform field excitation (saturated transformer or air core) stainless steel 


\section{Penetrant Magnetic Field Derivative \\ Stainless Steel Enclosure, Air Core, $\mathrm{H}^{\text {inc }}=\mathrm{H}_{\mathrm{x}}=2.2 \mathrm{MA} / \mathrm{m}$}

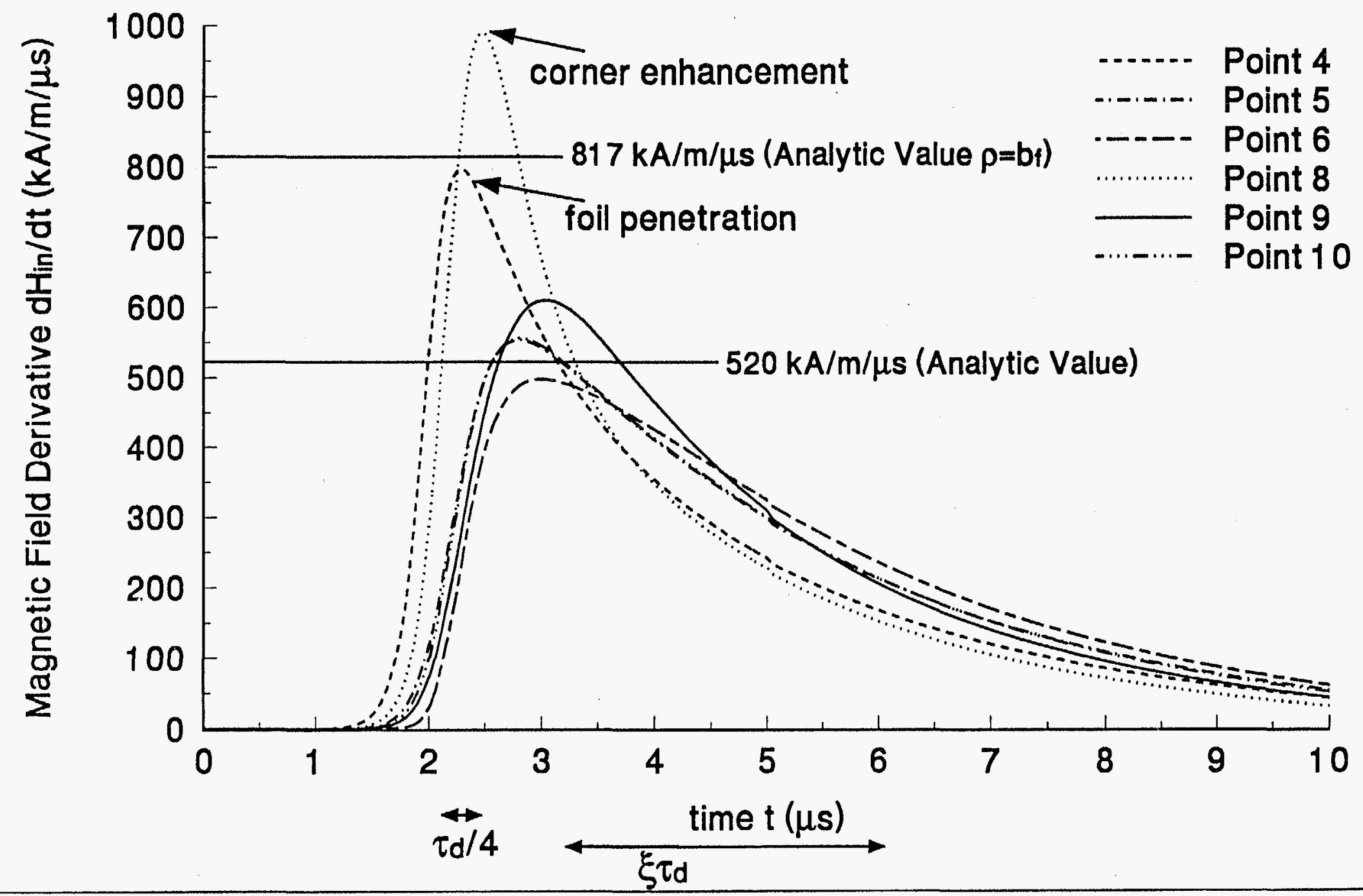




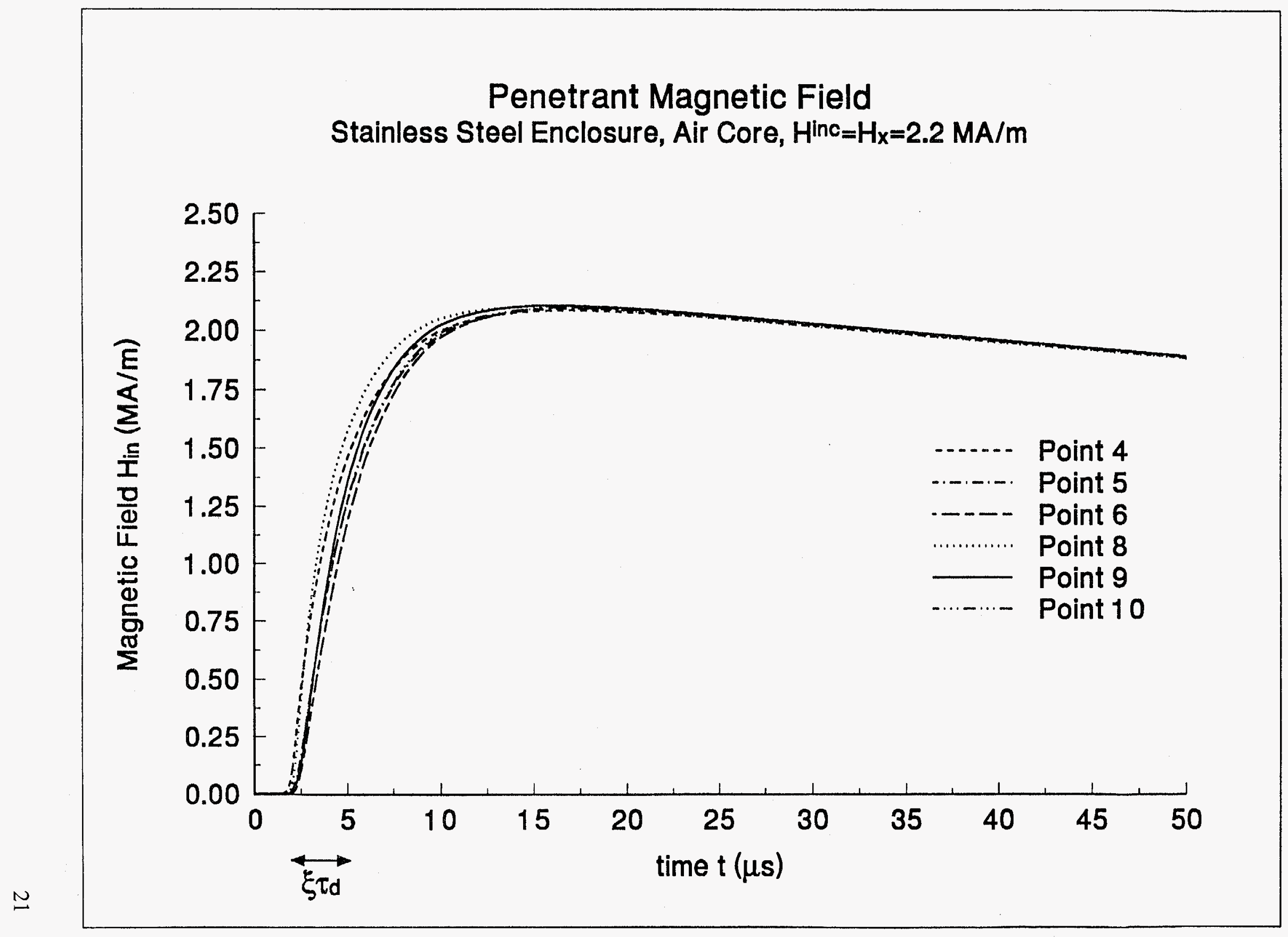

Figure 0n. 
- Theoretical worst case (step excitation, $\tau_{d} / 4$ rise time of voltage)

$$
\mathrm{V} \approx \frac{4 \ell l_{0}}{\pi e \sigma \Delta^{2}}(0.54) \approx \begin{array}{r}
976 \text { volts (stainless steel) } \\
53 \text { volts (aluminum) }
\end{array}
$$

- Practical worst case $\left(\rho_{0}=3 \mathrm{~mm}\right)$ (thin sheet impedance model)

$$
\mathrm{V} \approx \frac{\mathrm{I}_{0} l}{\pi \sigma \Delta \rho_{0}}
$$$$
\approx 409 \text { volts (stainless steel) }
$$
22 volts (aluminum)

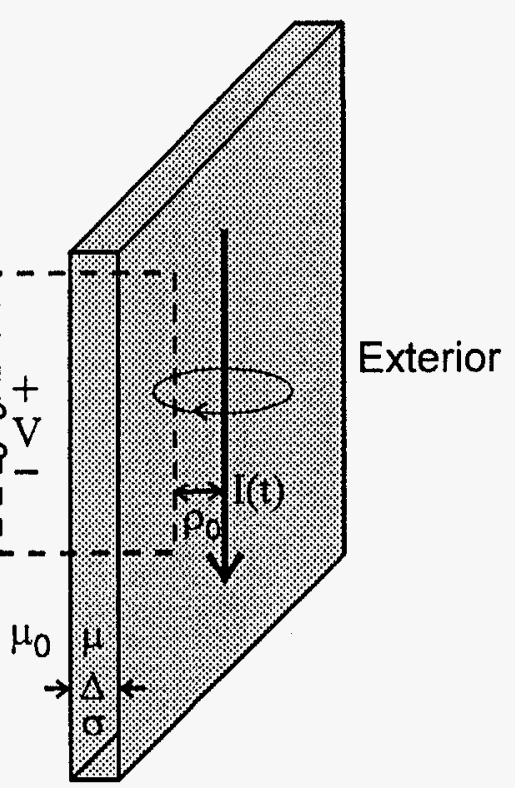

- Interesting that if we multiply previous uniform field

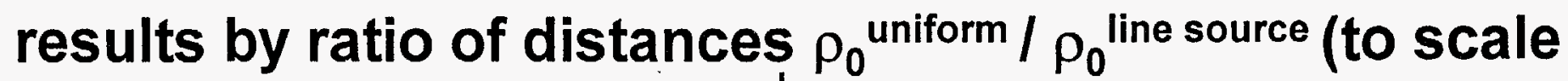
up incident field level $\mathrm{H}_{0} \approx \frac{\mathrm{I}_{0}}{2 \pi \rho_{0}}$ ) they are approximately the same as these voltages 


\section{Direct Transformer Coupling}

- Penetrant field is far above saturation level of ferrite

$$
H_{\text {in }} \approx H_{0} \quad\left(t=\xi \tau_{d}\right) \quad \text { particularly for stainless steel }
$$

(2.2 MA/m $\left.\approx \mathrm{H}_{0}\right) \quad \mu_{0} \mathrm{H}_{0} \approx 2.8 \mathrm{~T} \gg \mathrm{B}_{\mathrm{S}} \approx 0.4 \mathrm{~T}$ saturation induction

- Open circuit voltage of secondary with air core

$$
\mathrm{V} \approx \mathrm{N}_{\mathrm{s}} \pi \mathrm{b}_{\mathrm{w}}^{2} \mu_{0} \frac{\partial}{\partial \mathrm{t}} \mathrm{H}_{\text {in }}^{\text {step } \approx} \begin{aligned}
& 4.3 \mathrm{kV} \text { stainless steel } \\
& 242 \text { volts aluminum }
\end{aligned}
$$

where $N_{\text {sec }}=400$ and $b_{w} \approx 2.3 \mathrm{~mm}$ is the average secondary winding radius

- Worst-case nearby wire excitation

Thin sheet impedance solution $\rho_{0} \approx 4 \mathrm{~mm}$

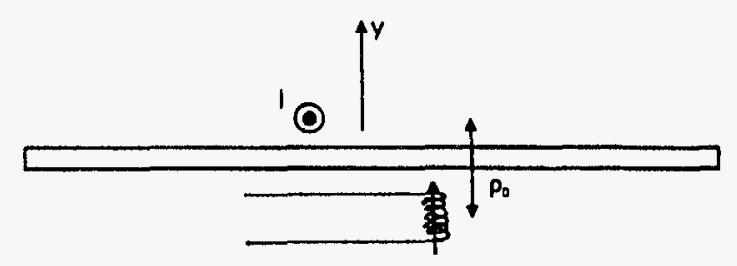

Peak $\frac{\partial}{\partial \mathrm{t}} \mathrm{H}_{\mathrm{y}} \approx \frac{3 \sqrt{3}}{8 \pi} \frac{\mathrm{I}_{0}}{\mu_{0} \sigma \Delta \rho_{0}^{2}} \approx 1500 \mathrm{kA} / \mathrm{m} / \mu \mathrm{s}$ stainless steel

Nearly three times uniform field amplitude $520 \mathrm{kA} / \mathrm{m} / \mu \mathrm{s}$ 


\section{Crude Secondary Circuit}

- Assume primary shorted

- Use finite solenoid formula $-\mathrm{h}_{\mathrm{s}} \approx 2.9 \mathrm{~mm}$ coil length - for rough estimate of leakage inductance

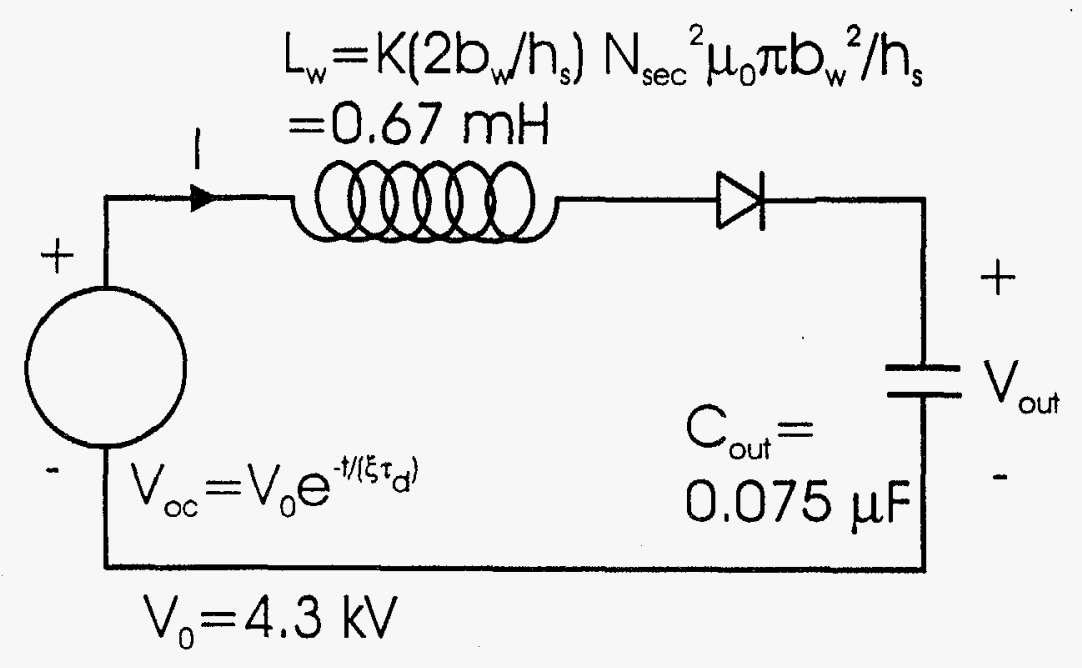

- Because oscillation period long compared with $\xi \tau_{d}$ (stainless steel)

$$
\mathrm{W}_{\text {out }} \approx \mathrm{V}_{0}^{2} \xi^{2} \tau_{\mathrm{d}}^{2} /\left(2 \mathrm{~L}_{\mathrm{w}}\right) \approx 107 \mathrm{~mJ}
$$

- Reduced but greater than threshold - for one return stroke - Incorporation of primary loads may reduce below threshold 2700:95:0027p: 16 


\section{Energy Bounds}

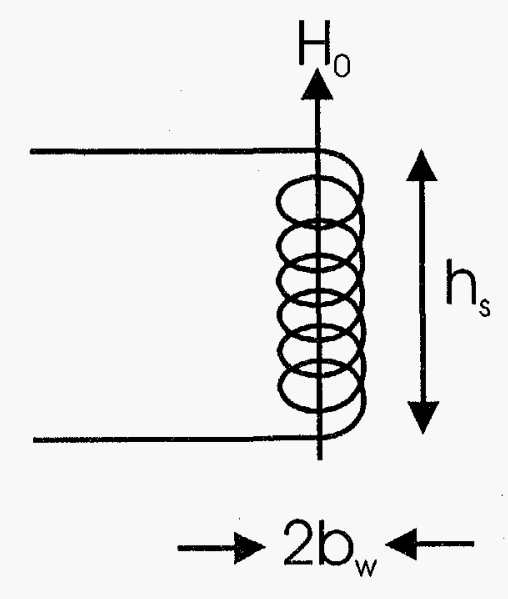

- Energy stored in solenoid assuming $\mathrm{H}_{\mathrm{in}} \approx \mathrm{H}_{0}$ and core is saturated

$$
\mathrm{W} \approx \frac{1}{2} \mu_{0} \mathrm{H}_{0}^{2}\left(\pi \mathrm{b}_{\mathrm{w}}^{2} \mathrm{~h}_{\mathrm{s}}\right) / \mathrm{K}\left(2 \mathrm{~b}_{\mathrm{w}} / \mathrm{h}_{\mathrm{s}}\right) \approx 253 \mathrm{~mJ}
$$

- Greater than threshold Of course, this incorporates nothing about circuit impedance mismatches 


\section{Outer Shield Wall}

- Consider stainless steel enclosure with outer shield wall of stainless steel of thickness $\Delta_{S}=3 \mathrm{~mm}$

- Practical worst-case of wire current gives (thin sheet impedance model)

$$
\begin{aligned}
& \mathrm{V} \approx \frac{2 \mathrm{I}_{0} \ell}{\pi \sigma \Delta}\left(\frac{1}{\rho_{0}^{\text {(close })}}-\frac{1}{\rho_{0}^{(\mathrm{far})}}\right) \\
& \approx 34 \text { volts }
\end{aligned}
$$

- Transformer coupling with worst-case wire current

$$
\begin{aligned}
& \frac{\partial}{\partial \mathrm{t}} \mathrm{H}_{\mathrm{y}} \approx \frac{3 \sqrt{3}}{8 \pi} \frac{\mathrm{I}_{0}}{\mu_{0} \sigma \Delta_{\text {tot }} \rho_{0}^{2}} \approx 120 \mathrm{kA} / \mathrm{m} / \mu \mathrm{s} \\
& \mathrm{V} \approx \mathrm{N}_{\mathrm{sec}} \pi \mathrm{b}_{\mathrm{w}}^{2} \mu_{0} \frac{\partial}{\partial \mathrm{t}} \mathrm{H}_{\mathrm{y}} \approx 1 \mathrm{kV}
\end{aligned}
$$

$0^{(1+1)}$
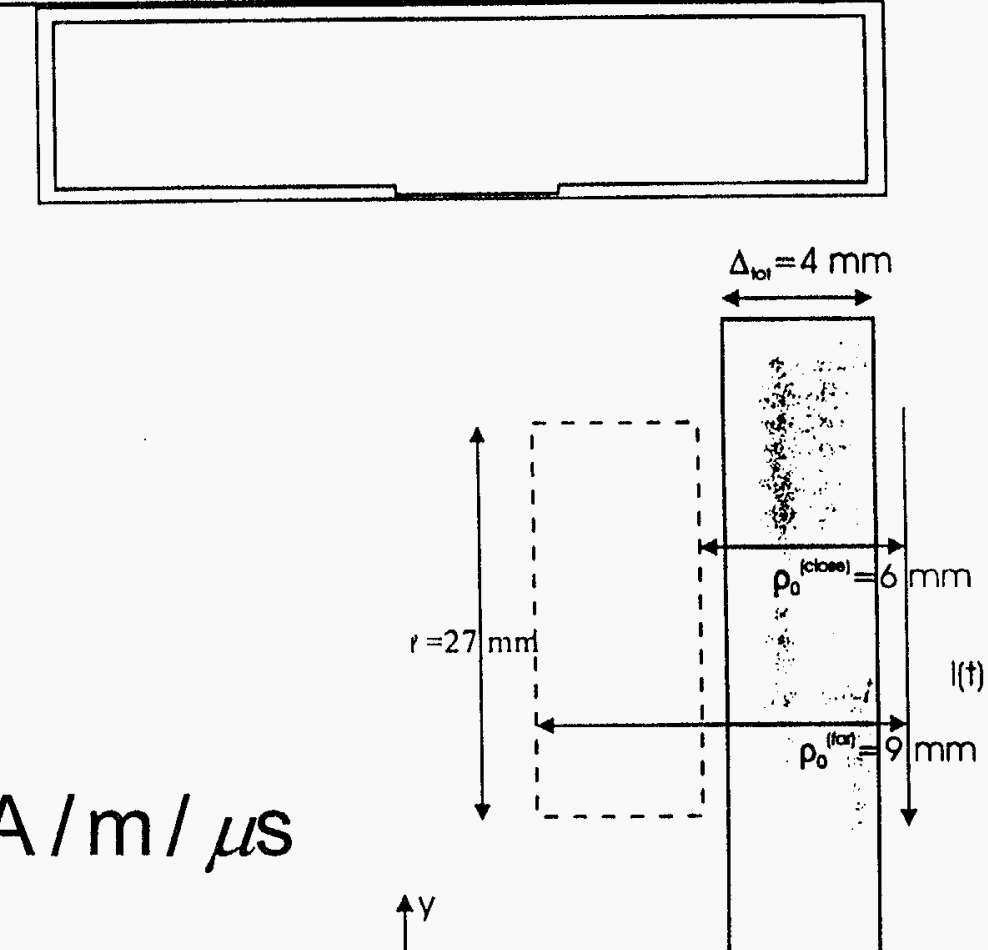

- Output open circuit voltage just above threshold 


\section{Power Line Threat}

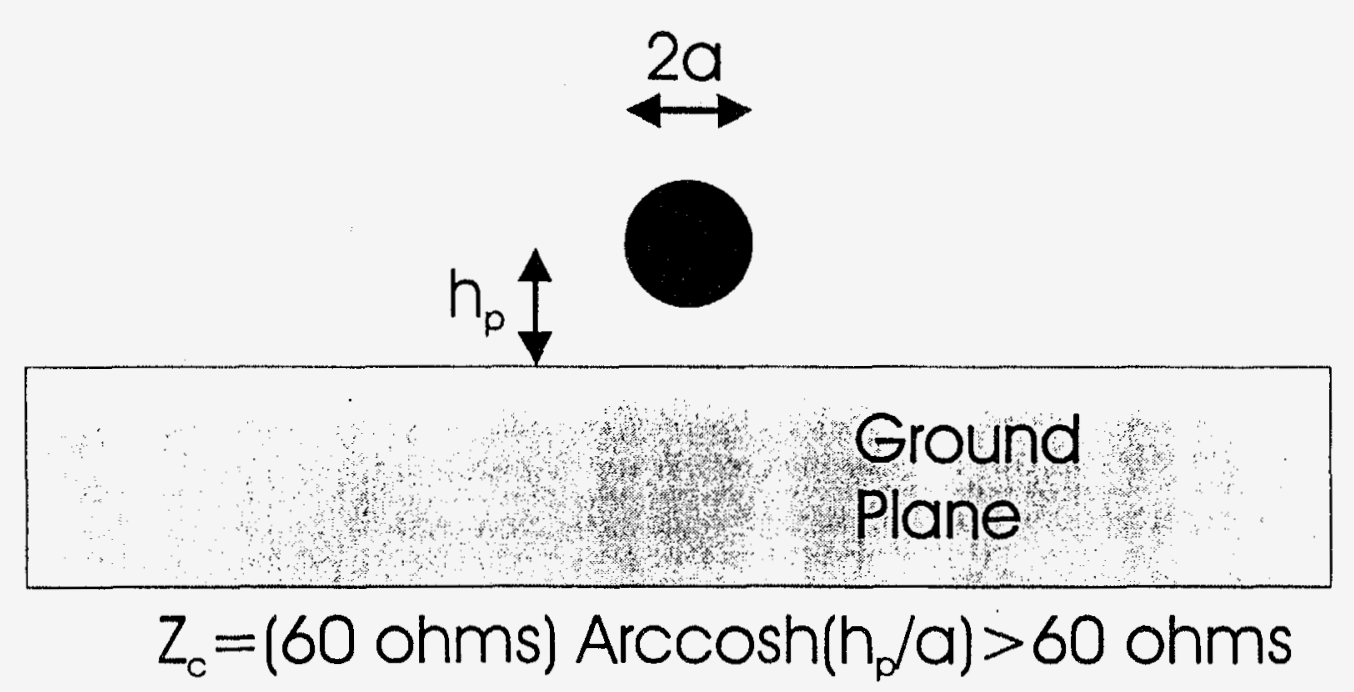

- High voltage lines $(750 \mathrm{kV})$ are expected to deliver surge currents of tens of kilo amperes

- The threat posed is thus thought to be less severe than lightning 
- Aluminum enclosure

- Uniform field drive $\mathrm{H}_{\text {in }} \approx(0.5-0.7) \mathrm{H}_{0}(50-\mu \mathrm{s}$ rise $)$ for $\mathrm{H}_{0} \approx 2.2 \mathrm{MA} / \mathrm{m}$

$$
\frac{\partial}{\partial \mathrm{t}} \mathrm{H}_{\text {in }} \approx 29 \mathrm{kA} / \mathrm{m} / \mu \mathrm{s} \quad(8-\mu \mathrm{s} \text { rise, } 50-\mu \mathrm{s} \text { fall) }
$$

Loop voltage $V \approx 4$ volts $\left(A \approx 1.1 \mathrm{~cm}^{2}\right)$ (near foil greater voltage by factor of 2$)$

Transformer $\quad V \approx 242$ volts saturated air core

- Wire current drive

Loop voltage $V \approx 22$ volts $\left(\rho_{0} \approx 3 \mathrm{~mm}, l \approx 27 \mathrm{~mm}\right)$

Transformer $V \approx 661$ volts $\left(\rho_{0} \approx 4 \mathrm{~mm}, N_{\text {sec }}=400, b_{W} \approx 2.3 \mathrm{~mm}\right)$

- All below threshold $V \approx 700$ volts 


\section{Summary (continued)}

- Stainless steel enclosure

- Uniform field drive $\mathrm{H}_{\text {in }} \approx(0.9-1) \mathrm{H}_{0}(3-\mu \mathrm{s}$ rise $)$ for $\mathrm{H}_{0} \approx 2.2 \mathrm{MA} / \mathrm{m}$

$$
\begin{aligned}
& \frac{\partial}{\partial \mathrm{t}} \mathrm{H}_{\text {in }} \approx 520 \mathrm{kA} / \mathrm{m} / \mu \mathrm{s} \quad(0.5-\mu \mathrm{s} \text { rise, } 3-\mu \mathrm{s} \text { fall }) \\
& \text { Loop voltage } V \approx 72 \text { volts }\left(A \approx 1.1 \mathrm{~cm}^{2}\right) \text { (near foil } \\
& \text { slightly greater) } \\
& \text { Transformer } \quad \mathrm{V} \approx 4.3 \mathrm{kV} \text { saturated air core } \\
& W \approx 107 \mathrm{~mJ} / \text { return stroke primary shorted }
\end{aligned}
$$

- Wire current drive

Loop voltage $V \approx 409$ volts $\left(\rho_{0} \approx 3 \mathrm{~mm}, l \approx 27 \mathrm{~mm}\right.$ )

Transformer $V \approx 11 \mathrm{kV}\left(\rho_{0} \approx 4 \mathrm{~mm}, \mathrm{~N}_{\mathrm{sec}}=400, \mathrm{~b}_{\mathrm{W}} \approx 2.3 \mathrm{~mm}\right)$

- Above threshold $V \approx 700$ volts, $W_{\text {out }}=18.4 \mathrm{~mJ}$ 
- Stainless steel enclosure with additional 3-mm stainless shield wall

- Wire current drive

Loop voltage $V \approx 34$ volts

Transformer $\quad V \approx 1 \mathrm{kV}\left(\rho_{0} \approx 7 \mathrm{~mm}, \mathrm{~N}_{\mathrm{sec}}=400, \mathrm{~b}_{\mathrm{w}} \approx 2.3 \mathrm{~mm}\right)$

- Above but near threshold $V \approx 700$ volts

- Overestimates (likely that true answers below threshold) 


\section{Conclusions}

- Worst-case penetrant field and field time derivatives provided

- Open circuit voltages below threshold for aluminum enclosure - flyback transformer step-up performance should be assessed

- Open circuit voltages and energies above threshold for stainless steel

- More detailed interior circuit modeling and transformer modeling may reduce voltages and energies delivered to capacitor 
- Addition of 3-mm stainless steel outer shield wall reduces open circuit voltages in stainless steel enclosure to above but near threshold

- With built-in margin of estimates true values believed to be below threshold

- Power line threat less severe than lightning

- Simple models for penetrant fields provide drives for more detailed interior circuit models

- Understanding of the diffusive penetrations can be used to set up effective test series 


\section{Magnetic Field Diffusion Modeling of a Small Enclosed Firing System}

\section{INTRODUCTION}

Induced voltages, resulting from time changing magnetic fields, are a familiar problem in electronic circuits, and as a result, conducting barriers (shields) are often used to mitigate this effect. The conducting barriers do not prevent the diffusive penetration of low frequency magnetic field components but reduce the penetration of the high frequency magnetic field components. Thus the rate of rise is reduced and induced interior voltages are reduced. Nevertheless if the exciting field is taken to extreme levels, as in the case of the immediate vicinity of a lightning strike (or a power line cable), it is worthwhile to assess the effectiveness of the conducting barrier and thus the interior threat to circuitry and components. We assume here that the conducting barrier has no significant holes (holes for fiber optics, for example, are assumed to be exceedingly small here) and thus diffusion in the barrier walls represents the only mode of penetration (excluding a breach of the barrier, such as lightning burnthrough).

Figure 1 shows the cylindrical pill box geometry of the conducting barrier and some details of the interior circuit layout. The radius is $b_{o}=(b+\Delta) \approx 14.5 \mathrm{~mm}$, the height is $h_{o}=(h+2 \Delta) \approx 6$ $\mathrm{mm}$, and the metallic wall thickness is $\Delta \approx 1 \mathrm{~mm}$. The inner pill box dimensions are radius $b$ and height $h$. The metallic wall material may be stainless steel with an electrical conductivity $\sigma \approx 1.4 \times 10^{6} \mathrm{~S} / \mathrm{m}$ (type 304 stainless steel [1]), or commercial aluminum with a conductivity of $\sigma \approx 2.6 \times 10^{7} \mathrm{~S} / \mathrm{m}$ (type 6061 aluminum [2]). The magnetic permeability for these materials is taken to be the same as free space $\mu=\mu_{0}=4 \pi \times 10^{-7} \mathrm{H} / \mathrm{m}$. The bottom surface of the pill box has a centered cylindrical foil region of radius $b_{f} \approx 3.5 \mathrm{~mm}$ and reduced thickness $\Delta_{f} \approx 0.1 \mathrm{~mm}$.

Interior to the barrier is a disc shaped circuit board that is parallel to the top and bottom surfaces of the pill box and has a central hole cutout of the same radial size as the reduced thickness bottom wall of the barrier. Circuit traces capable of magnetic field pickup exist on the circuit board. The circuit board also has another circular cutout of about the same radius with outer point adjacent to the cylindrical wall of the barrier. This hole contains a pot-core flyback transformer. The transformer with its many windings is a component for which direct magnetic coupling may be of concern. The output of the transformer is coupled by means of a diode to a $C_{\text {out }}=0.075$ $\mu \mathrm{F}$ capacitor with $R \approx 100 \mathrm{M} \Omega$ bleed resistor. The threshold charge voltage of the capacitor is $V_{\text {out }} \approx 700$ volts which corresponds to a charge energy of $W_{\text {out }}=\frac{1}{2} C_{\text {out }} V_{\text {out }}^{2} \approx 18.4 \mathrm{~mJ}$. Estimated bounds on induced voltage and energy can therefore be compared to these levels to assess whether magnetic field threats are of any concern (and hence warrant further investigation). The no-go levels are $V_{\text {out }}=500$ volts, $W_{\text {out }}=9.4 \mathrm{~mJ}$, which are also important for comparison in certain safety studies.

The worst case magnetic field threat (to produce interior magnetic fields) appears to be that of a thin insulated wire carrying the lightning current (or power line current) running adjacent to the exterior barrier wall (only the top barrier wall will be considered) shown in Figure 2. The case of the pill box barrier immersed in a uniform exterior field will also be treated (which is a reasonable approximation if, say, the lightning current is at least a pill box radius $b_{0}$ away from the barrier).

The philosophy used in the analysis is to first consider the penetration of the pill box if the wall thickness is uniform at $\Delta$ with uniform field excitation. The penetrant field enhancements at the corners of the pill box are also discussed. Next, the central foil penetration is considered. The worst case of nearby wire current excitation is then addressed. Magnetic field coupling to wire loops and directly to the transformer are both considered. Induced voltages are compared with the threshold. The nonlinear behavior of the transformer is approximately addressed. A two dimensional finite 


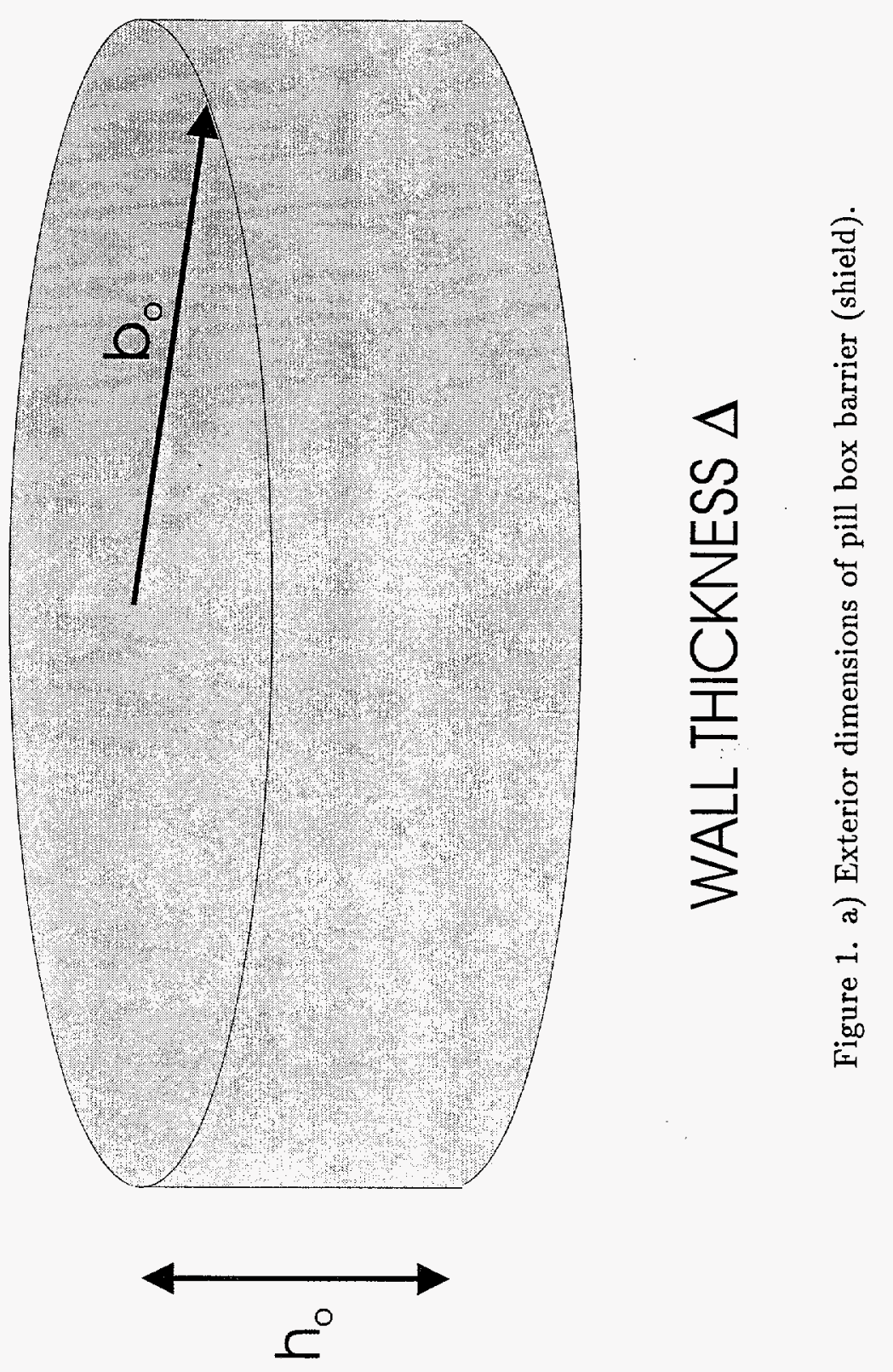




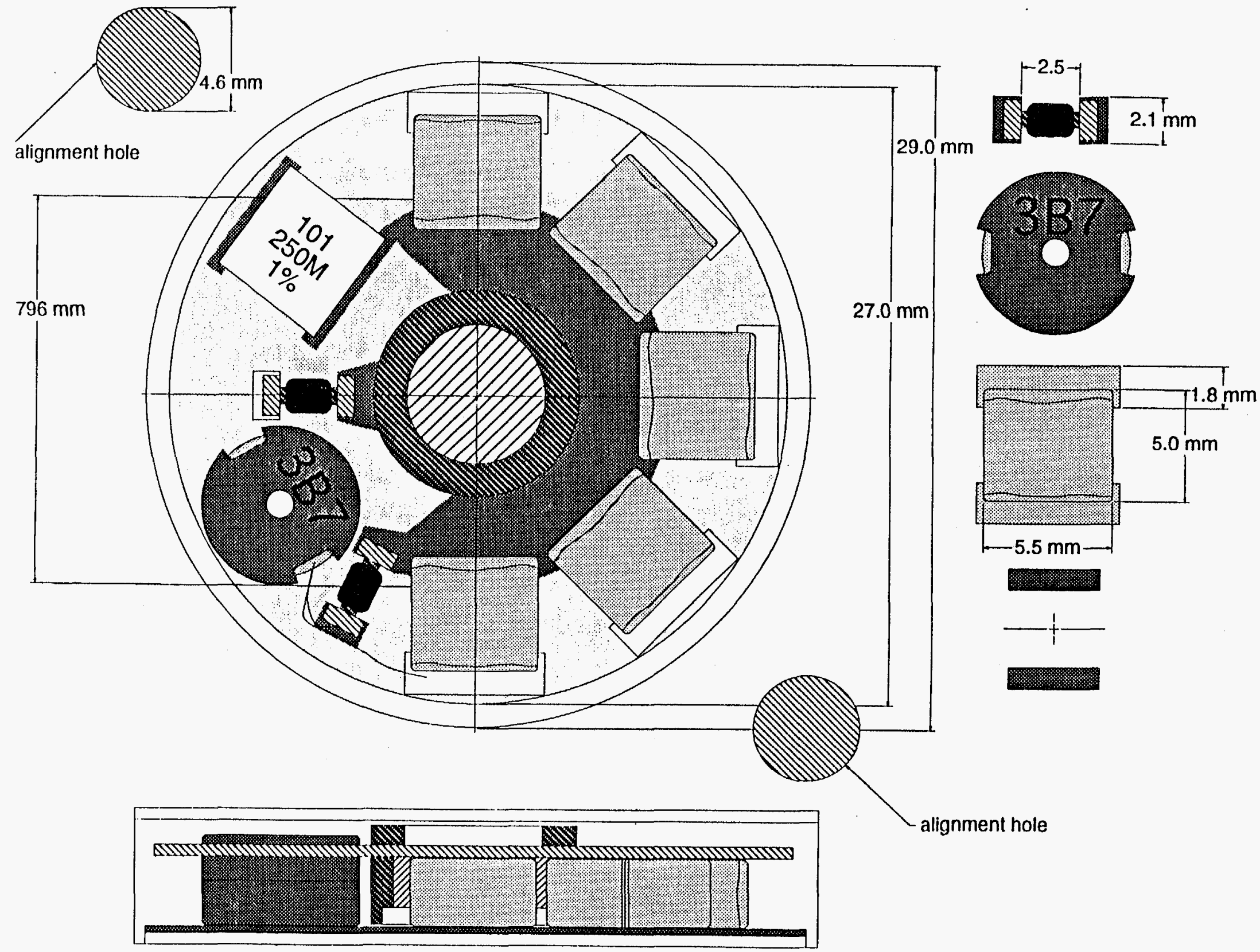

Figure 1.b) Interior layout of transformer (3B7) and circuitry. 


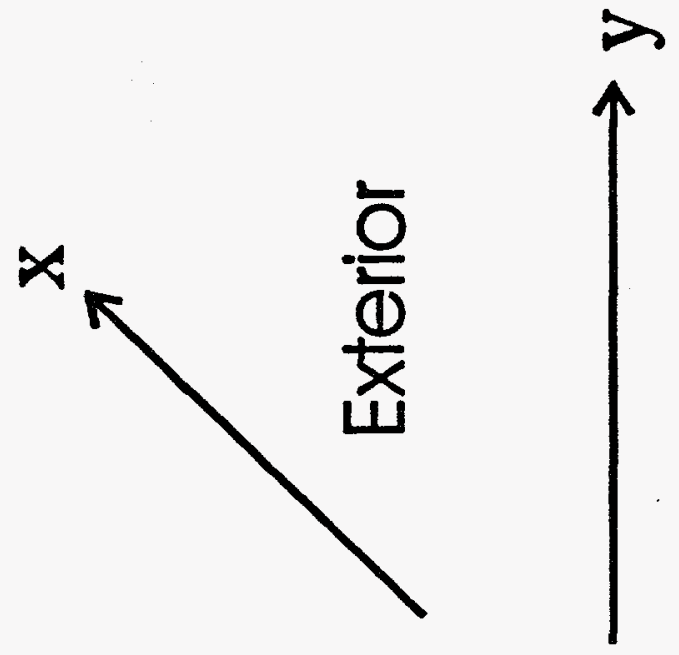

.

秀

乼

$\frac{0}{4}$

는

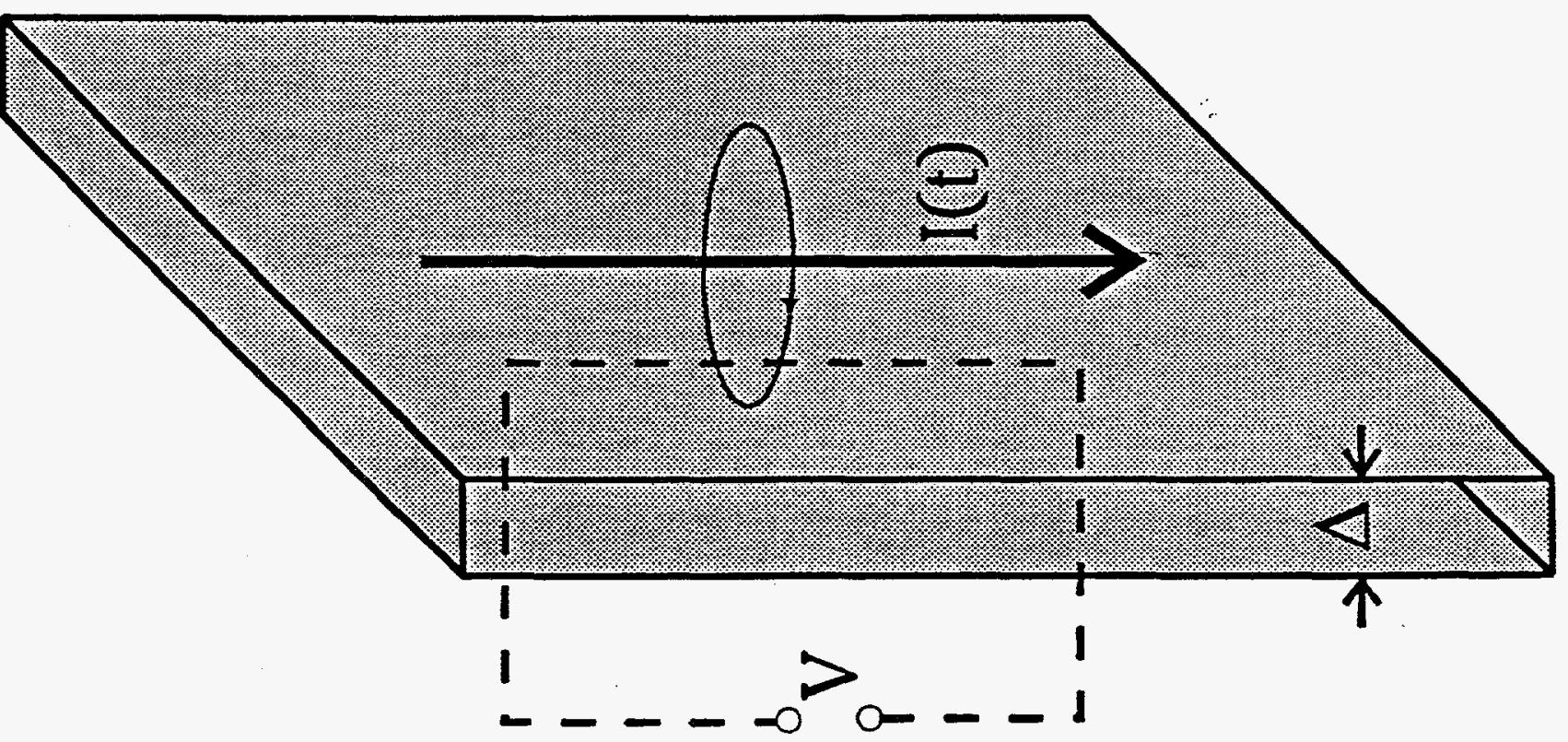

焉离

so क्ष

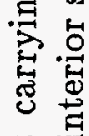

范。

언

莺䓛

苛

휵응

诘昜

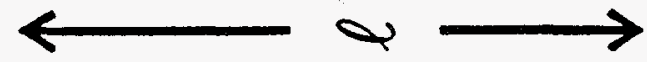

$\frac{\overline{0}}{\frac{0}{ \pm}}$ 
element numerical model of the pill box (with center foil region on the bottom of the pill box) is discussed and compared with previous analytical estimates. Bounds on energy coupling to interior loops are considered and compared with the charge energy threshold. The effect of an additional conducting barrier is also considered and shown to reduce the penetration to smaller levels. Finally power line fields are addressed.

\section{LIGHTNING MAGNETIC FIELD}

To begin we consider the form of the lightning current. Lightning consists of a leader phase, during which the lightning channel is formed, and a return stroke phase [4]. During the leader phase upward propagating streamers may form from objects on the ground and result in hundreds of amperes of current at the ground. The return strokes have large currents, up to several hundred thousand amperes with up to several hundred microsecond durations. Between return strokes smaller continuing currents may flow with amplitudes up to several hundred amperes and durations of up to several seconds. The return stroke currents are the most threatening in the present problem, because the levels are so large and the fall times typically as great or greater than the barrier diffusion time. A statistical study of the frequency of the various lightning parameters was carried out [3] (other more recent compilations are also available [4], [5]). Standard practice has been to use $1 \%$ worst case parameters (worse than $99 \%$ of naturally occurring lightning) for safety studies.

Figure 3 shows statistical frequency graphs of some of these parameters from [3]. The $1 \%$ worst case parameters from these graphs, which are relevant here, are: $200 \mathrm{kA}$ peak current (first return stroke, $80 \mathrm{kA}$ subsequent return strokes), $200 \mu \mathrm{sec}$ fall to half peak, 12 return strokes per flash, $0.2 \mu \mathrm{sec}$ time to peak, $120 \mathrm{kA} / \mu \mathrm{sec}$ maximum rise rate. A double exponential fit to the peak amplitude, rise time, and fall time (for definiteness the peak to half peak fall time), is

$$
I(t)=I_{0}\left(e^{-\alpha t}-e^{-\beta t}\right) u(t)
$$

where $I_{0} \approx 200 \mathrm{kA}, \alpha=0.00347 \mu \mathrm{sec}^{-1}\left(\alpha=0.0154 \mu \mathrm{sec}^{-1}\right.$ which corresponds to a fall time of $45 \mu \mathrm{sec}$ for the $50 \%$ worst case lightning) and $\beta=47.7 \mu \mathrm{sec}^{-1}$, and $u(t)$ is the unit step function. Metallic barriers of typical thicknesses have diffusion times that are long compared to the lightning rise time (at least of subsequent return strokes) and thus an adequate waveform is the decaying exponential

$$
I(t) \approx I_{0} e^{-\alpha t} u(t)
$$

If, in addition, the fall time is long compared to the diffusion time (this provides a worst case estimate in any event) we use the approximate step function waveform

$$
I(t) \approx I_{0} u(t)
$$

The thin foil region of the shield has small enough thickness that the diffusion times associated with it are comparable to the worst case lightning current rise times. Further consideration of an appropriate waveform for the rising portion of the waveform is necessary because the maximum rise rate associated with the fit (1) is too large (this results mostly from the fact that the largest rates of rise are not generally associated with the largest peak currents). The double exponential waveform (1) is also known to have too large a rise rate at very early times $t \rightarrow 0$. A waveform that is often used instead is the inverse exponential waveform [6]

$$
I(t)=\frac{I_{0} U e^{\alpha t}}{1+e^{\beta\left(t-t_{p}\right)}}
$$




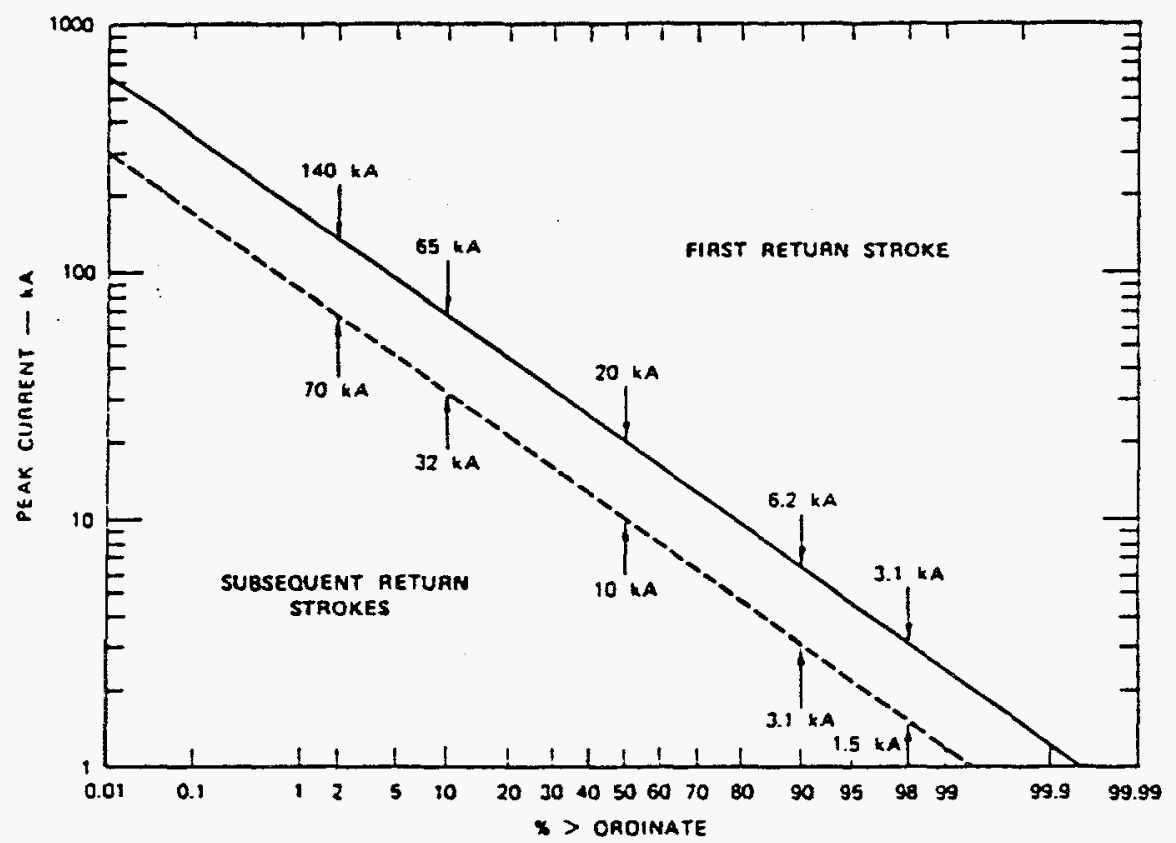

Figure 3. Lightning statistical distributions (from [3]). a) Peak current distribution.

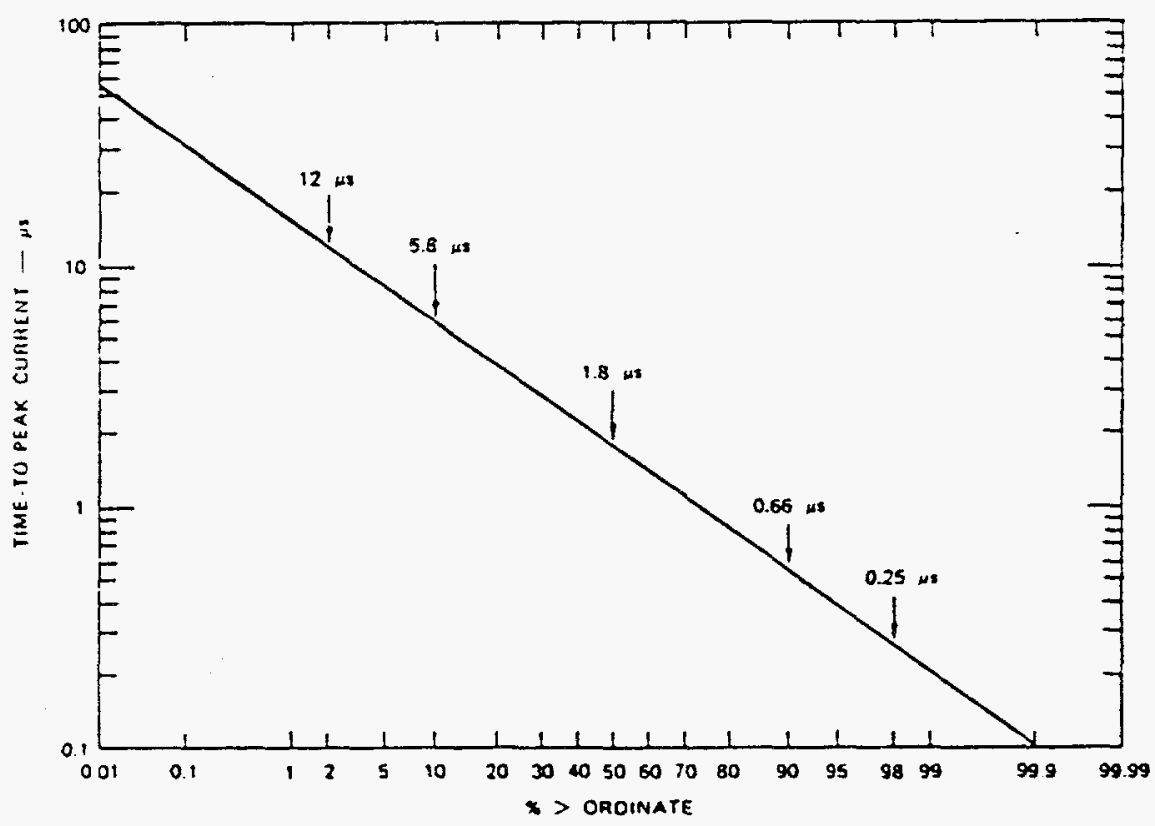

Figure 3. Lightning statistical distributions (from [3]). b) Time to peak current. 


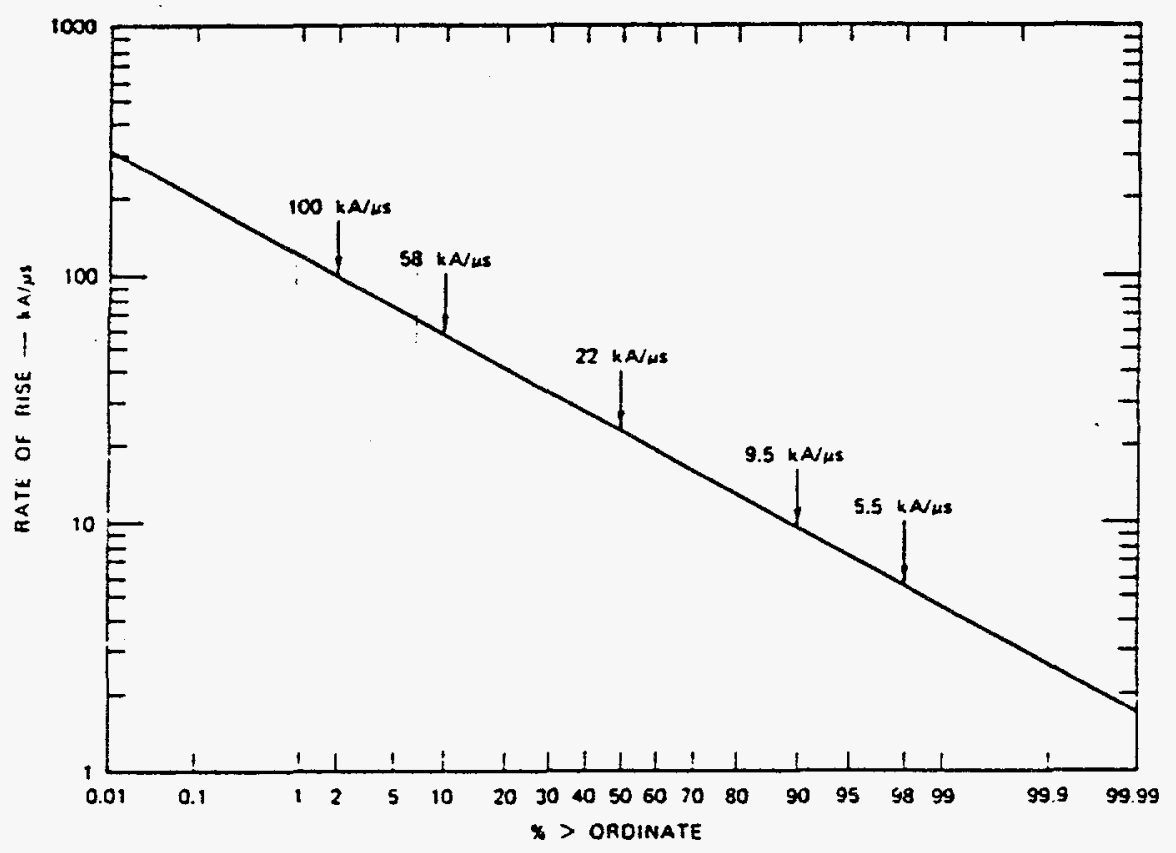

Figure 3. Lightning statistical distributions (from [3]). c) Rate of rise.

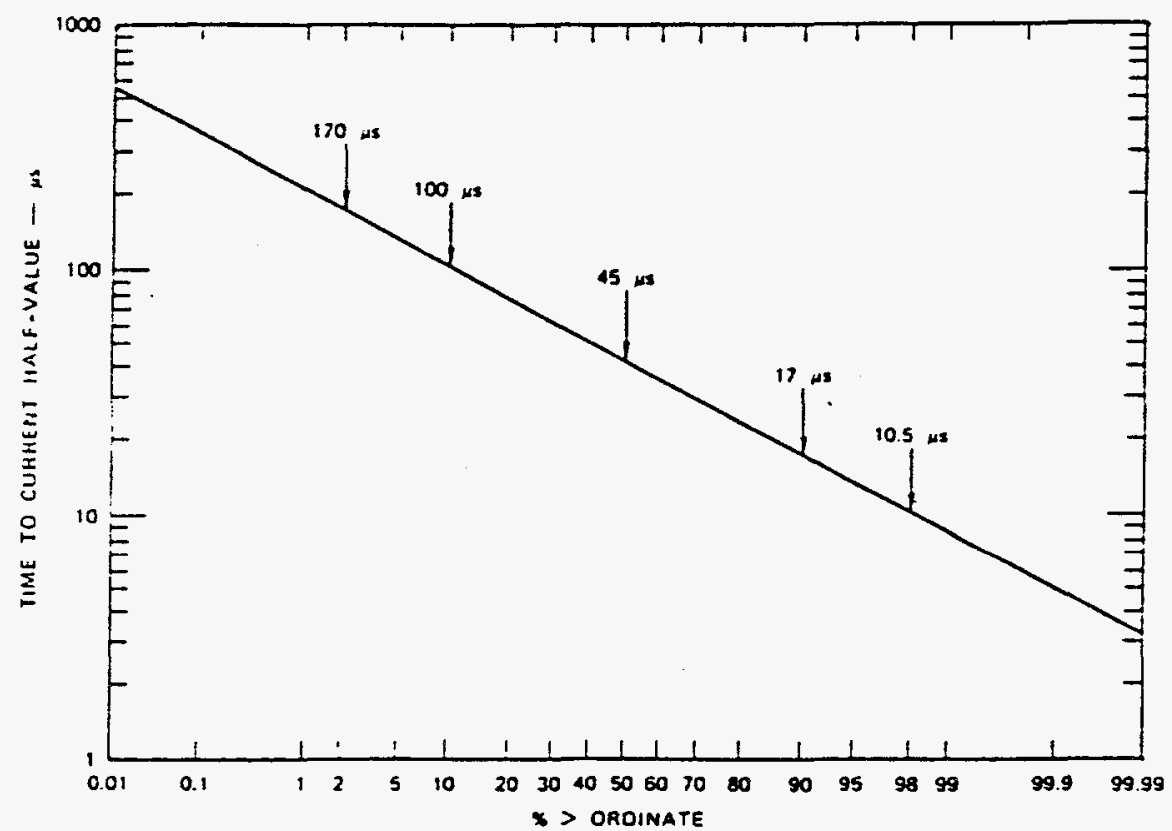

Figure 3. Lightning statistical distributions (from [3]). d) Time to half peak value of current. 


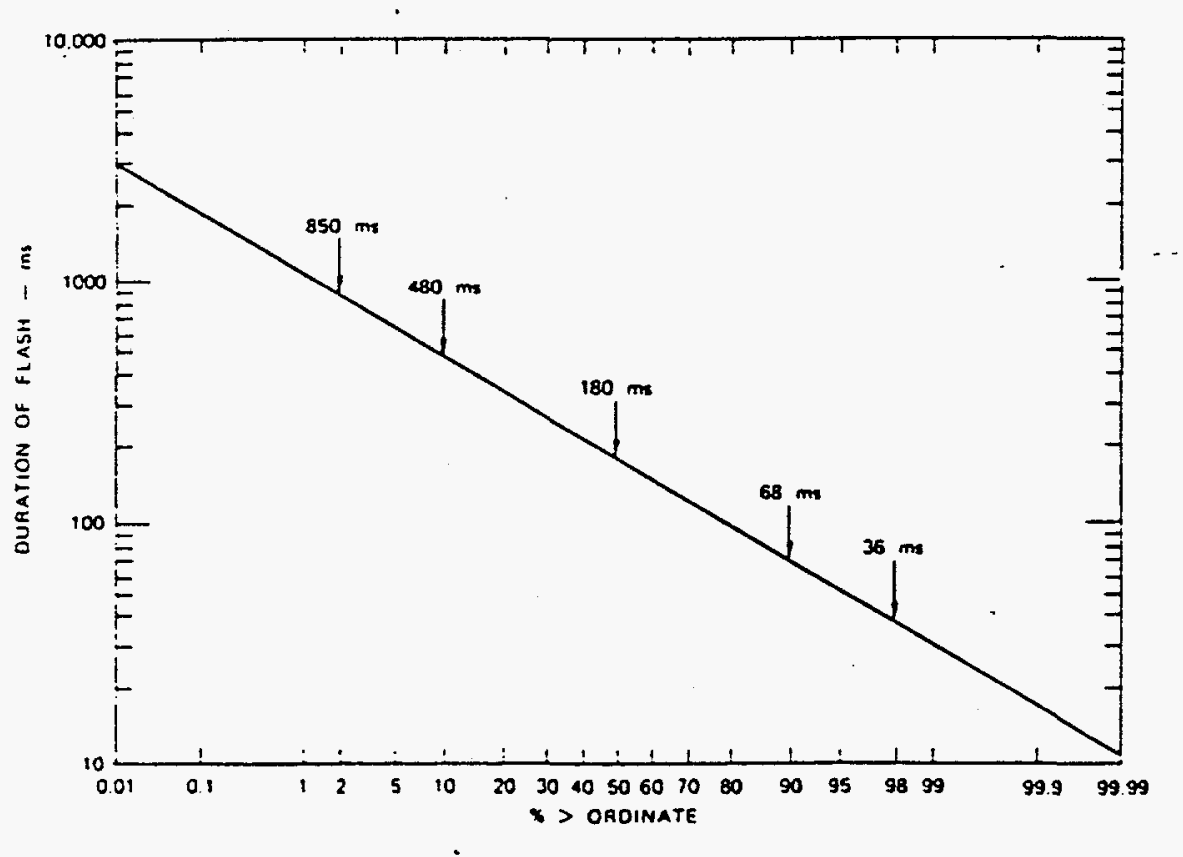

Figure 3. Lightning statistical distributions (from [3]). e) Duration of flashes (including several return strokes and between stroke intervals).

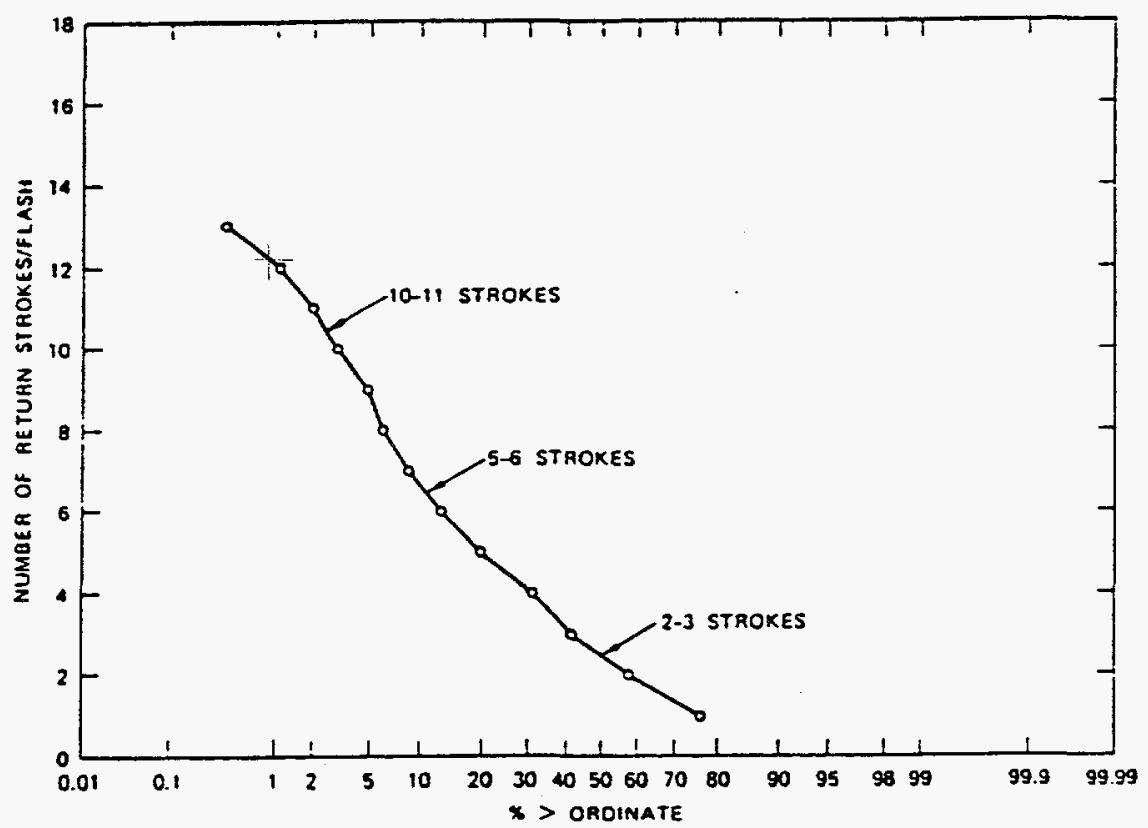

Figure 3. Lightning statistical distributions (from [3]). f) Number of return strokes per flash. 


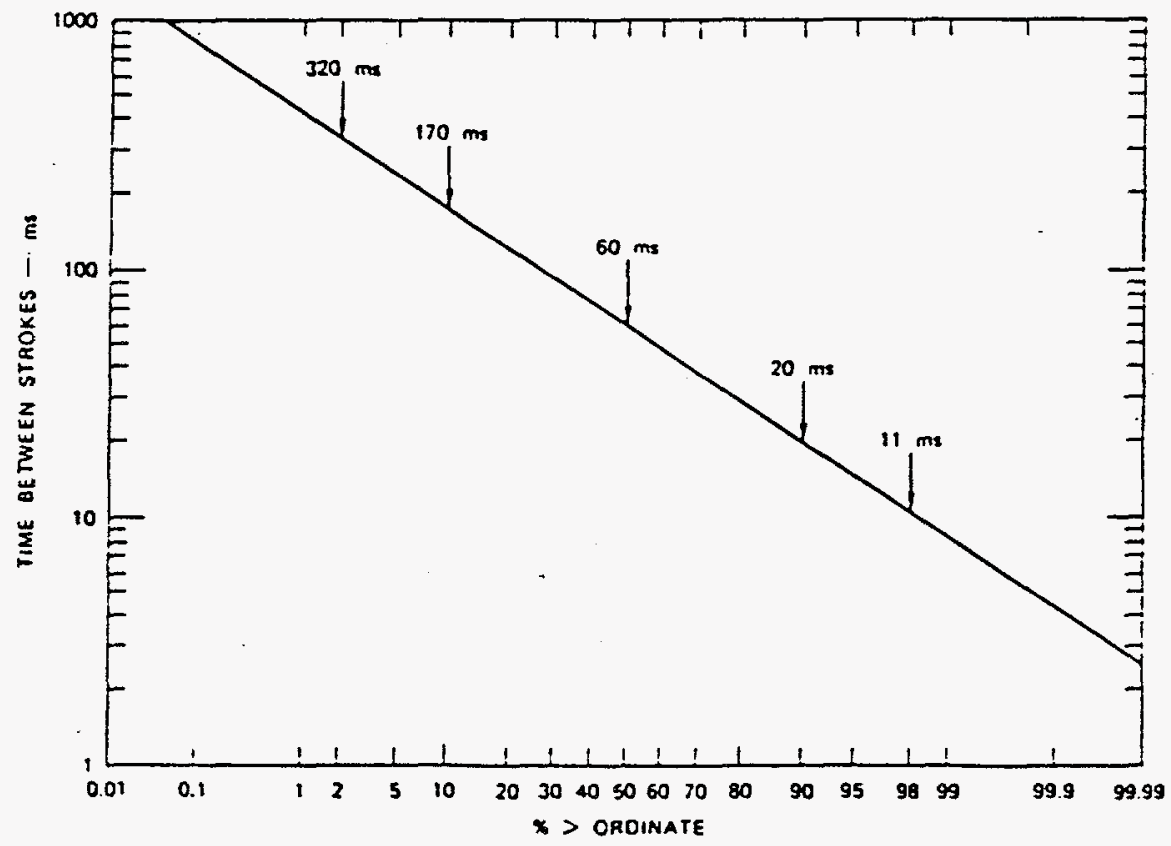

Figure 3. Lightning statistical distributions (from [3]). g) Time interval between return strokes.
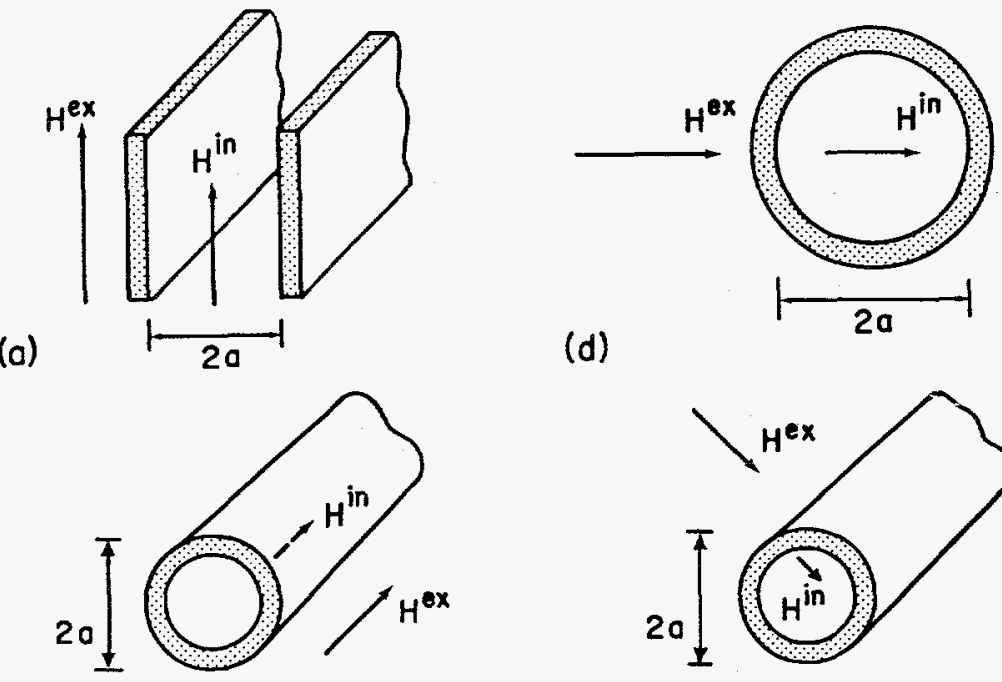

(b)

(d)

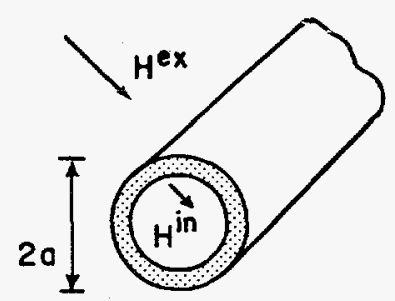

(c)

Figure 4. Canonical diffusion barriers (from [6]), including planes, cylinders, and spheres. 
where $U=\beta(\beta-\alpha)^{\alpha / \beta-1} \alpha^{-\alpha / \beta} e^{-\alpha t_{p}}$, the peak amplitude is $I_{0}$, and $t_{p}$ is a shift time (so the waveform rises from very near zero at $t=0$ ). The parameters $\alpha$ and $\beta$ are most frequently fit by using the $10 \%$ to $90 \%$ of peak rise time and the half peak fall time. However, for the study performed here, it is thought that the peak rise rate, the peak amplitude, and the half peak fall time are the most critical parameters. We therefore use the peak rise rate value $[5] \frac{\partial}{\partial t} I=400$ $\mathrm{kA} / \mu \mathrm{sec}$, the peak value $I_{0}=200 \mathrm{kA}$, and we will take the fall time from peak to half peak value to be $t_{h}=200 \mu \mathrm{sec}$ (longer fall times are also reported [5], which would allow the penetrant field of the aluminum pill box to very nearly achieve the same level as the exterior field). The peak rise rate of the inverse exponential waveform (4) for long fall time $\alpha \rightarrow \beta$ is given by [7]

$$
\frac{\partial I}{\partial t} \approx I_{0} \beta / 4
$$

Thus for the inverse exponential waveform we obtain $\beta \approx 8 \times 10^{6} \mathrm{sec}^{-1}$. The shift time can be taken as $t_{p}=2 \mu \mathrm{sec}$ (which makes the initial value of the waveform small at $t=0$ ). The inverse exponential waveform reaches a peak value at $t_{\text {peak }}-t_{p}=-\frac{1}{\beta} \ln \left(\frac{\beta-\alpha}{\alpha}\right)$. Assuming that at time $t=$ $t_{h}+t_{\text {peak }}$, we can drop unity in the denominator of $(4)$, gives $I\left(t_{h}+t_{\text {peak }}\right) / I_{0}=1 / 2 \sim(\beta / \alpha) e^{(\alpha-\beta) t_{h}}$. Using the approximation $1 / 2 \approx e^{(\alpha-\beta) t_{h}}$ gives the value $\alpha \approx 7.99653 \times 10^{6} \mathrm{sec}^{-1}$. The $10 \%$ to $90 \%$ of peak rise time of the waveform (4), with these parameters, is $0.55 \mu \mathrm{sec}$.

Assuming the current resides on a thin wire gives a magnetic field at a radius $\rho$ from the wire

$$
H(t)=\frac{I(t)}{2 \pi \rho}
$$

Corresponding to the current amplitude $I_{0}$ the exterior field amplitude is denoted as $H_{0}=I_{0} /(2 \pi \rho)$. The next section deals with the case where the pill box barrier is immersed in a uniform field. Taking the radius distance to be the smallest that a uniform field is a reasonable approximation $\rho \approx b_{o} \approx 14.5 \mathrm{~mm}$ gives a field amplitude

$$
H_{0} \approx 2.2 \mathrm{MA} / \mathrm{m}
$$

\section{PLANE FIELD PENETRATION}

Consider the case where the pill box is immersed in a plane magnetic field $\underline{H}$. The case where the magnetic field is a pulse of short duration (compared to the penetration time of the pill box) the exterior field is taken as

$$
H(t) \approx H_{0} \delta(t)
$$

where $\delta(t)$ is the Dirac delta function. The magnetic field inside the enclosure is roughly [6]

$$
\begin{gathered}
H_{i n}(t) \approx \frac{2 H_{0}}{\sqrt{\pi} \xi \tau_{d}} \sqrt{\frac{\tau_{d}}{t}} e^{-\tau_{d} /(4 t)}, t / \tau_{d} \leq 0.1 \\
H_{i n}(t) \approx \frac{H_{0}}{\xi \tau_{d}}\left[e^{-t /\left(\xi \tau_{d}\right)}-2 e^{-\pi^{2} t / \tau_{d}}+2 e^{-4 \pi^{2} t / \tau_{d}}\right], t / \tau_{d} \geq 0.1
\end{gathered}
$$

where the diffusion time is

$$
\tau_{d}=\mu \sigma \Delta^{2}
$$

(Note that the accuracy of these formulas for the present geometry are somewhat questionable since they are polarization independent and they were arrived at by considering the canonical problems 
of planes, infinite cylinders, and spheres, as show in Figure 4 from [6], but not of barriers with two finite radii of curvature or with corners. Nevertheless, comparisons with finite element results seem to indicate the general validity of these results, away from corners, as we will see later in the report.) The value of the diffusion time constant, using $\Delta \approx 1 \mathrm{~mm}$, is $\tau_{d} \approx 1.8 \mu \mathrm{sec}$ for stainless steel and $\tau_{d} \approx 32.7 \mu \mathrm{sec}$ for commercial aluminum. The parameter $\xi$ for arbitrarily shaped enclosures can be roughly taken as [6]

$$
\xi \approx \frac{\mu_{0}}{\mu} \frac{V}{S \Delta}
$$

where $V \approx h \pi b^{2} \approx 2.3 \times 10^{-6} \mathrm{~m}^{3}$ is the volume of the box and $S \approx 2 \pi b^{2}+2 \pi b h \approx 1.48 \times 10^{-3} \mathrm{~m}^{-2}$ is the surface area. Thus we find $\xi \approx 1.55$ for $\Delta \approx 1 \mathrm{~mm}$. The appropriate worst case lightning waveform is not the delta function (8) but the step function (3). The interior magnetic field can thus be found by time integration of the delta function results. However because we are primarily interested in the induced voltage in interior circuits, resulting from the penetrant magnetic field, we are primarily interested in the time derivative of the penetrant magnetic field, which is precisely the delta function response. Therefore we can write

$$
\frac{\partial}{\partial t} H_{i n}^{\text {step }}(t) \approx H_{\text {in }}^{\text {delta }}(t)
$$

where $H_{i n}^{\text {delta }}$ is given by (9). The peak of the result (9) for this value of $\xi$ is roughly (using linear interpolation of $\xi=1$ and $\xi=3$ results) [6]

$$
\xi \tau_{d} H_{i n}^{\text {delta }} / H_{0} \approx 0.66
$$

at time

$$
t / \tau_{d} \approx 0.33
$$

Using the amplitude value (7) gives the peak value

$$
\frac{\partial}{\partial t} H_{i n}^{\text {step }} \approx \begin{gathered}
5.2 \times 10^{11} \mathrm{~A} / \mathrm{m} / \mathrm{sec} \text { (stainless steel) } \\
2.9 \times 10^{10} \mathrm{~A} / \mathrm{m} / \mathrm{sec}(\text { commercial aluminum) }
\end{gathered}
$$

at time

$$
t \approx \begin{gathered}
0.6 \mu \mathrm{sec} \text { (stainless steel) } \\
10.8 \mu \mathrm{sec} \text { (commercial aluminum) }
\end{gathered}
$$

The voltage induced in an interior loop of area $A$ is approximately given by

$$
V \approx A \mu_{0} \frac{\partial}{\partial t} H_{i n}^{s t e p}(t)
$$

If we assume that the largest interior loop area is the interior diameter times the height $A \approx 2 h b \approx$ $1.1 \times 10^{-4} \mathrm{~m}^{2}$, we obtain the induced voltage

$$
V \approx \begin{gathered}
72 \text { volts (stainless steel) } \\
4 \text { volts (commercial aluminum) }
\end{gathered}
$$

These voltages, if they are induced in the output circuit of the transformer, are well below the threshold. On the other hand, if they are induced in the input circuit of the transformer (assuming the area of the input loop is not minimized and $A$ is representative), then the question must be 
asked whether the $1 \%$ worst case of 12 such pulses (see Figure 3 ), stepped up by the transformer, are capable of charging the capacitor to the threshold value. (Normal operation typically requires several thousand pulses.) Direct coupling to the transformer windings will be considered later in the report.

\section{CORNER EFFECT ENHANCEMENT OF PENETRANT MAGNETIC FIELD}

Barriers with corners, which are many skin depths thick, have been shown [8] to give larger field penetrations near the corners than would be anticipated from smooth shield walls. Since the pill box shield discussed here has corners, this effect must be addressed. (The transformer edges are in the proximity of the corners which is addressed more quantitatively by the two dimensional finite element models discussed later in the report.) Figure 5, from [8], shows the relative increase in interior field strength (as well as field direction) as a corner is approached. The field in this figure is time harmonic and the shield wall is several skin depths thick. The ordinate is the ratio of the interior field as a corner is approached to the approximately constant interior field removed from the corners. The abscissa is the ratio of the distance from the interior corner vertex to the interior dimension half width. The importance of this effect in the present problem, is that near the corner, the high frequency components of the penetrant magnetic field are enhanced, and hence the rise rate of the penetrant magnetic field is enhanced. Although approximations were made in Figure 5 (which break down as the abscissa becomes comparable to unity), we see that the interior corner field enhancement (and hence transient rise rate enhancement) only occurs in the immediate vicinity of the corner. Thus trace loops on the circuit board (using $x_{0}=2 \Delta \approx 2 \mathrm{~mm}$ in our case) should see little if any enhancement. The edge of the flyback transformer could see some field enhancement at its outer upper and lower edges. To obtain a feel for this enhancement we first note that the rise time of $\frac{\partial}{\partial t} H$ for the inverse exponential waveform is approximately $2 / \beta \approx 0.25 \mu \mathrm{sec}$ and the diffusion rise time of $\frac{\partial}{\partial t} H_{i n}^{s t e p}$ (for a step excitation) is [6] $\tau_{d} / 4 \approx 0.45 \mu \mathrm{sec}$ (for stainless steel) and $8.2 \mu \mathrm{sec}$ (for commercial aluminum) (note that the corner enhancement of the high frequencies will actually result in somewhat reduced diffusion rise times). Thus the diffusion time gives the order of the largest frequency of interest $\omega_{d} \approx 4 / \tau_{d}$ which gives a skin $\operatorname{depth} \delta=\sqrt{2 /\left(\omega_{d} \mu \sigma\right)} \approx \Delta / \sqrt{2}$. The enhancement shown in Figure 5 breaks down at distances within a skin depth of the corner (at which point we expect the value of the enhancement to saturate). Thus the abscissa value in Figure 5 is of order $\delta / x_{0} \approx 1 /(2 \sqrt{2}) \approx 0.35$ and the resulting enhancement factor in $\frac{\partial}{\partial t} H_{i n}^{\text {step }}$ is expected to be less than a factor of 2 (extending the curve to the left as a saturation value). Because this enhancement is less than a factor of two, and only exists near the upper and lower outer edges of the transformer, we expect this to have little or no effect on the transformer flux which links the windings.

\section{TRANSFORMER LOADING EFFECT ON SHIELD PENETRATION}

The effect of a high permeability transformer interior to the pill box barrier on the penetration of the shield needs to be assessed because it changes the interior load on the shield (it is a lower reluctance path provided there are not significant air gaps surrounding the transformer). To obtain an estimate for this effect we consider the case of a cylindrical shield of inner radius $b$, excited by a uniform magnetic field oriented perpendicular to the cylinder axis, containing limiting cases of $\mu_{0}$ material (no transformer or fully saturated transformer) and very high permeability material $\mu_{i}>>\mu_{0}$. The permeability will be allowed to become so large that we can approximate the boundary condition at the interior surface of the shield wall by that of a perfect magnetic conductor (PMC). A uniform time varying magnetic field perpendicular to the cylinder axis drives the problem. The frequency domain transfer function $T_{p}(p)$ [8], [6] is defined as the ratio of interior uniform magnetic field to 

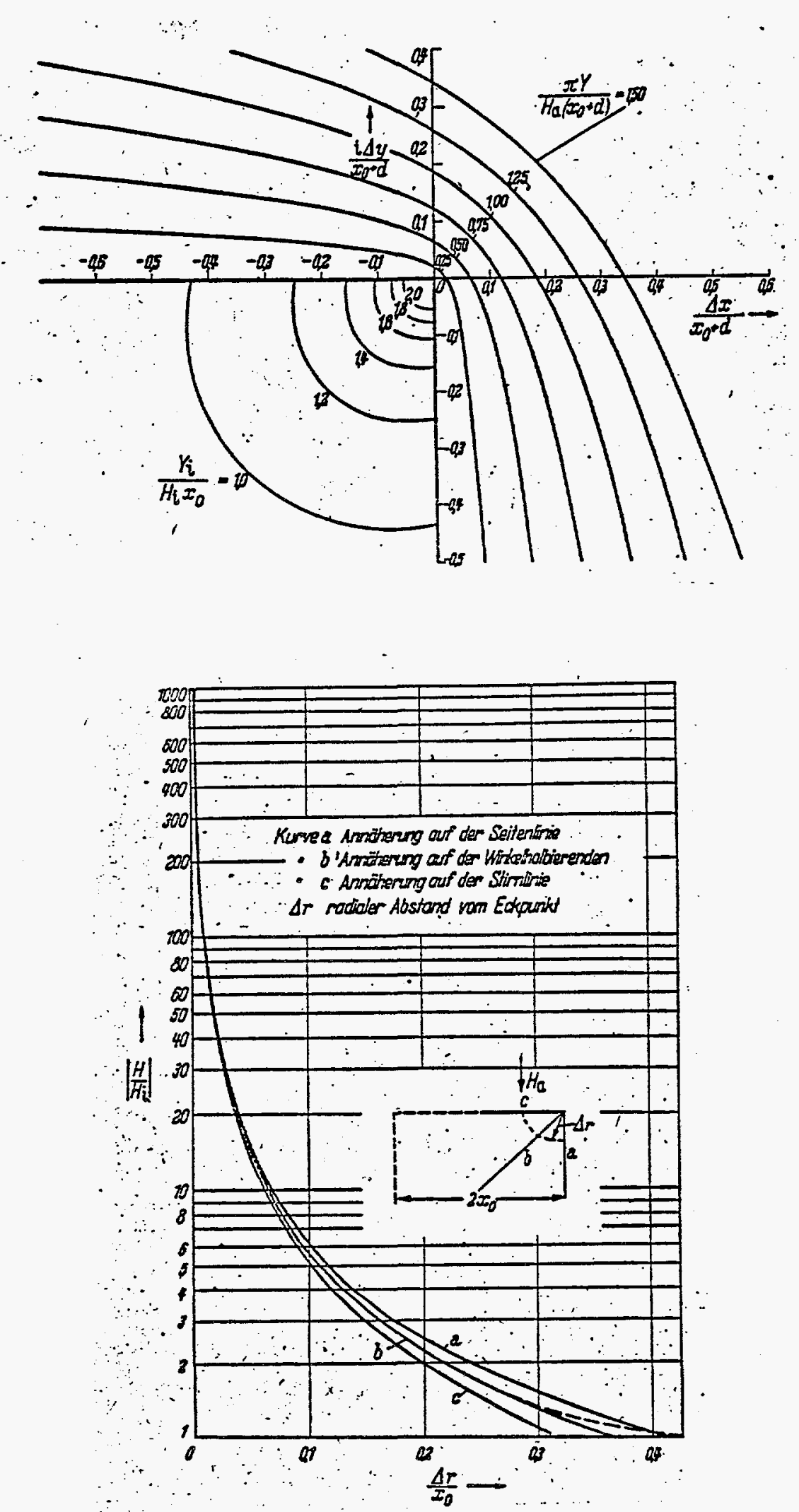

Figure 5. Enhancement of time harmonic (shield is several skin depths thick) magnetic field penetration at a right angle shield corner (from [8]). a) direction of exterior and interior magnetic fields, $b$ ) enhancement of penetrant magnetic field at corner divided by nominal interior magnetic field as a function of corner distance divided by interior half dimension. 
the exterior uniform (when the cylinder is not present) magnetic field. The case where material $\mu_{i}$ is present inside the cylinder is given by

$$
T_{p}(p)=\frac{1}{\left(\frac{K+K_{i}}{2 K}\right) \cosh (p)+\left(\frac{1+p^{2} K K_{i}}{2 K p}\right) \sinh (p)}
$$

where $p=\sqrt{s \tau_{d}}, s=-i \omega, K=\frac{\mu_{0} b}{\mu \Delta}, K_{i}=\frac{\mu_{i} b}{\mu \Delta}$, where $\mu$ is the shield wall magnetic permeability which we take to be $\mu_{0}$. The result for $\mu_{i}=\mu_{0}$ is [6]

$$
T_{p}(p)=\frac{1}{\cosh (p)+\frac{1}{2}\left(K p+\frac{1}{K p}\right) \sinh (p)}
$$

The corresponding result for the interior PMC (or high permeability material $\mu_{i} / \mu_{0} \rightarrow \infty$ ) is

$$
T_{p}(p)=\frac{\mu_{0} / \mu_{i}}{\frac{1}{2} \cosh (p)+\frac{1}{2} K p \sinh (p)}
$$

The presence of the large factor $K$ (for many shields this is quite large but here it is order four) slows the step excitation rise rate of the magnetic field in the cases of the $\mu_{0}$ interior and the PMC interior. The derivative of the magnetic field still reaches a peak in $\approx \tau_{d} / 4$ to $\tau_{d} / 3$. There appears to be little enhancement in interior magnetic flux density $B$ gained by the PMC case, over that of the $\mu_{0}$ case, at early time.

\section{CENTRAL FOIL PENETRATION}

The central $2 b_{f}=7 \mathrm{~mm}$ diameter of the bottom plate has a thickness of $\Delta_{f}=0.1 \mathrm{~mm}$. This thin region thus allows much earlier penetration of the magnetic field. The diffusion time associated with this thickness is $\tau_{d} \approx 18 \mathrm{nsec}$ for stainless steel to $\tau_{d} \approx 0.33 \mu \mathrm{sec}$ for commercial aluminum. The skin depth $\delta=\sqrt{2 /(\omega \mu \sigma)}$ is larger than the thickness $\Delta_{f}$ for all significant lightning frequencies. Thus a thin sheet impedance description of this region is appropriate

$$
Z_{s} \approx \frac{1}{\sigma \Delta_{f}}
$$

The interior magnetic field in the pill box (away from the central foil region) can be assessed by use of the low frequency magnetic dipole moments of an impedance loaded circular aperture of radius $b_{f}=3.5 \mathrm{~mm}$. The fact that the height $h \approx 4 \mathrm{~mm}$ is larger than the aperture radius, allows us to ignore the loading effect of the backup ground plane as far as determining the aperture dipole moments or polarizabilities [6]. The unloaded circular aperture has magnetic polarizability [6]

$$
\alpha_{m 0}=\frac{4}{3} b_{f}^{3}
$$

The impedance loaded aperture has polarizability [6]

$$
\alpha_{m} \approx \alpha_{m 0} /\left(1-i \omega \tau_{f}\right)
$$

The time constant $\tau_{f}$ is $[6]$

$$
\tau_{f}=\frac{2}{3 \pi} L_{f} / Z_{s}
$$

where the equivalent inductance $L_{f}$ can be approximated by [6] 


$$
L_{f} \approx \mu_{0} A / P=\mu_{0} b_{f} / 2
$$

where here $A$ and $P$ are the area and perimeter of the circular aperture. The aperture time constant is $\tau_{f} \approx \mu_{0} \sigma b_{f} \Delta_{f} /(3 \pi) \approx 1.2 \mu \mathrm{sec}$ for commercial aluminum and $\tau_{f} \approx 65$ nsec for stainless steel. Thus, for stainless steel the time constant of the foil is much less than the rise time of the lightning field, and the hole may be treated without the loading using $\alpha_{m 0}$. The aluminum foil may be treated using $\alpha_{m}$ but step function excitation (or decaying exponential) may be used since the foil time constant is several times larger than the lightning field rise time.

The (imaged) magnetic dipole moment is given by

$$
\underline{m}=-2 \alpha_{m} \underline{H}_{s c}
$$

where $\underline{H}_{s c}$ is the exterior short circuit magnetic field at the center of the hole. We can take $\underline{H}_{s c}=H_{s c} \underline{e}_{x}$ without loss of generality and thus $m_{x}=-2 \alpha_{m} H_{s c}$. Let the magnetic field be determined by means of a scalar potential

$$
\underline{H}=-\nabla \phi_{m}
$$

The imaged magnetic dipole moment (27) resides at $z=0$ (where $z$ is the axis of the cylindrical pill box) and can be imaged in the flat walls to form an infinite array at locations $z=2 n h$, $(n=0, \pm 1, \pm 2, \ldots)$. To determine the dominant $x-y$ components of the field in the pill box (away from the hole and not too near the outer wall) we can approximate the interior excitation as a magnetic line dipole moment

$$
\underline{m}_{2}=\frac{1}{2 h} \underline{m}
$$

The potential of this line dipole is

$$
\phi_{m}=\frac{\underline{m}_{2} \cdot \underline{\rho}}{2 \pi \rho^{2}}=\frac{m_{2 x} \cos \varphi}{2 \pi \rho}
$$

The components of the magnetic field are thus

$$
\begin{aligned}
& H_{\rho}=\frac{m_{2 x} \cos \varphi}{2 \pi \rho^{2}} \\
& H_{\varphi}=\frac{m_{2 x} \sin \varphi}{2 \pi \rho^{2}}
\end{aligned}
$$

Using $\alpha_{m 0}$ for stainless steel we find

$$
H_{i n}=\frac{2 b_{f}^{3}}{3 \pi h \rho^{2}} H_{s c} \text { (stainless steel) }
$$

Evaluating the time derivative of $(32)$, using $(\beta / 4) H_{0}$ for the peak of the inverse exponential waveform, gives

$$
\frac{\partial}{\partial t} H_{\text {in }} \approx 2 \times 10^{11} \mathrm{~A} / \mathrm{m} / \mathrm{sec} \text { (stainless steel) }
$$

at a distance $\rho=7 \mathrm{~mm}$ (which is a reasonable distance at which to evaluate the foil contribution, note $b_{f}=3.5 \mathrm{~mm}<\rho<b=13.5 \mathrm{~mm}$, since it is on the middle region of the circuit board and at the inner edge of the transformer). Comparing (33) with the result (15) shows that this contribution is 
generally smaller. Furthermore, the transverse direction of this field (in the absence of perturbing objects) will not couple well to traces in a constant $z$ plane, nor to the transformer. In addition the exciting field amplitude (7) is an upper bound, since the lighting current line source is constrained physically to the top side of the pill box (the side opposite the foil). Thus for stainless steel the foil hole contribution appears to be smaller than the direct diffusive contribution.

The aluminum foil contribution can be determined by noting the inverse transform

$$
F(t)=\frac{1 / \tau_{f}}{2 \pi} \int_{-\infty}^{\infty} e^{-i \omega t} \frac{d \omega}{(\alpha-i \omega)\left(1 / \tau_{f}-i \omega\right)}=\frac{1}{\left(1-\alpha \tau_{f}\right)}\left(e^{-\alpha t}-e^{-t / \tau_{f}}\right) u(t) \approx\left(e^{-\alpha t}-e^{-t / \tau_{f}}\right) u(t)
$$

The magnetic field is thus

$$
H_{i n}=\frac{2 b_{f}^{3}}{3 \pi h \rho^{2}} H_{0} F(t) \text { (commercial aluminum) }
$$

Evaluating the peak time derivative as $H_{0} / \tau_{f}$ gives

$$
\frac{\partial}{\partial t} H_{\text {in }} \approx 8.5 \times 10^{10} \mathrm{~A} / \mathrm{m} / \mathrm{sec} \text { (commercial aluminum) }
$$

The result (36) should be compared to the result (15) for aluminum. Thus the foil penetration is nearly three times larger than the direct diffusive penetration. The same comments as for the stainless steel case apply, however. Therefore, although the foil penetration for aluminum is not negligible, it appears to be comparable to the direct diffusive penetration (which is small compared to the stainless steel results).

It is instructive to consider the $H_{z}$ component of the magnetic field in the absence of perturbing obstacles. we note that the potential resulting from the magnetic dipole is

$$
\phi_{m}=\frac{\underline{m} \cdot \underline{r}}{4 \pi r^{3}}=\frac{m_{x} x}{4 \pi r^{3}}
$$

The Green's function problem $\nabla^{2} G=-\delta\left(\underline{r}-\underline{r}^{\prime}\right)$ has solution $G=1 /\left(4 \pi\left|\underline{r}-\underline{r}^{\prime}\right|\right)$. Thus the dipole problem can be written as

$$
\nabla^{2} \phi_{m}=-m_{x} \frac{\partial}{\partial x^{\prime}} \delta\left(\underline{r}-\underline{r}^{\prime}\right)=m_{x} \frac{\partial}{\partial x} \delta\left(\underline{r}-\underline{r}^{\prime}\right)=m_{x} \frac{\partial}{\partial x} \delta(\underline{r})
$$

Consider only the top and bottom surfaces $z= \pm h$ for which the boundary conditions are

$$
\frac{\partial}{\partial z} \phi_{m}=0
$$

The Green's function for this problem can be written as

$$
G=-\frac{1}{4 \pi h} \ln (\rho)+\frac{1}{2 \pi h} \sum_{n=1}^{\infty} \cos (n \pi z / h) K_{0}(n \pi \rho / h)
$$

Thus we obtain

$$
\phi_{m}=-m_{x} \frac{\partial}{\partial x} G=\frac{m_{x} \cos \varphi}{4 \pi h \rho}+\frac{m_{x}}{2 h^{2}} \cos (\varphi) \sum_{n=1}^{\infty} n \cos (n \pi z / h) K_{1}(n \pi \rho / h)
$$

The magnetic field $H_{z}$ is thus 


$$
H_{z}=\frac{\pi m_{x}}{2 h^{3}} \cos (\varphi) \sum_{n=1}^{\infty} n^{2} \sin (n \pi z / h) K_{1}(n \pi \rho / h) \sim \frac{\pi m_{x}}{2 h^{3}} \sqrt{\frac{h}{2 \rho}} \cos (\varphi) \sin (\pi z / h) e^{-\pi \rho / h}, \pi \rho>>h
$$

which makes clear the exponential decay in this component if perturbing obstacles are not present.

\section{WORST CASE NEARBY WIRE}

Now consider the worst case of a wire carrying the lightning current immediately adjacent to the outside surface of the top plate of the pill box and a loop immediately adjacent to the inside surface at the same point as shown in Figure 2. (Direct attachment with normal orientation of the lightning current, with respect to the shield wall, results in smaller voltages [9], [10].) The loop is assumed to be large enough (the return leg is theoretically at infinite distance from the wall, but the distance $h+\Delta=5 \Delta$ is far enough to approximately achieve this result) to capture the majority of penetrant magnetic flux along its length $\ell$, which here we take to be the diameter $\ell \approx 2 b$. The voltage induced in the loop by a step function current is approximately [11]

$$
V \approx \frac{4 \ell I_{0}}{\pi \sigma \Delta^{2}}\left(\frac{\tau_{d}}{4 t}\right) e^{-\tau_{d} /(4 t)} \frac{1}{1+\left(\mu / \mu_{0}\right) \sqrt{\pi t / \tau_{d}}}
$$

The peak value for $\mu=\mu_{0}$ is approximately

$$
V \approx \frac{4 \ell I_{0}}{\pi e \sigma \Delta^{2}}(0.54)
$$

and occurs at time

$$
\tau_{d} /(4 t) \approx 1.2
$$

Figure 6 , from [11], shows a comparison of the exact solution with the approximate results (43), (44), (45). The worst case induced voltage is

$$
V \approx \begin{gathered}
976 \text { volts (stainless steel) } \\
52.5 \text { volts (commercial aluminum) }
\end{gathered}
$$

The stainless steel result, if induced in the output circuit, is above the threshold voltage. However it should be noted that an insulated wire, carrying maximum lightning current, could not have zero radius; a radius of, say, $1 \mathrm{~mm}$ is probably a more appropriate number. Furthermore, the interior traces are not on the wall; a spacing again of $1 \mathrm{~mm}$ is probably more appropriate for the trace clearance. A resultant spacing of $\rho_{0} \approx 3 \mathrm{~mm}$ between the wire current centroid and the interior loop is thus more reasonable. Figure 7 shows a comparison of the exact interior magnetic field in the geometry of Figure 2 (but with a distance $\rho_{0}$ between the source filament and the observation point) and the magnetic field resulting from a simple thin sheet impedance description. $Z_{s}=1 /(\sigma \Delta)$, where $E_{z}=Z_{s}\left(H_{x}^{-}-H_{x}^{+}\right)$, of the conducting layer (step function current excitation is used in both solutions). The thin sheet impedance approximation improves in accuracy as $\rho_{0} / \Delta$ increases from unity. The time derivative of the thin sheet impedance magnetic field is

$$
\frac{\partial}{\partial t} H_{i n}^{\text {step }}(t) \approx \frac{I_{0}}{2 \pi \rho_{0}} \frac{\rho_{0} \mu_{0} \sigma \Delta /\left(2 t^{2}\right)}{\left[1+\rho_{0} \mu_{0} \sigma \Delta /(2 t)\right]^{2}} \leq \frac{I_{0}}{\pi \rho_{0}^{2} \mu_{0} \sigma \Delta}
$$

If we multiply $\mu_{0}$ and by the interior wire loop length taken to be the interior pill box diameter $\ell=2 b$, and integrate in $\rho_{0}$ from the close leg of the interior wire loop $\rho_{0} \approx 3 \mathrm{~mm}$ to infinity we obtain 

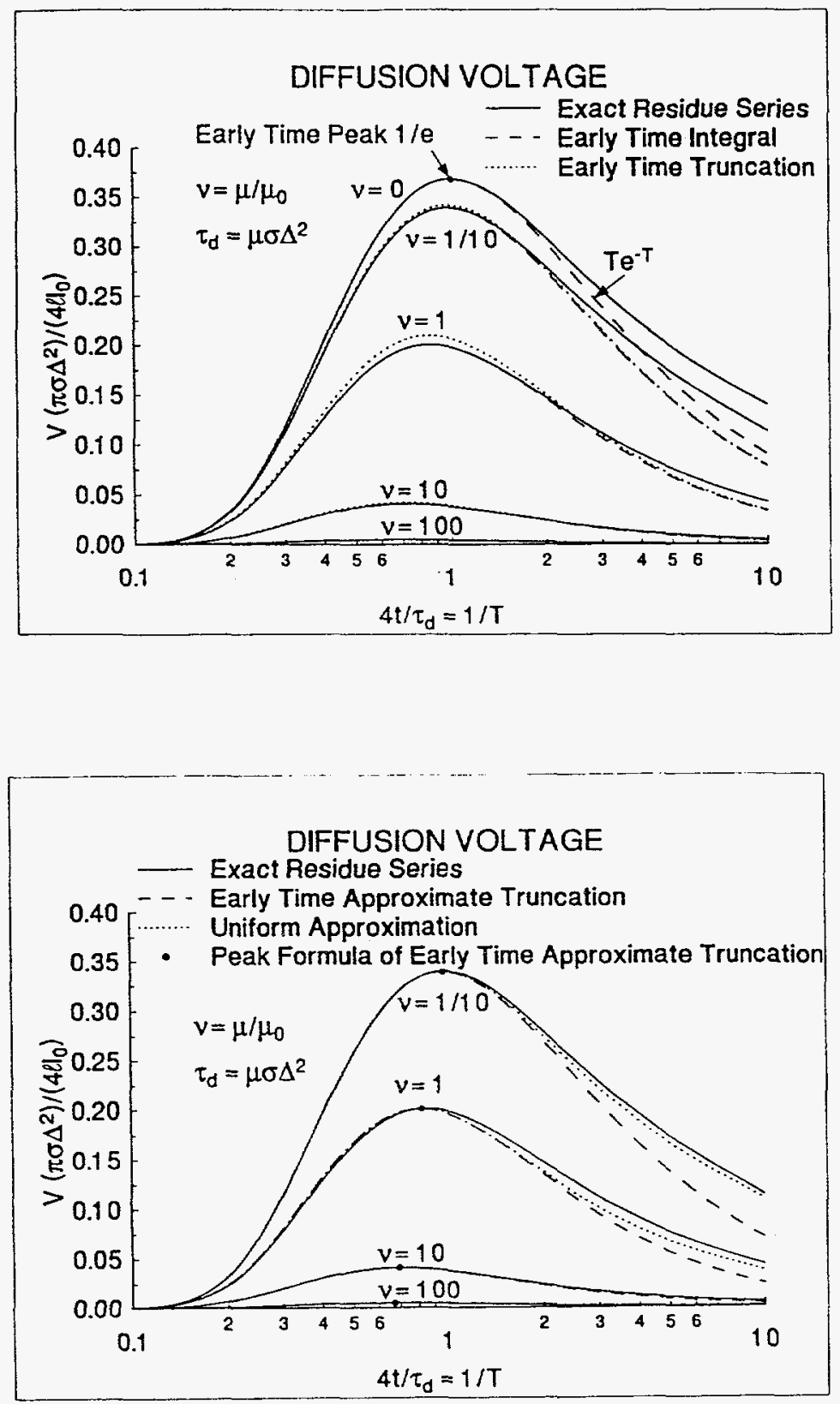

Figure 6. Comparison of exact and approximate (based on an averaging approximation to an integral) representations of the induced voltage for the worst case geometry of Figure 2. 


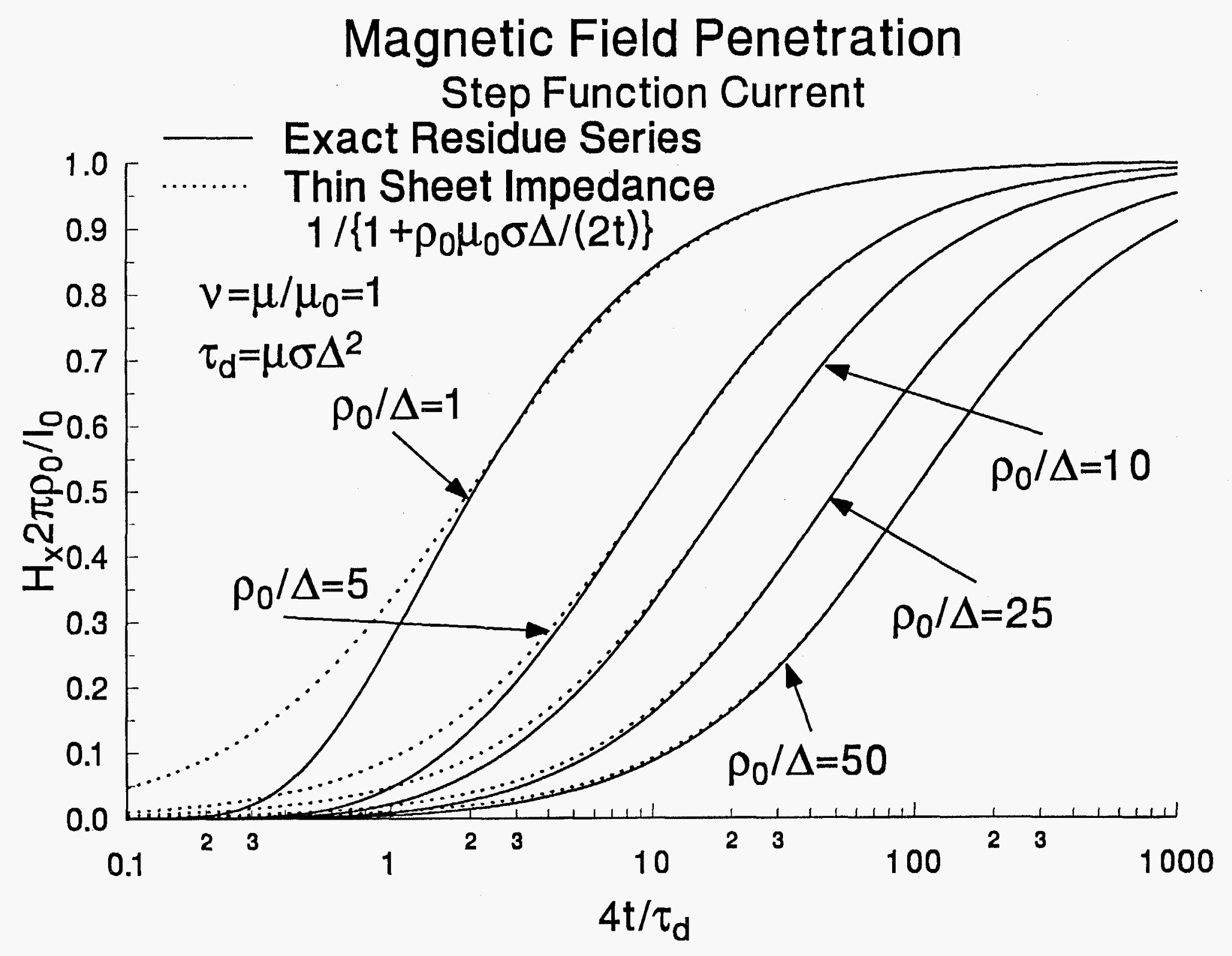
for the interior magnetic field for the geometry of Figure 2 (except $\rho_{0} \geq \Delta$ is the distance from the source filament to the observation point). 


$$
V \approx \frac{I_{0} \ell}{\pi \sigma \Delta \rho_{0}} \approx \begin{gathered}
409 \text { volts (stainless steel) } \\
22 \text { volts (aluminum) }
\end{gathered}
$$

which are below the threshold. (Again, the question needs to be answered if twelve pulses of this magnitude on the input circuit, could result in voltages of concern in the output circuit of the transformer.) Note that if we take $\rho_{0}=\Delta,(48)$ gives $V \approx 1228$ volts (stainless steel) and 66 volts (commercial aluminum); thus even for the closest spacing between source filament and loop, the impedance solution is a reasonable estimate (and is slightly greater than the value) of the accurate solution (46).

Because there is another conducting wall of the pill box at a distance $\rho_{0}^{(f a r)}=6 \mathrm{~mm}$ from the source one might be concerned that the penetrant magnetic field is underestimated by the single infinite plane problem (for example if we take the bottom wall of the pill box to be perfectly conducting and treat it as a second conducting plane, we can image the problem about the surface at $\rho_{0}^{(f a r)}$. We expect roughly a doubling of the field at the surface $\rho_{0}^{(f a r)}$. Therefore, taking twice the field (47), and integrating from the close $\operatorname{leg} \rho_{0}^{(\text {close })} \approx 3 \mathrm{~mm}$ to the far leg of the interior wire loop $\rho_{0}^{(f a r)} \approx 6 \mathrm{~mm}$, we obtain the voltage

$$
V \approx \frac{2 I_{0} \ell}{\pi \sigma \Delta}\left(\frac{1}{\rho_{0}^{(\text {close })}}-\frac{1}{\rho_{0}^{(f a r)}}\right) \approx \begin{gathered}
409 \text { volts }(\text { stainless steel }) \\
22 \text { volts }(\text { commercial aluminum })
\end{gathered}
$$

the same result.

\section{TRANSFORMER COUPLING}

Next we consider direct magnetic field coupling to the transformer shown in Figure 8 . The shape is cylindrical with height $h_{t} \approx 4 \mathrm{~mm}$ and radius $b_{t} \approx 3.5 \mathrm{~mm}$. As an approximation we consider immersing the transformer in a plane field $H_{\text {in }}$. Let us assume first that the field is below the saturation field of the ferrite material. Assuming that the permeability of the ferrite material is large we can use as an approximation the external perfect magnetic conductor boundary condition

$$
\underline{n} \times \underline{H}=0
$$

where $\underline{n}$ is the outward unit normal to the surface of the transformer. The question then is: how much magnetic flux is captured by the highly permeable transformer.

Let us take the uniform magnetic field $\underline{H}_{i n}$ to be oriented along the axis of the transformer. A canonical shape which admits a closed form solution is that of the oblate spheroid. The relation between cylindrical and spheroidal coordinates (in an axisymmetric problem) is

$$
\begin{gathered}
\rho=c_{1} \sqrt{\left(1+\zeta^{2}\right)\left(1-\xi^{2}\right)} \\
z=c_{1} \zeta \xi
\end{gathered}
$$

Thus we obtain $b_{t}=c_{1} \sqrt{1+\zeta_{0}^{2}}$ and $h_{t} / 2=c_{1} \zeta_{0}$. Solving for $\zeta_{0}$ gives

$$
\zeta_{0}=1 / \sqrt{\left(2 b_{t} / h_{t}\right)^{2}-1} \approx 0.6963
$$

Using duality (to convert from an electric field problem to a magnetic field problem) from Smythe [12] or Weber [13] a spheroid with magnetic permeability $\mu$ at coordinate location $\zeta=\zeta_{0}$ has uniform internal field 

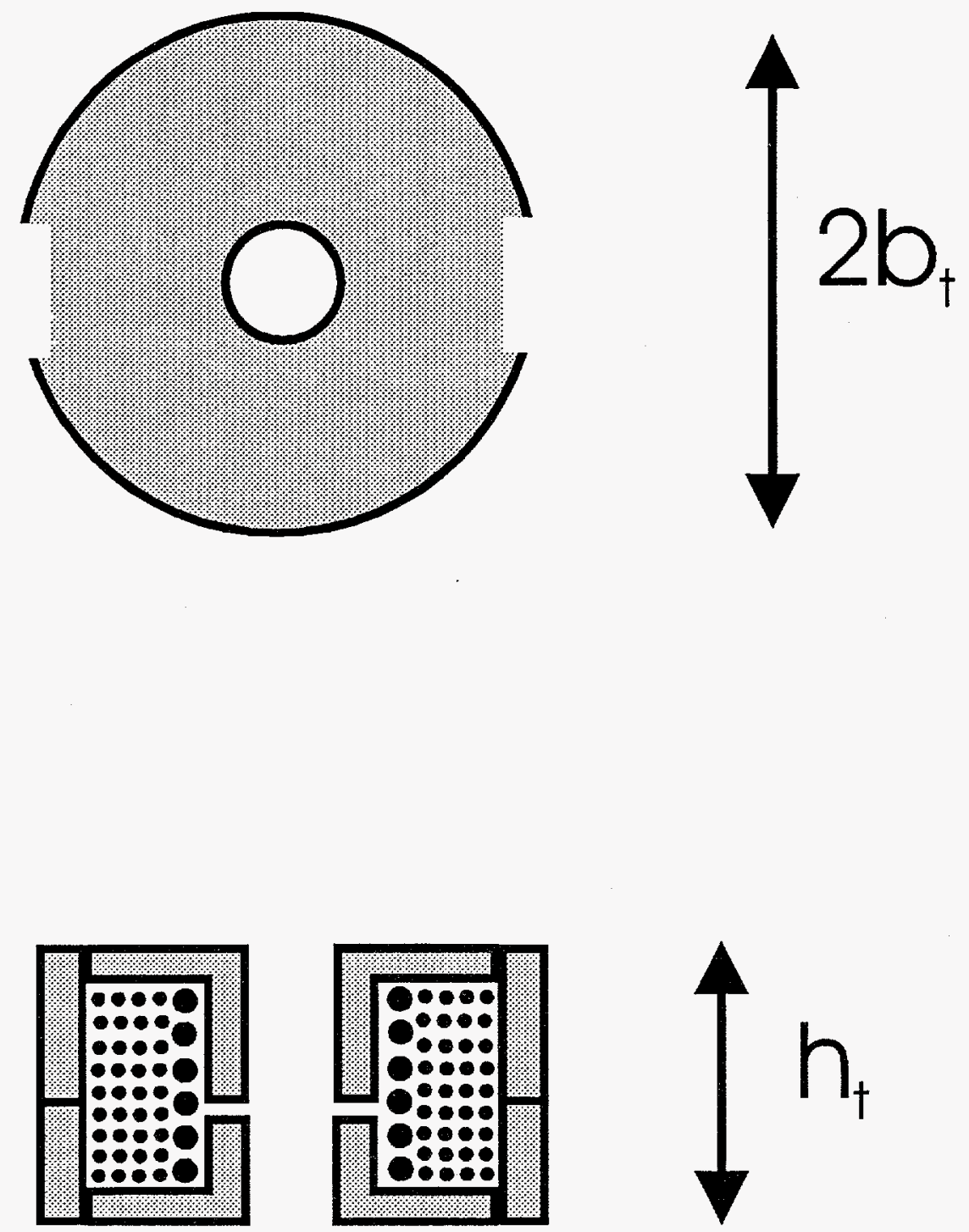

Figure 8. Geometry of transformer. 


$$
H=H_{i n} /\left[\nu-(\nu-1) \zeta_{0}\left\{\left(1+\zeta_{0}^{2}\right) \operatorname{Arctan}\left(\zeta_{0}\right)-\zeta_{0}\right\}\right]
$$

where

$$
\nu=\mu / \mu_{0}
$$

The cross sectional area of the spheroid is

$$
\pi b_{t}=\pi c_{1}^{2}\left(1+\zeta_{0}^{2}\right)
$$

Because the internal field is uniform, the magnetic flux $\Phi$, intercepted by the spheroid, can be found as

$$
\Phi /\left(\pi b_{t}^{2} \mu_{0} H_{i n}\right)=1 /\left[1-(1-1 / \nu) \zeta_{0}\left\{\left(1+\zeta_{0}^{2}\right) \operatorname{Arccot}\left(\zeta_{0}\right)-\zeta_{0}\right\}\right]
$$

It is interesting to note that the spherical limit is

$$
\Phi /\left\{\pi c_{1}^{2}\left(1+\zeta_{0}^{2}\right) \mu_{0} H_{i n}\right\} \sim 3 /(1+2 / \nu), h_{t} \rightarrow 2 b_{t} \text { (sphere) }
$$

Applying duality to the formula in Stratton [14] for a sphere of radius $b_{t}$ we directly obtain $\Phi /\left(\pi b_{t}^{2} \mu_{0} H_{i n}\right)=3 /(1+2 / \nu)$, the same result.

For large internal magnetic permeability $\nu \rightarrow \infty$ (the boundary condition (50) is satisfied) and (53) and (56) become

$$
\begin{gathered}
H \sim \frac{1}{\nu} H_{i n} /\left[1-\zeta_{0}\left\{\left(1+\zeta_{0}^{2}\right) \operatorname{Arccot}\left(\zeta_{0}\right)-\zeta_{0}\right\}\right] \\
\Phi /\left(\pi b_{t}^{2} \mu_{0} H_{i n}\right) \sim 1 /\left[1-\zeta_{0}\left\{\left(1+\zeta_{0}^{2}\right) \operatorname{Arccot}\left(\zeta_{0}\right)-\zeta_{0}\right\}\right] \approx 2.0423
\end{gathered}
$$

Thus the magnetic flux captured is about twice the cross sectional area times the ambient magnetic flux density $\Phi \approx 2 \pi b_{t}^{2} \mu_{0} H_{i n}$.

We now consider the interior of the transformer as a magnetic circuit. Figure 8 shows the transformer geometry. Figure 9 [15] gives data sheets on a typical pot core and the properties of the ferrite material used. We take the magnetic flux (59) to couple into the transformer from top to bottom. The flux splits between the inner magnetic path linking the transformer windings and the outer magnetic path. The air gap of the inner path leads to a higher reluctance (when the material is unsaturated). The reluctance is defined by [16]

$$
m m f=R \Phi
$$

where the magnetomotive force is the line integral of the magnetic field

$$
m m f=\int_{C} \underline{H} \cdot \underline{d \ell}
$$

Assuming that each leg of the transformer has the flux confined and approximately uniform in the corresponding cross section $S$ we find for the approximate reluctance

$$
R=\frac{\ell}{\mu S}
$$

The $m m f$ over the two parallel paths must be equal. Thus we can find the portion of the flux that links the windings. Let $\Phi_{w}$ be the magnetic flux linking the windings and $\Phi_{b}$ be the flux in the 


\section{SERIES 704}

Mechanical Data

Effective Core Set Parameters

\begin{tabular}{|l|c|l|}
\hline MAGNETIC PATH LENGTH & $\ell_{e}$ & $\begin{array}{l}.39 \mathrm{in} . \\
1.0 \mathrm{~cm}\end{array}$ \\
\hline CORE CONSTANT & $\sum \frac{\ell_{e}}{A_{e}}$ & $\begin{array}{l}36.3 \mathrm{in} \cdot{ }^{1} \\
14.3 \mathrm{~cm}^{-1}\end{array}$ \\
\hline EFFECTIVE CORE AREA & $A_{e}$ & $\begin{array}{l}.011 \mathrm{in}^{2} \\
.07 \mathrm{~cm}^{2}\end{array}$ \\
\hline $\begin{array}{l}\text { EFFECTIVE CORE } \\
\text { VOLUME }\end{array}$ & $v_{e}$ & $\begin{array}{l}.0043 \mathrm{in}^{3} \\
.07 \mathrm{~cm}^{3}\end{array}$ \\
\hline WEIGHT & & $\begin{array}{l}.0018 \mathrm{oz} . \\
.5 \mathrm{Grams}\end{array}$ \\
\hline
\end{tabular}

NOTE: MINIMUM CORE AREA $.050 \mathrm{~cm}^{2}$

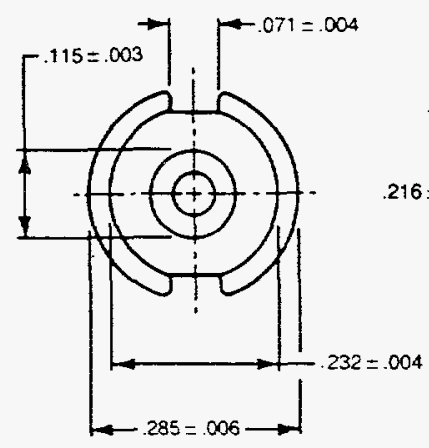

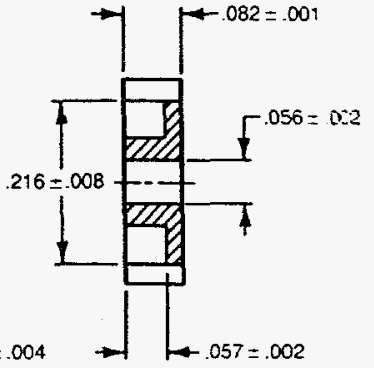

704 PLDO

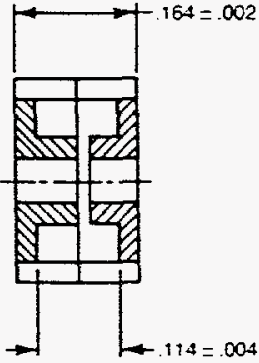

704PA

\section{Electrical Data}

Ungapped Pot Cores

\begin{tabular}{|c|c|c|c|}
\hline $\begin{array}{c}\text { CORE } \\
\text { PART NO. }\end{array}$ & $\begin{array}{c}\text { CORE } \\
\text { MATERIAL }\end{array}$ & $\begin{array}{c}A_{L} \dagger \\
\pm 25 \%\end{array}$ & $\begin{array}{c}\mu_{e} \dagger \\
\text { (REF.) }\end{array}$ \\
\hline 704 PLOO-3B7 & $3 B 7$ & 1150 & 1315 \\
\hline 704 PLO0-3B9 & $3 B 9$ & 1000 & 1140 \\
\hline
\end{tabular}

- Part number for a core half.

tPer pair of cores.

Figure 9. Data sheets on 704 pot core transformer (from [15]). a) Geometry and electrical data of ferrite core. Sheet 1 of 2. 
Gapped Pot Cores for Frequency Selective Applications

\begin{tabular}{|c|c|c|c|c|c|c|}
\hline \multirow{2}{*}{$\begin{array}{c}\text { NON- } \\
\text { ADJUSTABLE } \\
\text { GAPPED } \\
\text { POT CORE } \\
\text { PART NO. } \\
\end{array}$} & \multirow[t]{2}{*}{$\begin{array}{c}\text { CORE } \\
\text { MATERIAL }\end{array}$} & \multirow{2}{*}{$\begin{array}{l}\text { ALt } \\
\text { VALUE } \\
\pm 3 \%\end{array}$} & \multirow[t]{2}{*}{ (REF.) } & \multirow{2}{*}{$\begin{array}{l}\text { APPROX. } \\
\text { GAP } \\
\text { LENGTH } \\
\text { (in.) }\end{array}$} & \multicolumn{2}{|c|}{$\begin{array}{l}\text { TEMPERATURE } \\
\text { COEFFICIENT PPM/CC }\end{array}$} \\
\hline & & & & & MIN-MAX & TEMP. RANGE \\
\hline 704 PA $100-3 B 7$ & \multirow{2}{*}{$\begin{array}{c}387 \\
\text { (To } 300 \mathrm{kHz} \text { ) }\end{array}$} & 100 & 114 & .004 & $\begin{array}{l}-68 \text { to } \\
+68 \\
\end{array}$ & \multirow{2}{*}{$\begin{array}{l}+20^{\circ} \\
\text { to } \\
+70^{\circ} \mathrm{C}\end{array}$} \\
\hline 704 PA $160-3 B 7$ & & 160 & 183 & .002 & $\begin{array}{c}-109 \text { to } \\
+109\end{array}$ & \\
\hline 704PA100-3B9 & \multirow{2}{*}{$\begin{array}{c}3 \mathrm{~B} 9 \\
\text { (To } 300 \mathrm{kHz} \text { ) }\end{array}$} & 100 & 114 & .004 & $\begin{array}{c}+102 \text { to } \\
+217\end{array}$ & \multirow{2}{*}{$\begin{aligned} &-30^{\circ} \\
& \text { to } \\
&+ 70^{\circ} \mathrm{C}\end{aligned}$} \\
\hline 704PA $160-389$ & & 160 & 183 & .002 & $\begin{array}{l}+164 \text { to } \\
+348\end{array}$ & \\
\hline
\end{tabular}

- Part number is for a core set ( 2 cores).

tThe $A_{L}$ values are based on a fully wound bobbin.

\section{Standard Bobbin}
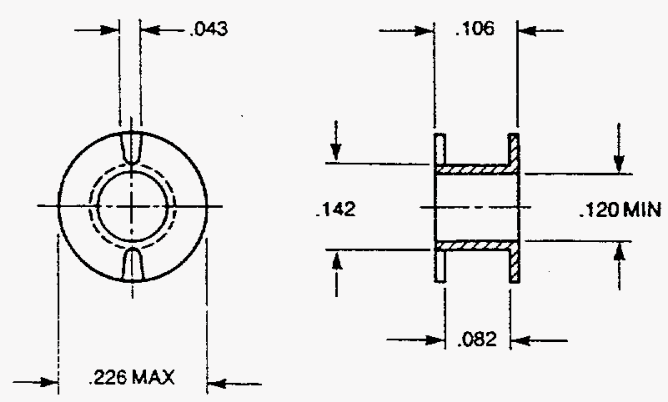

\section{PART NO. 704FIN}

Material: Glass filled Nylon

Max. Operating Temp: $120^{\circ} \mathrm{C}$

Winding Area .0034 in. ${ }^{2}$

Mean Length of Turn .57 in.

Flammability: UL 94-VO

$A_{L}$ vs. DC Bias Curves
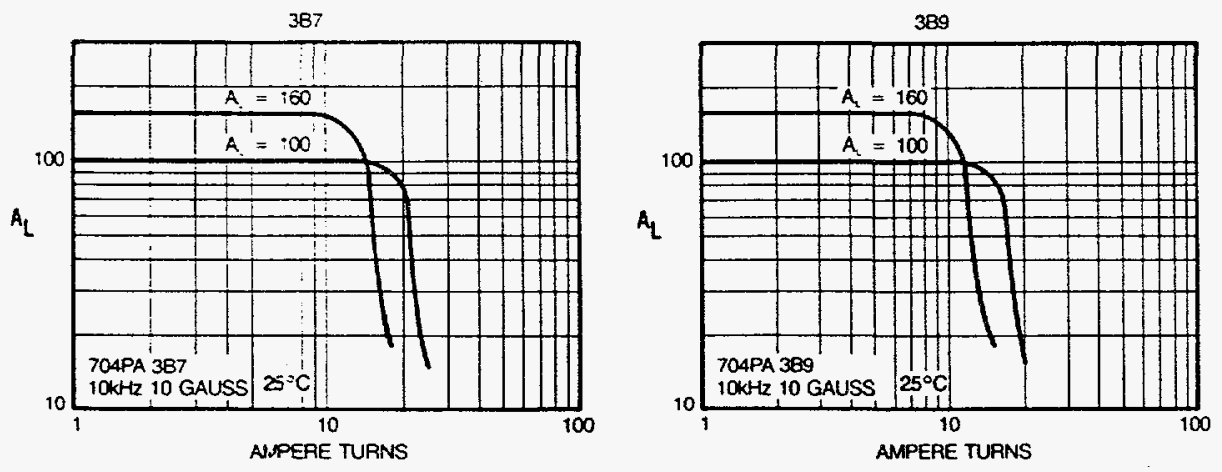

Figure 9. Data sheets on 704 pot core transformer (from [15]). a) Geometry and electrical data of ferrite core. Sheet 2 of 2 . 


\section{SERIES 3B7
FERRITE MATERIALS}

3B7 Material

Due to its low Temperature Factor (TF), our 3B7 Manganese Zinc ferrite is particularly useful in low temperature coefficient devices or for inductors to be used with low TC capacitors. Frequency range from audio to $300 \mathrm{kHz}$.

The low losses under high flux density condition along with the high B saturation make 3B7 material very attractive for pulse and power transformer applications.

Available in:

POT CORES

TOROIDS

\section{B7 Characteristics}

Parameters shown are based upon measurements of a medium toroidal core.

\section{Initial Permeability}

$$
\text { at } 25^{\circ} \mathrm{C}
$$

Saturation Flux Density at $25^{\circ} \mathrm{C}$.,

$H=2$ oersted

Coercive Force

Loss Factor at $B \leqslant 1$ gauss $100 \mathrm{kHz}$ $500 \mathrm{kHz}$

$1 \mathrm{MHz}$

Temperature Factor $\left(20^{\circ}-70^{\circ} \mathrm{C}\right)$

Disaccommodation Factor (10-100 minutes)

Curie Temperature

\begin{tabular}{|c|c|}
\hline$\mu_{1}$ & $2300( \pm 20 \%)$ \\
\hline$B_{s}$ & 3800 gauss* \\
\hline$H_{c}$ & 0.2 oersted \\
\hline$\mu_{i}$ & $\begin{array}{r}\leqslant 5 \times 10^{6} \\
25 \times 10^{6} \\
120 \times 10^{6}\end{array}$ \\
\hline TF & $\begin{array}{l}-0.6 \times 10^{-5} \mathrm{Min} . \\
+0.6 \times 10^{-6} \mathrm{Max}\end{array}$ \\
\hline DF & $<3.5 \times 10^{-6}$ \\
\hline$T_{c}$ & $\geqslant 170^{\circ} \mathrm{C}$ \\
\hline
\end{tabular}

"Typical values
Initial Permeability $\left(\mu_{i}\right)$ vs. Frequency

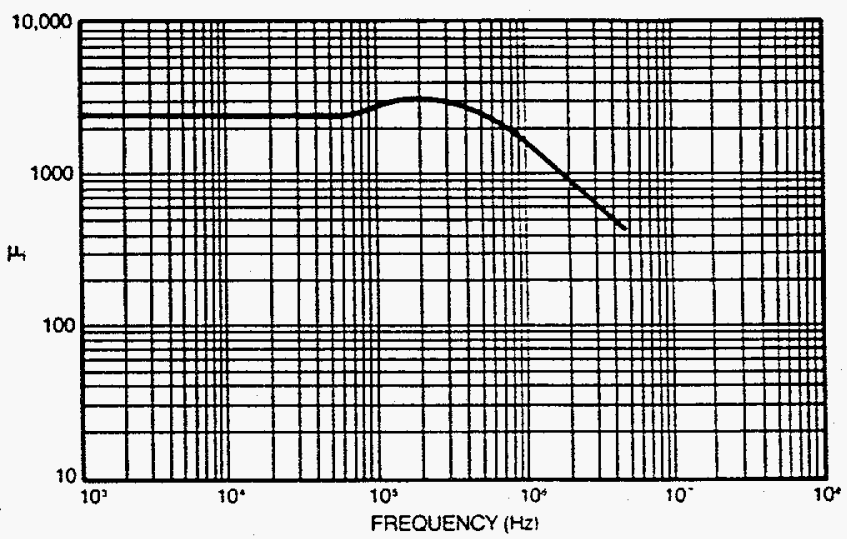

Permeability $\left(\mu_{2}\right)$ vs. Flux Density $(\hat{B})$

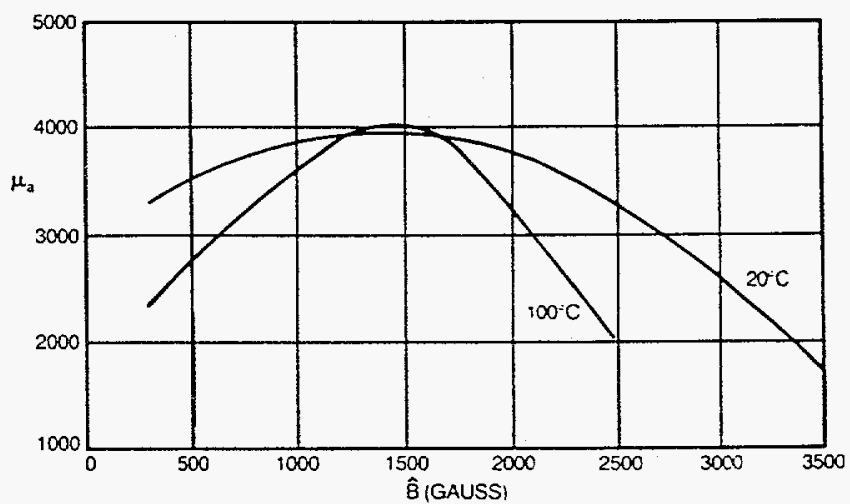

Initial Permeability $\left(\mu_{1}\right)$ vs. Temperature

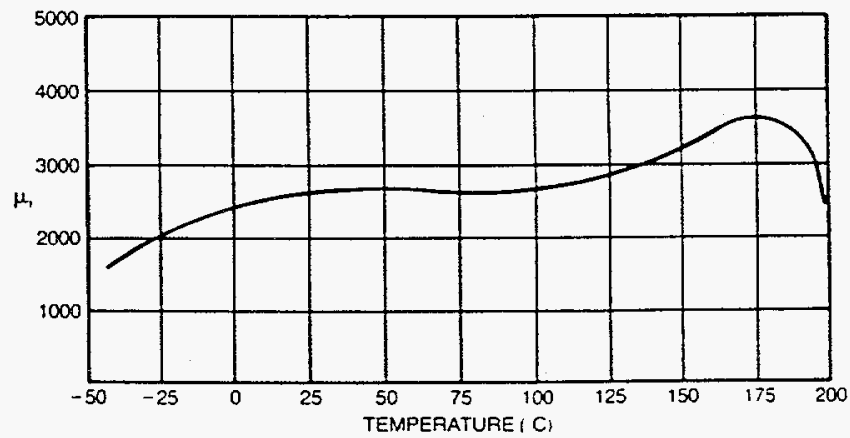

Figure 9. Data sheets on 704 pot core transformer (from [15]). b) Magnetic properties of ferrite materials. Sheet 1 of 2 . 


\section{SERIES 3B7 FERRITE MATERIALS}

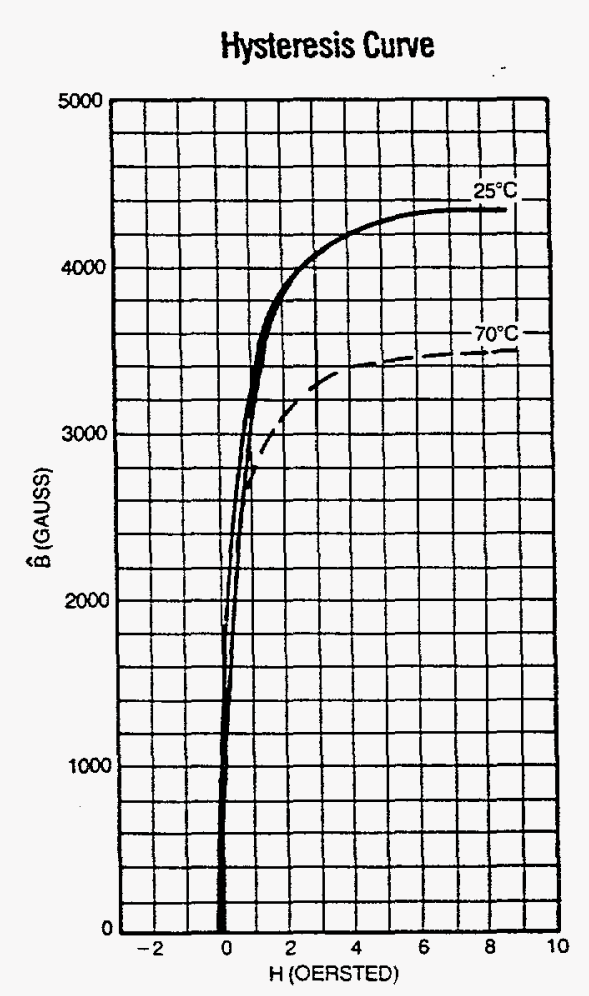

\section{Core Loss vs. Flux Density}

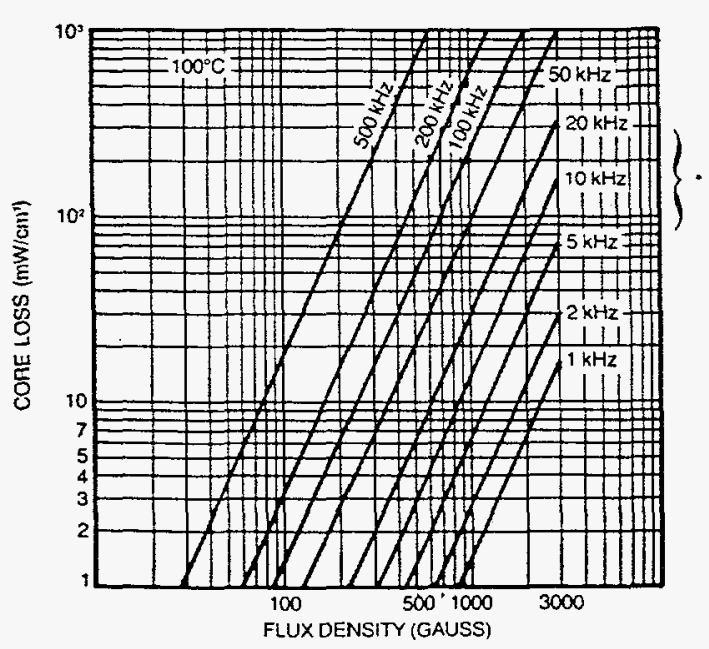

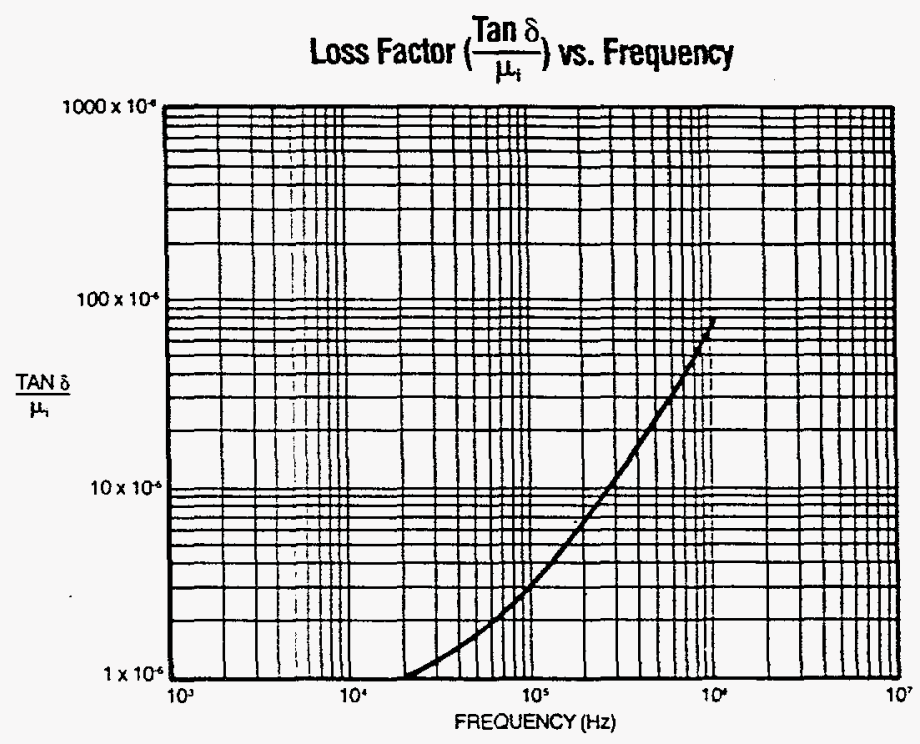

Pulse Characteristics
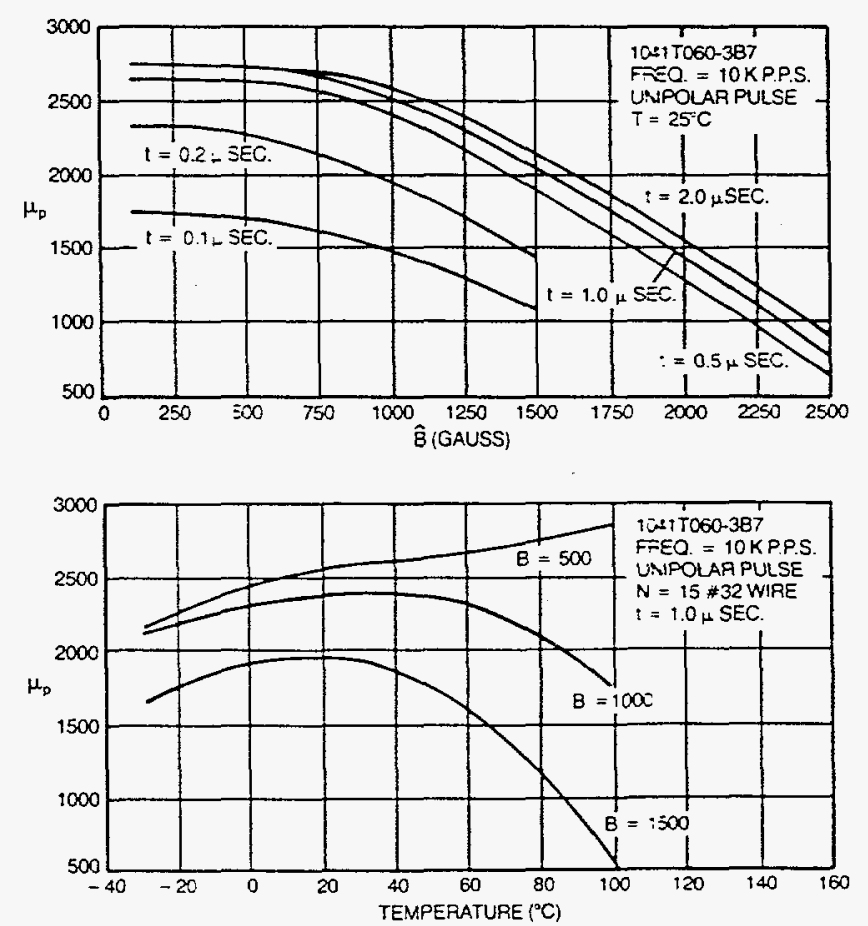

- Recommended operating range for toss limited designs. Flux density for bipolar excitation is $\dot{B}$.

Flux density for unipolar excitation is $\frac{B p-p \text {. }}{2}$

Figure 9. Data sheets on 704 pot core transformer (from [15]). b) Magnetic properties of ferrite materials. Sheet 2 of 2 . 
outer region of the core which bypasses the windings. Ignoring the flux in the region occupied by the windings themselves we have

$$
\Phi \approx \Phi_{b}+\Phi_{w}
$$

Ignoring the top and bottom paths and equating the $m m f$ for each vertical path gives

$$
R_{b} \Phi_{b}=\left(R_{w}+R_{g}\right) \Phi_{w}
$$

where the reluctances are

$$
\begin{gathered}
R_{b}=\frac{1}{\mu} \frac{0.164 \mathrm{in}}{\frac{1}{2}(0.285 \mathrm{in}-0.232 \mathrm{in})[\pi(0.285 \mathrm{in}+0.232 \mathrm{in})-2(0.071 \mathrm{in})](25.4 \mathrm{~mm} / \mathrm{in})} \approx \frac{1}{\mu(6.083 \mathrm{~mm})} \\
R_{w}=\frac{1}{\mu} \frac{0.164 \mathrm{in}}{\frac{1}{2}(0.115 \mathrm{in}-0.056 \mathrm{in}) \pi(0.115 \mathrm{in}+0.056 \mathrm{in})(25.4 \mathrm{~mm} / \mathrm{in})} \approx \frac{1}{\mu(2.454 \mathrm{~mm})} \\
R_{g}=\frac{1}{\mu_{0}} \frac{0.0024 \mathrm{in}}{\frac{1}{2}(0.115 \mathrm{in}-0.056 \mathrm{in}) \pi(0.115 \mathrm{in}+0.056 \mathrm{in})(25.4 \mathrm{~mm} / \mathrm{in})} \approx \frac{1}{\mu_{0}(167.7 \mathrm{~mm})}
\end{gathered}
$$

and the reluctance $R_{g}$ results from the air gap. We have assumed the air gap to be $0.0024 \mathrm{in}$, the remaining dimensions are taken from Figure 9. Taking the initial permeability of the material from Figure 9 to be

$$
\mu \approx 2300 \mu_{0}
$$

gives

$$
\Phi_{w} / \Phi \approx \frac{R_{b}}{R_{w}+R_{g}+R_{b}} \approx 0.0115
$$

Thus at low field levels all but about $1 \%$ of the flux is diverted around the windings. The induced output winding voltage will be

$$
\begin{gathered}
V \approx N \frac{\partial}{\partial t} \Phi_{w} \approx N\left(\frac{R_{b}}{R_{w}+R_{g}+R_{b}}\right)\left(\frac{\Phi}{\pi b_{t}^{2} \mu_{0} H_{i n}}\right) \pi b_{t}^{2} \mu_{0} \frac{\partial}{\partial t} H_{i n}^{s t e p} \\
\approx 400(0.0115)(2.0423)\left(3.85 \times 10^{-5} \mathrm{~m}^{2}\right) \mu_{0} \frac{\partial}{\partial t} H_{i n}^{\text {step }} \approx \begin{array}{c}
236 \text { volts (stainless steel) } \\
13.2 \text { volts (commercial aluminum) }
\end{array}
\end{gathered}
$$

where $N=400$ is taken as the number of output winding turns and (15) is used for $\frac{\partial}{\partial t} H_{i n}^{\text {step }}$. This output voltage is below threshold.

Unfortunately nonlinearity will reduce the effectiveness of the air gap in diverting the flux around the center of the pot core. To obtain a feel for how important saturation is, we note from Figure 9 that the saturation flux $B_{s}=0.38 \mathrm{~T}$ ( 3800 gauss) is achieved at a magnetic field intensity of $H_{c} \approx 160 \mathrm{~A} / \mathrm{m}$ (2 oersted) (which is typical of ferrite materials [17]). (Of course a much larger magnetic field is usually required to drive the relative magnetic permeability to unity.) The exterior exciting field has a peak value $H_{0} \approx 2.2 \mathrm{MA} / \mathrm{m}\left(\mu_{0} H_{0} \approx 2.8 \mathrm{~T}\right)$. Approximate (for early time an 
asymptotic result was obtained from integration of the first expression in (9), for late time the second expression in (9) was approximated by its first term and integrated) integration of the delta function interior field response (9) gives

$$
\begin{gathered}
H_{i n}^{s t e p}(t) \approx H_{0} \frac{8}{\xi \sqrt{\pi}}\left(\frac{t}{\tau_{d}}\right)^{3 / 2} e^{-\tau_{d} /(4 t)}, t / \tau_{d}<0.1 \\
H_{i n}^{\text {step }}(t) \approx H_{0}\left[1-e^{-t /\left(\xi \tau_{d}\right)}\right], t / \tau_{d}>>0.1
\end{gathered}
$$

We see that the interior magnetic field approaches the exterior magnetic field with time constant $\xi \tau_{d}$, which is small compared to the worst case lightning fall time (particularly for stainless steel). (Figure 7 shows that for the nearby filament source, the magnetic field also achieves large values in the fall time interval of the pulse.) Thus the transformer will be driven well into saturation and the magnetic permeability $\mu \rightarrow \mu_{0}$. The air gap is thus no longer effective in diverting the flux around the center core. In fact if $\mu=100 \mu_{0}$ the ratio in (69) is equal to 0.14 and if $\mu=10 \mu_{0}$ the ratio in (69) is equal to 0.26 .

Because of saturation it is worthwhile to consider the case of an aircore (fully saturated core) with $N=400$ turns. The average effective (average area) winding radius from Figure 9 is $b_{w} \approx 2.3$ $\mathrm{mm}$. Thus, using (15), we find

$$
V \approx N \pi b_{w}^{2} \mu_{0} \frac{\partial}{\partial t} H_{i n}^{s t e p} \approx \begin{gathered}
4.3 \mathrm{kV} \text { (stainless steel) } \\
242 \text { volts (commercial aluminum) }
\end{gathered}
$$

The stainless steel open circuit voltage is above threshold.

We could also inquire as to how large this might become if the lightning current is moved closer to the pill box than $\rho=b_{0}=14.5 \mathrm{~mm}$. Geometrical constraints require the line current to be above the top surface of the pill box; thus the realistic minimum height above the center of the transformer is approximately $4 \mathrm{~mm}$ ( $1 \mathrm{~mm}$ lightning conductor radius, $1 \mathrm{~mm}$ wall thickness, 2 $\mathrm{mm}$ transformer height). If the wire is centered above the transformer, symmetry and dominant horizontal orientation of the magnetic field (treating the top surface of the pill box as an infinite plane at early time where the maximum time derivative occurs) would reduce the transformer flux. Thus some horizontal displacement of the lightning conductor would be necessary to provide the appropriate component of magnetic field at the transformer. A horizontal displacement of 4 $\mathrm{mm}$ ( $\sim$ transformer radius) combined with the resulting 45 degree orientation of the field at the center of the transformer would give roughly a factor of 2 increase $\left(H_{0} \approx \frac{I_{0}}{4 \sqrt{2} \mathrm{~mm}} \frac{1}{\sqrt{2}}\right.$ compared to $\left.\frac{I_{0}}{14.5 \mathrm{~mm}}\right)$ in the vertical late time magnetic field amplitude over that of $(7)$; hence a factor of two increase in the time derivative of the field (15) and in the open circuit voltage (72) would be near the worst case. It is interesting to note that 2 times (72) is close to the transformer turns ratio $400 / 10=40$ times the induced loop voltage (48). An alternative calculation of this worst case field derivative is to use the thin sheet impedance solution for a step function line current above a conducting layer, discussed in the previous section. The time derivative of the vector potential is $\frac{\partial}{\partial t} A_{z}=\frac{1}{\pi} I_{0} Z_{s}\left(\rho_{0}+2 t Z_{s} / \mu_{0}\right) /\left[\left(\rho_{0}+2 t Z_{s} / \mu_{0}\right)^{2}+x^{2}\right]$, where $x$ is the horizontal shift of the line current with respect to the observation point below the layer. The derivative of the vertical magnetic field is thus $\frac{\partial}{\partial t} H_{y}=\frac{2}{\pi} I_{0} Z_{s} x\left(\rho_{0}+2 t Z_{s} / \mu_{0}\right) /\left[\left(\rho_{0}+2 t Z_{s} / \mu_{0}\right)^{2}+x^{2}\right]^{2}$. The maximum occurs at $x=\left(\rho_{0}+2 t Z_{s} / \mu_{0}\right) / \sqrt{3}$ and again at $t=0$. Thus we find the peak value from the impedance solution

$$
\frac{\partial}{\partial t} H_{y} \approx \frac{3 \sqrt{3}}{8 \pi} \frac{I_{0}}{\mu_{0} \sigma \Delta \rho_{0}^{2}} \approx 1.5 \times 10^{12} \mathrm{~A} / \mathrm{m} / \mathrm{sec}
$$


where we have set $\rho_{0} \approx 1 \mathrm{~mm}+1 \mathrm{~mm}+2 \mathrm{~mm}=4 \mathrm{~mm}$, the vertical spacing from the line source to the center of the transformer. This value is only $44 \%$ bigger than twice the time derivative (15) (the value previously proposed for the worst case). The result (73) is an overestimate since the bottom conducting wall of the pill box tends to reduce the normal field component (furthermore it is thought that the impedance result at time $t=0$ overestimates the field derivative).

More detailed modeling of the transformer (for example, quantitative modeling of the nonlinearity, impedance loading, and leakage flux) and interior circuits (for example, more realistic loop estimates of the circuit traces) could possibly reduce the capacitor charge voltage below threshold). These considerations are out of the scope of this report. The following simple example is as far as we take the transformer modeling.

It is instructive to consider the case of a shorted primary load on the transformer. The primary winding is typically inside the secondary winding as indicated by the large dots in Figure 8 . The shorted primary load prevents magnetic flux from coupling to the center ferrite region. (We expect the outer shell of the transformer to rapidly saturate.) However, the free space area of the secondary winding is not much smaller than the total area and thus the open circuit voltage (72) is still appropriate. The free space area of the secondary winding also creates a leakage inductance, which the open circuit voltage (72) appears in series with. If we very roughly approximate this leakage inductance with the inductance of a solenoid in free space [18] we obtain

$$
L_{w} \approx K\left(2 b_{w} / h_{s}\right) N^{2} \mu_{0} \pi b_{w}^{2} / h_{s} \approx 0.67 \mathrm{mH}
$$

where $h_{s} \approx 2.9 \mathrm{~mm}$ is the wire coil height and $K(1.586) \approx 0.581$ [18]. Figure 10 shows the resulting secondary circuit with the open circuit voltage $V_{o c} \approx V_{0} e^{-t /\left(\xi \tau_{d}\right)}$ and amplitude $V_{0}$ given by (72). One quarter period of the output circuit response is

$$
t_{1 / 4}=\frac{\pi}{2} \sqrt{L_{w} C_{\text {out }}} \approx 11.1 \mu \mathrm{sec}
$$

Since, for stainless steel, this time is much larger than the decay time $\xi \tau_{d} \approx 2.8 \mu$ sec, we can approximate the inductor current at early time as

$$
I \approx \frac{1}{L_{w}} \int_{0}^{t} V_{o c} d t \approx V_{0} \xi \tau_{d} / L_{w}, t>>\xi \tau_{d}
$$

The energy which will eventually be transferred to the capacitor is thus

$$
W_{\text {out }}=\frac{1}{2} L_{w} I^{2} \approx V_{0}^{2} \xi^{2} \tau_{d}^{2} /\left(2 L_{w}\right) \approx 107 \mathrm{~mJ}
$$

This value is nearly a factor of six above the threshold charge energy. Note that, if a more realistic primary load was attached to the transformer, the resulting placement of that load in the secondary (multiplied by the square of the turns ratio $40^{2}$ ) could substantially reduce this energy.

More accurate detailed calculations of the interior circuit response to the interior stimulus provided in this report are out of scope of the present analysis.

\section{TWO DIMENSIONAL FINITE ELEMENT MODEL}

A two dimensional finite element code was used to model the pill box barrier under plane field excitation. To further reduce the complexity of the model only one half the structure was modeled as shown in Figure 11. (The placement of the foil region, whether flush with the interior wall surface or flush with the exterior wall surface is not critical to the calculations.) The transformer was approximately modeled as a PMC structure for large permeability or alternatively as an air structure $\mu_{0}$ when fully saturated. (The symmetry plane at $x=0$ thus introduced an imaged 


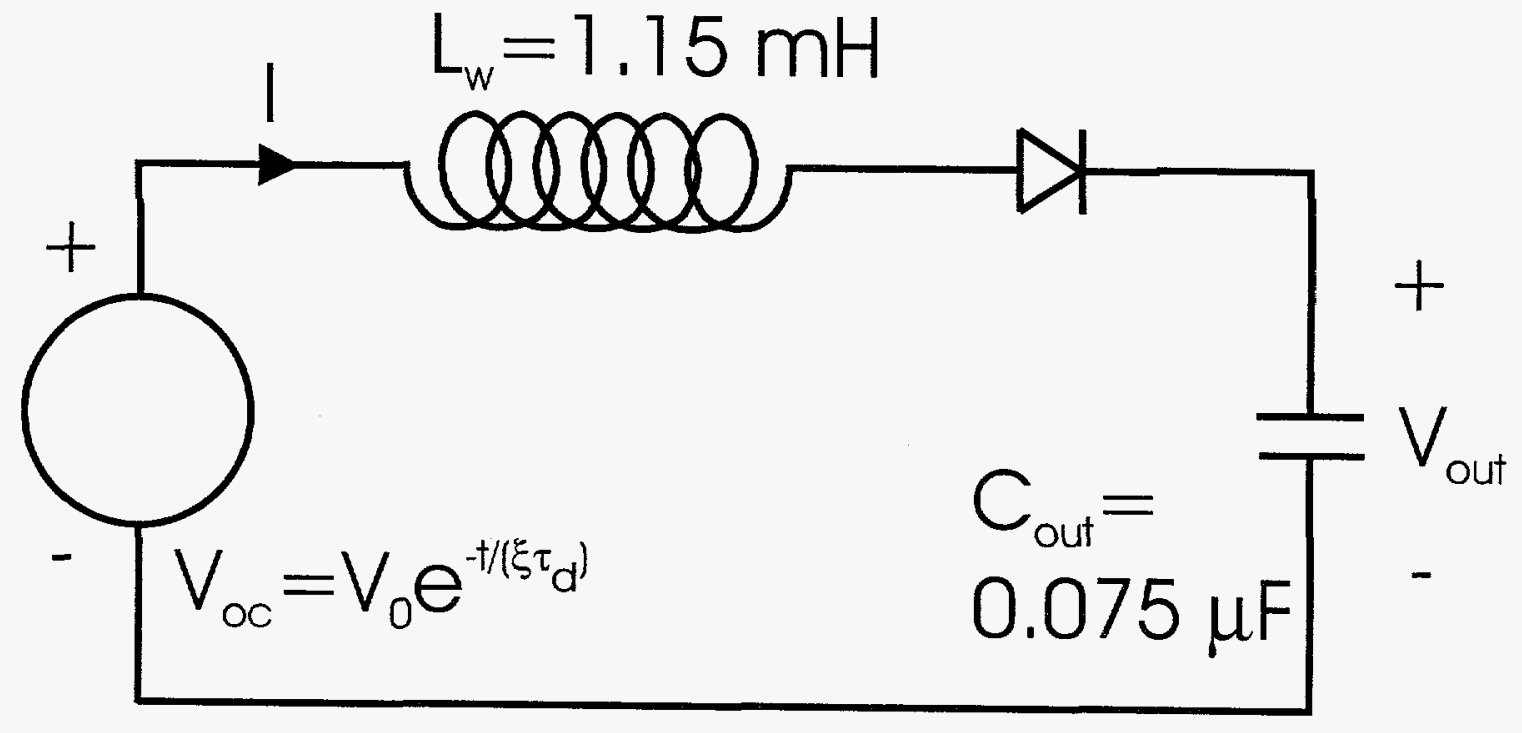

Figure 10. Output capacitor charging circuit for direct magnetic field coupling to transformer secondary (with primary circuit shorted).

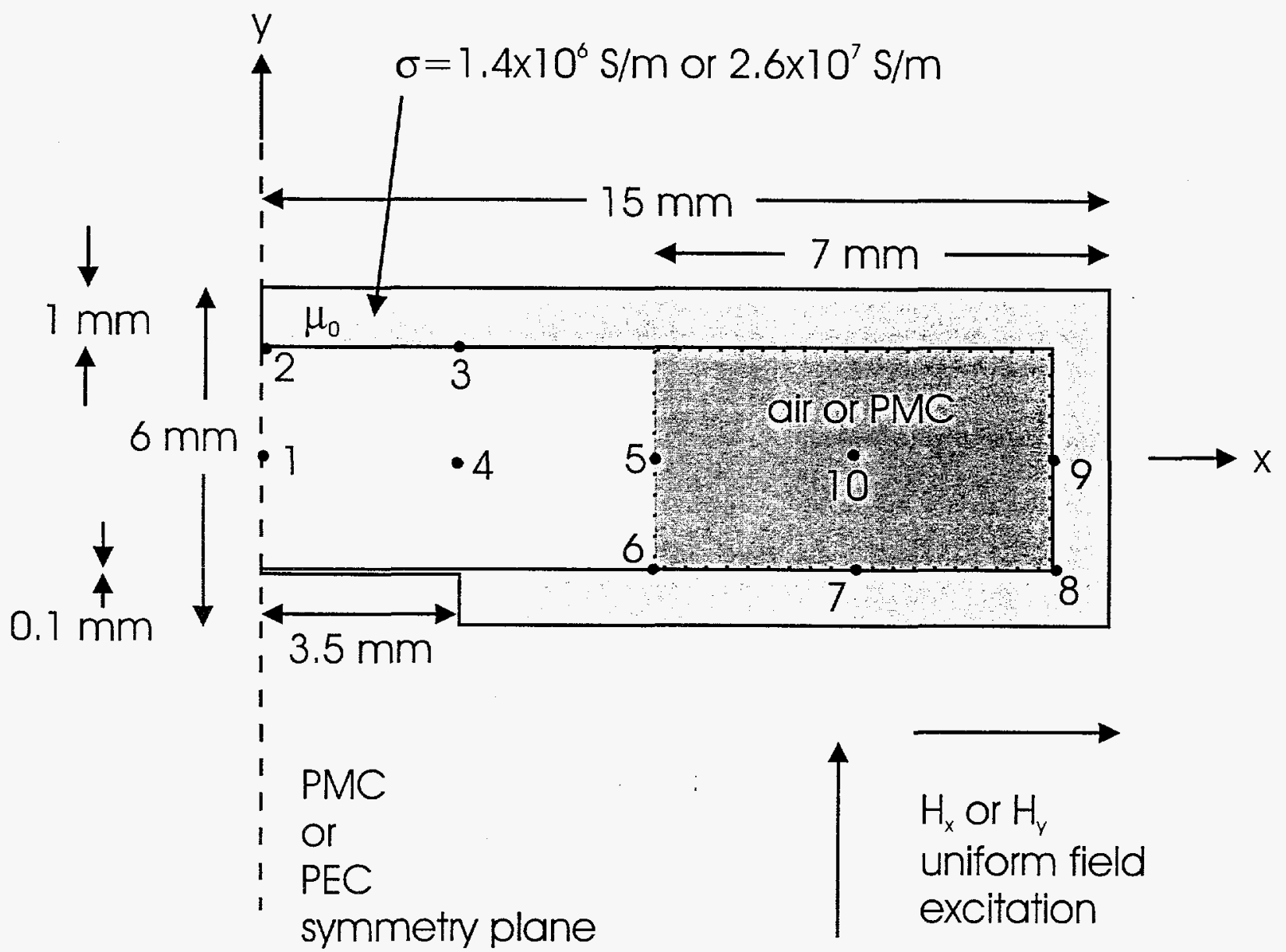

Figure 11. Two dimensional finite element model geometry. Numerical field evaluation points $1-10$ are shown as black dots. 
PMC about $x=0$. This was far enough removed from the problem that it was felt it would not significantly perturb the fields.) Figure 12 shows the finite element mesh used to discretize the problems. The code solved for the axial component of the vector potential $A_{z}$, the problem was thus scalar. The approximate incident plane uniform field $H_{x}$ or $H_{y}$ was excited by introducing a linear ramp of vector potential values along the appropriate part of the outer boundary of the grid (which was $45 \mathrm{~mm}$ on a side) with the convenient value of $1 \mathrm{MA} / \mathrm{m}$. The inverse exponential waveform (4) was used as the time modulation. Ten points are shown as black dots in Figure 11 at which the fields were determined from the code. The two dimensional approximation introduces several errors in the modeling of the three dimensional structure among which are: although the magnitude of the field near the foil will be approximately represented, the exponential decay of the $H_{x}$ field for large $x$ is not representative of the $1 / x^{2}$ line dipole behavior noted in the previous section on the foil penetration (note that $H_{y}$ here is equivalent to $H_{z}$ discussed previously), corner enhancements of the transformer magnetic flux are far larger here where corners of the PMC are adjacent to the corners of the shield (in the cylindrical transformer they are only adjacent at one point along the circumference).

Figures 13 through 16 show the magnetic field as a function of time in the aluminum and stainless steel pill box housings for both air and PMC cores (note that the field at the points 6 and 8 are not meaningful for the PMC core, since edge singularities exist and the finite values result from the finite mesh size). These results show that for the aluminum pill box shield and air core, the interior field parallel to the exciting field reaches 0.5 to 0.7 of the exterior field at peak (it does not reach unity because of the fall time of the incident field). The cross polarized field is relatively small (one exception is the corner field $H_{x}$ at point 8 for $y$ polarization of the incident field). The results for the stainless steel pill box shield and air core reach interior field levels, parallel to the exciting field, between 0.9 to 1 of the exterior field level. Again the cross polarized field is relatively small (one exception is again the corner field $H_{x}$ at point 8 for $y$ polarization of the incident field).

Figures 17 through 20 show the corresponding time derivatives of the interior field. The aluminum housing air core results for $x$ polarized incident field are the largest for the foil penetration (points 1,2,3, and 4) and the corner (point 8). The largest time derivative for the $y$ polarization is the corner field (point 8). If we ignore test points $1,2,3,4$, and 8 we see that $1.4 \times 10^{10} \mathrm{~A} / \mathrm{m} / \mathrm{sec}$ is a good estimate of the remaining curves with the exception of point 9 which has a value $3.2 \times 10^{10}$ $\mathrm{A} / \mathrm{m} / \mathrm{sec}$. Extrapolating the first value to the field level (7) (multiplying by 2.2 ) thus gives

$$
\frac{\partial}{\partial t} H_{\text {code }} \approx 1.4 \times 10^{10} \mathrm{~A} / \mathrm{m} / \mathrm{sec}(2.2) \approx 3.1 \times 10^{10} \mathrm{~A} / \mathrm{m} / \mathrm{sec}(\text { commercial aluminum) }
$$

which is quite close to the analytical estimate (15). Extrapolating point 9 to the field level (7) gives

$$
\frac{\partial}{\partial t} H_{\text {code }} \approx 3.3 \times 10^{10} \mathrm{~A} / \mathrm{m} / \mathrm{sec}(2.2) \approx 7.3 \times 10^{10} \mathrm{~A} / \mathrm{m} / \mathrm{sec} \text { (commercial aluminum) }
$$

which is slightly greater than twice the analytical estimate (15). This enhancement appears to be confined to the region near the outer boundary of the pill box since point 10 does not exhibit such an enhancement. The corner (point 8 ) total field derivative for the aluminum housing and air core is bounded by $5.9 \times 10^{10} \mathrm{~A} / \mathrm{m} / \mathrm{sec}$. This is four times the nominal interior value $\left(1.4 \times 10^{10} \mathrm{~A} / \mathrm{m} / \mathrm{sec}\right)$; thus the crude factor of two corner enhancement, previously estimated from Kaden's time harmonic results, somewhat underestimates the time domain corner enhancement (again this enhancement is only visible at the corner point 8 ). Because there are no susceptible loops within the foil radius (a loop around the foil will not link significant high frequency flux since the net flux through the 
-1วبırq ฮิụฺonpuos

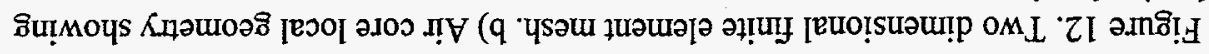

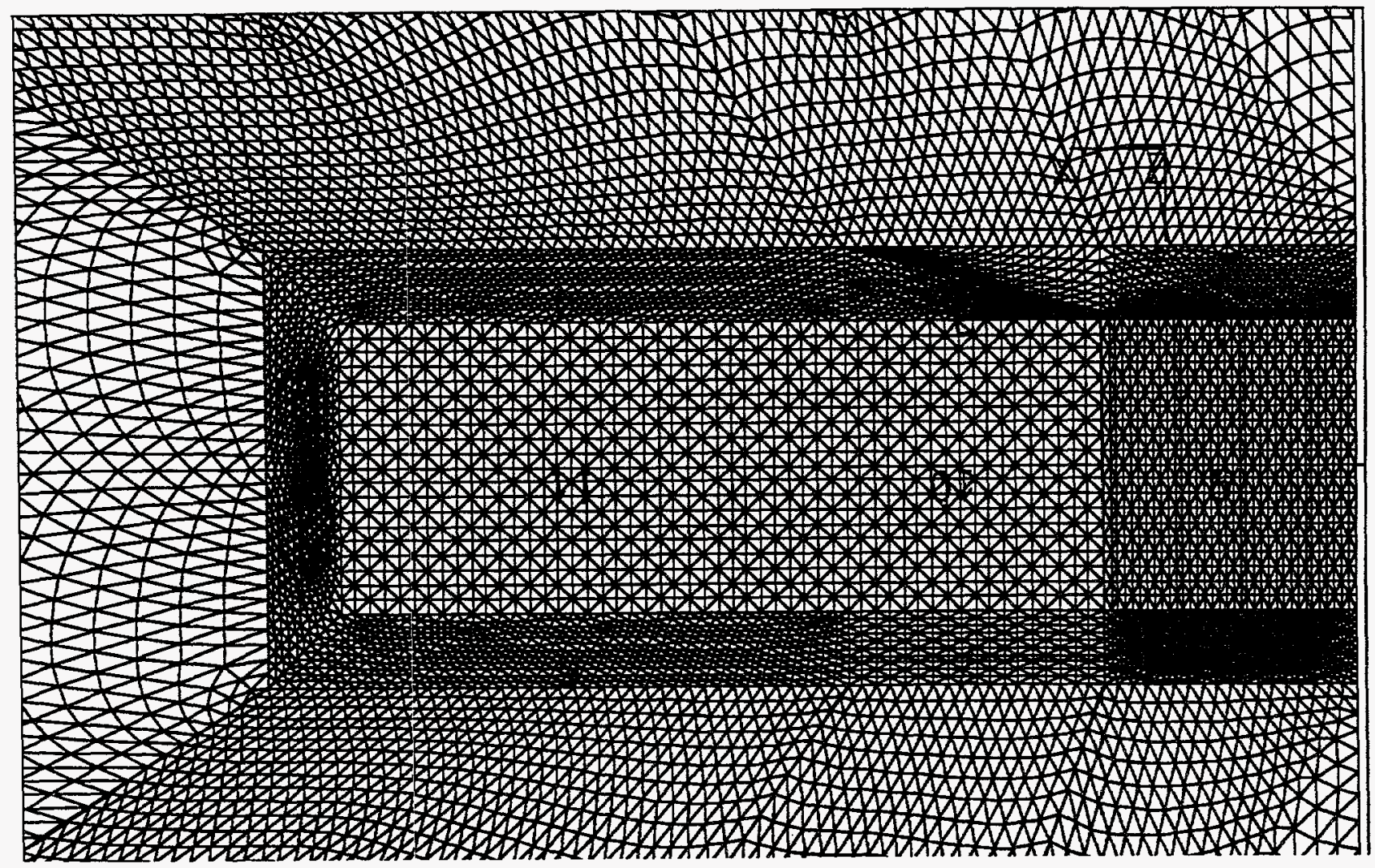

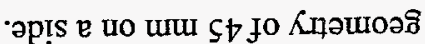

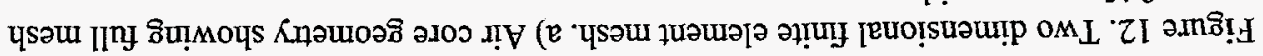

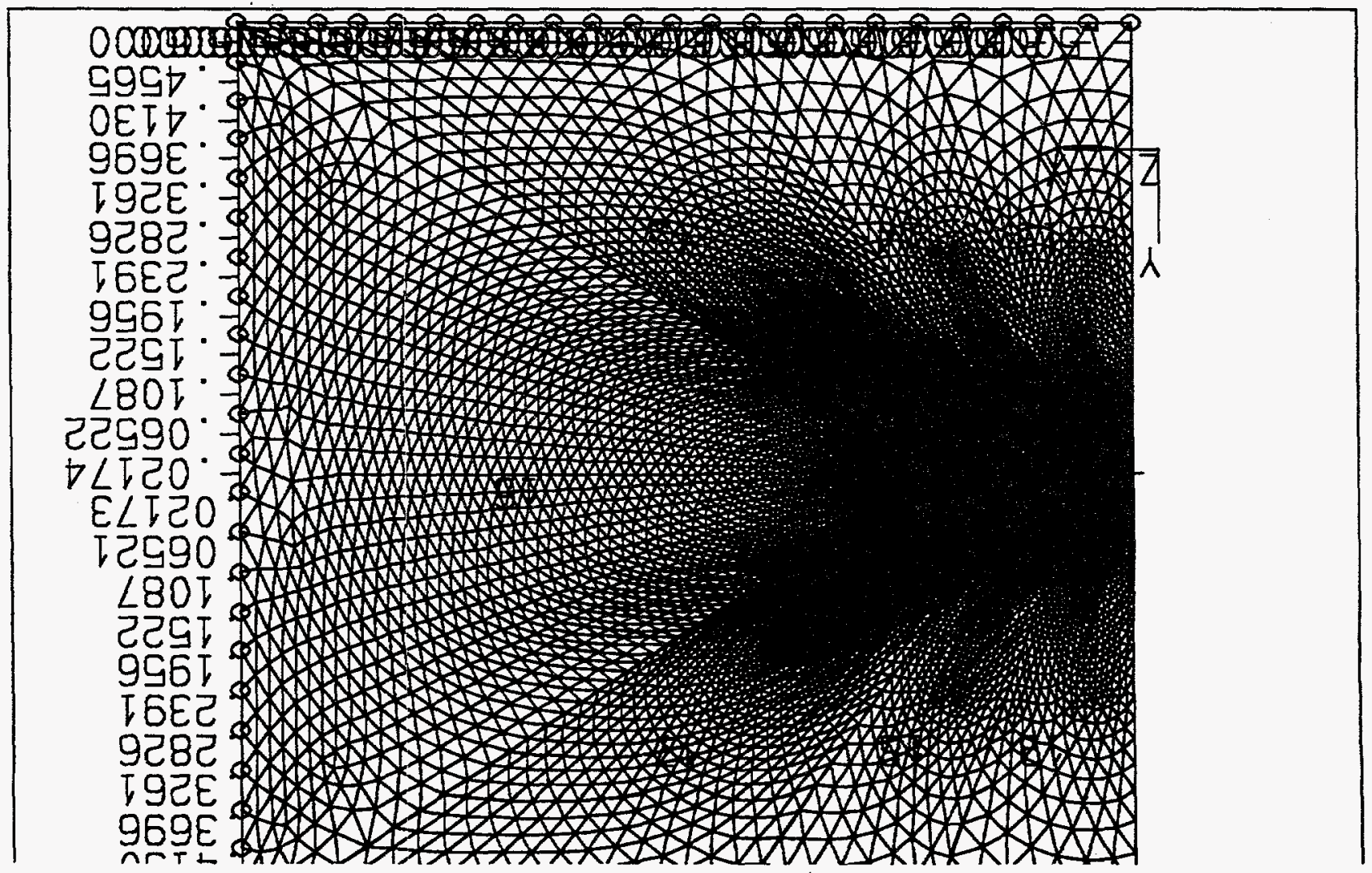




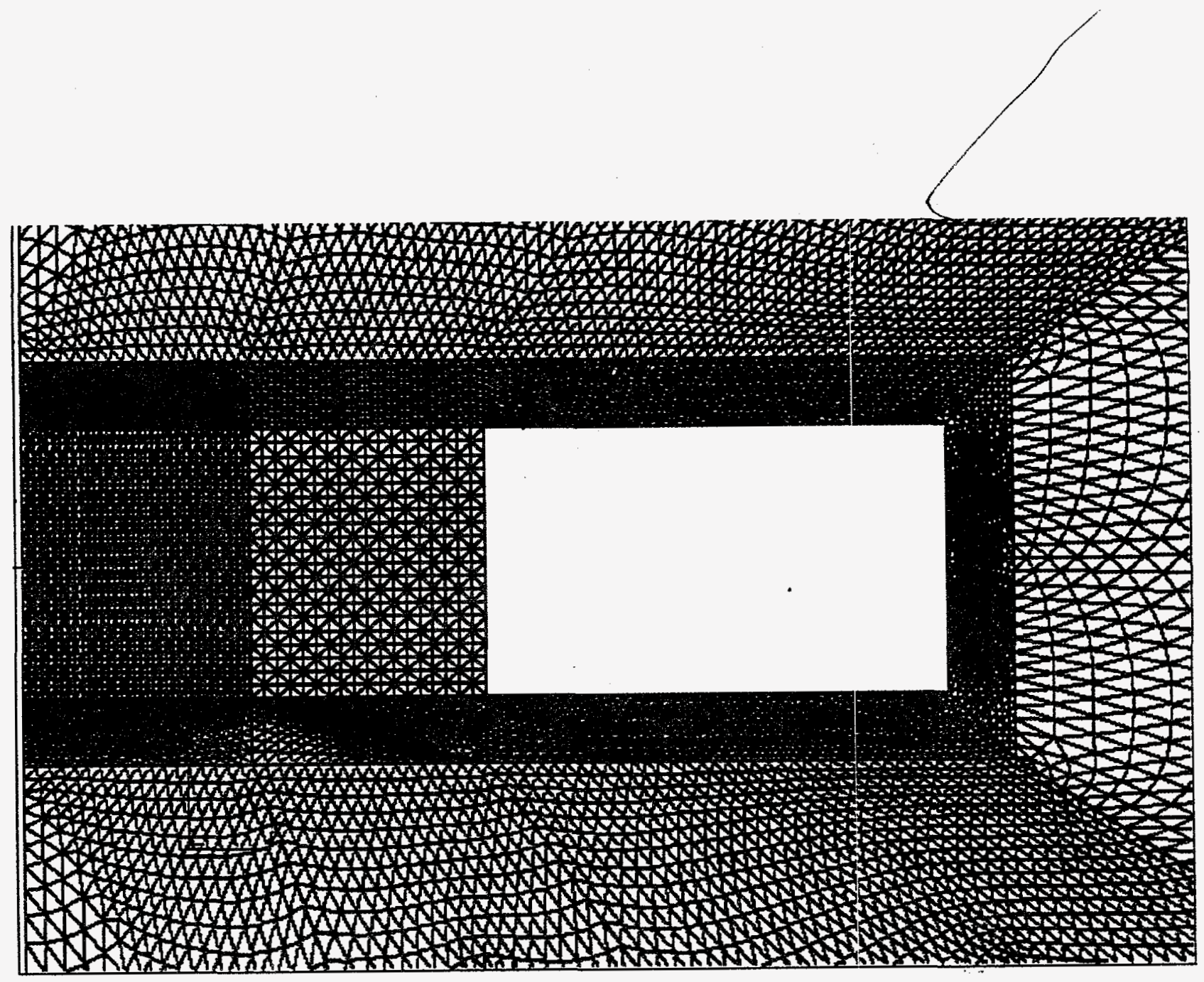

Figure 12. Two dimensional finite element mesh. c) PMC core local geometry showing conducting barrier and empty PMC core. 
Penetrant Magnetic Field

Aluminum Housing, Air Core, $\mathrm{Hinc}=\mathrm{Hx}$

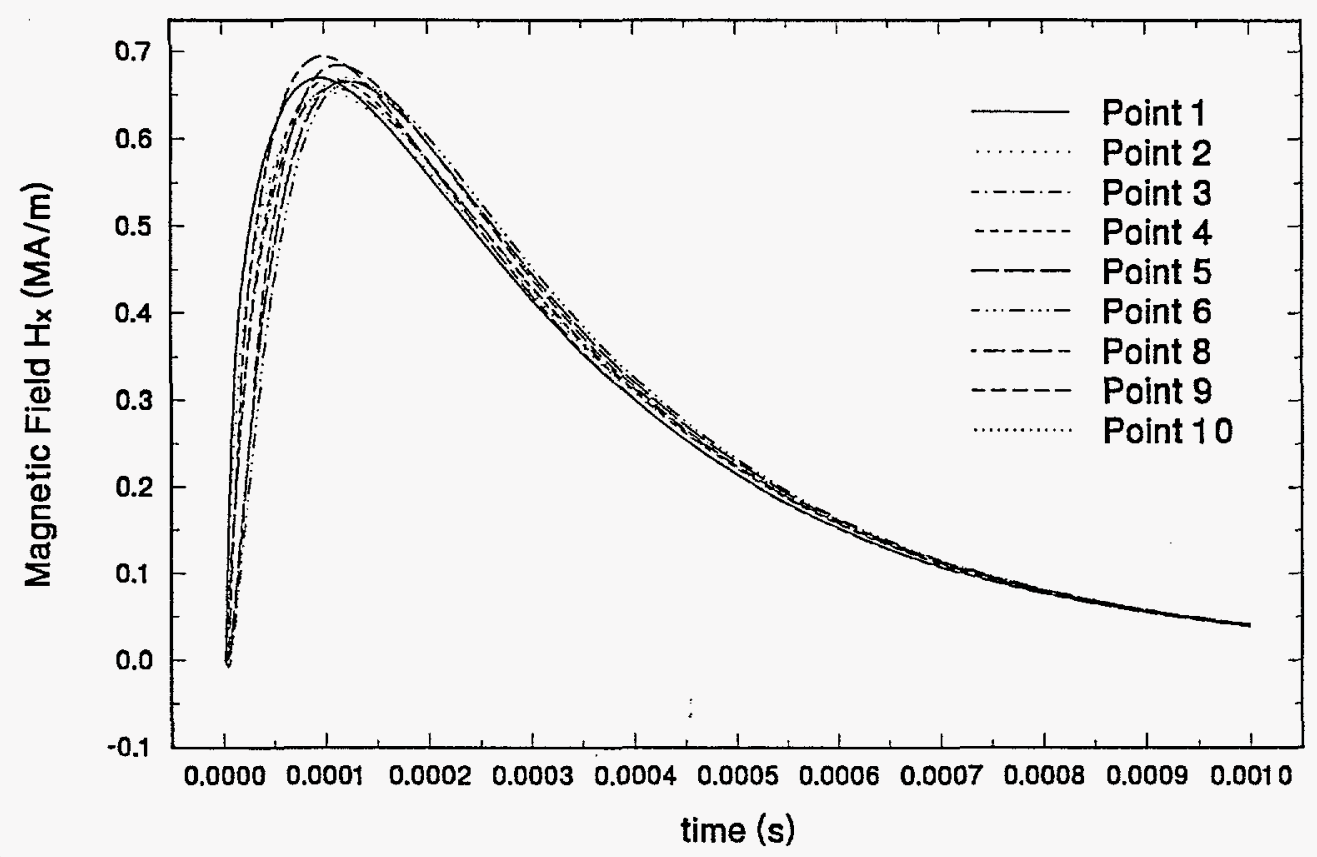

Penetrant Magnetic Field

Aluminum Housing, Air Core, $\mathrm{Hinc}^{\text {inc }} \mathrm{H}_{\mathrm{x}}$

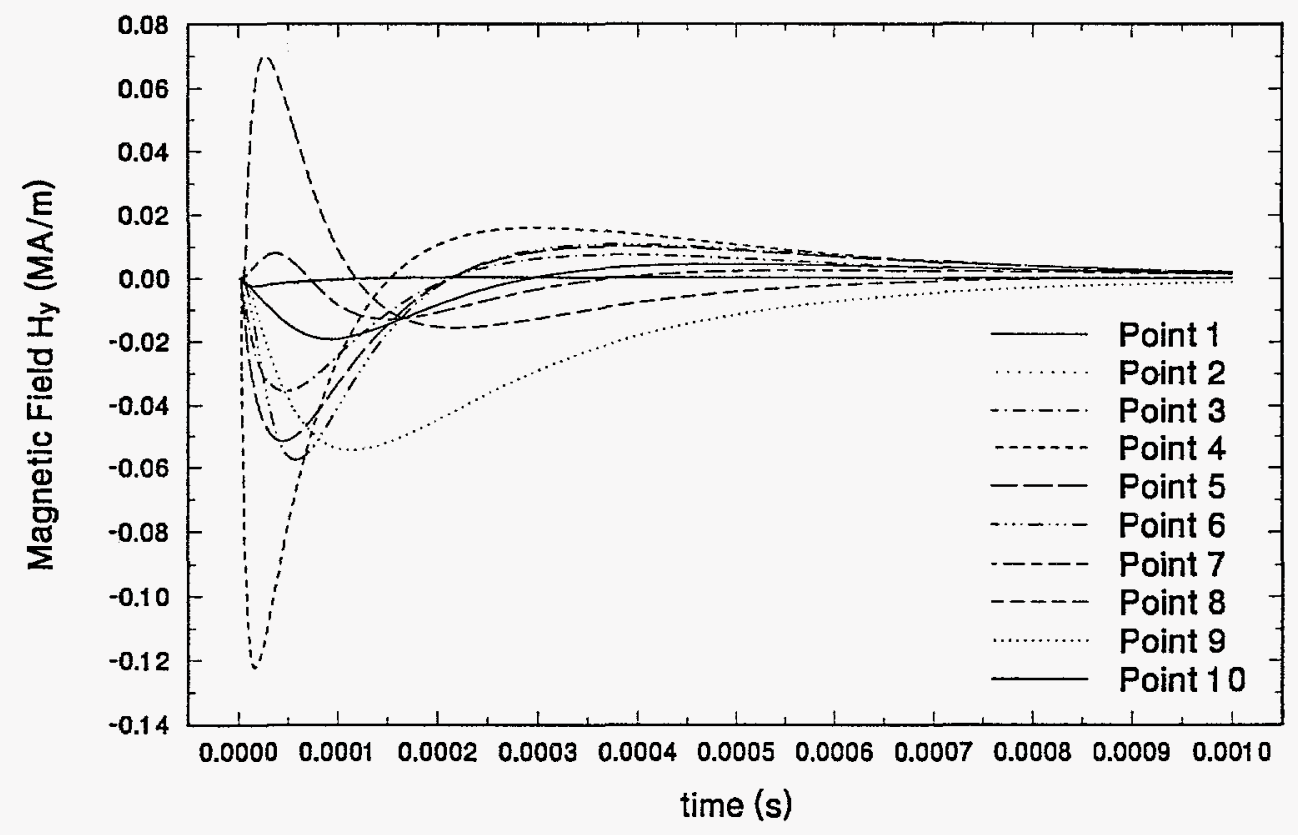

Figure 13. Finite element results for interior magnetic field (exterior uniform field is $1 \mathrm{MA} / \mathrm{m}$ ) with aluminum pill box barrier and air core. Graphs 1 and 2 of 4 . 


\section{Penetrant Magnetic Field}

Aluminum Housing, Air Core, $\mathrm{H}^{\text {inc }}=\mathrm{H}_{\mathrm{y}}$

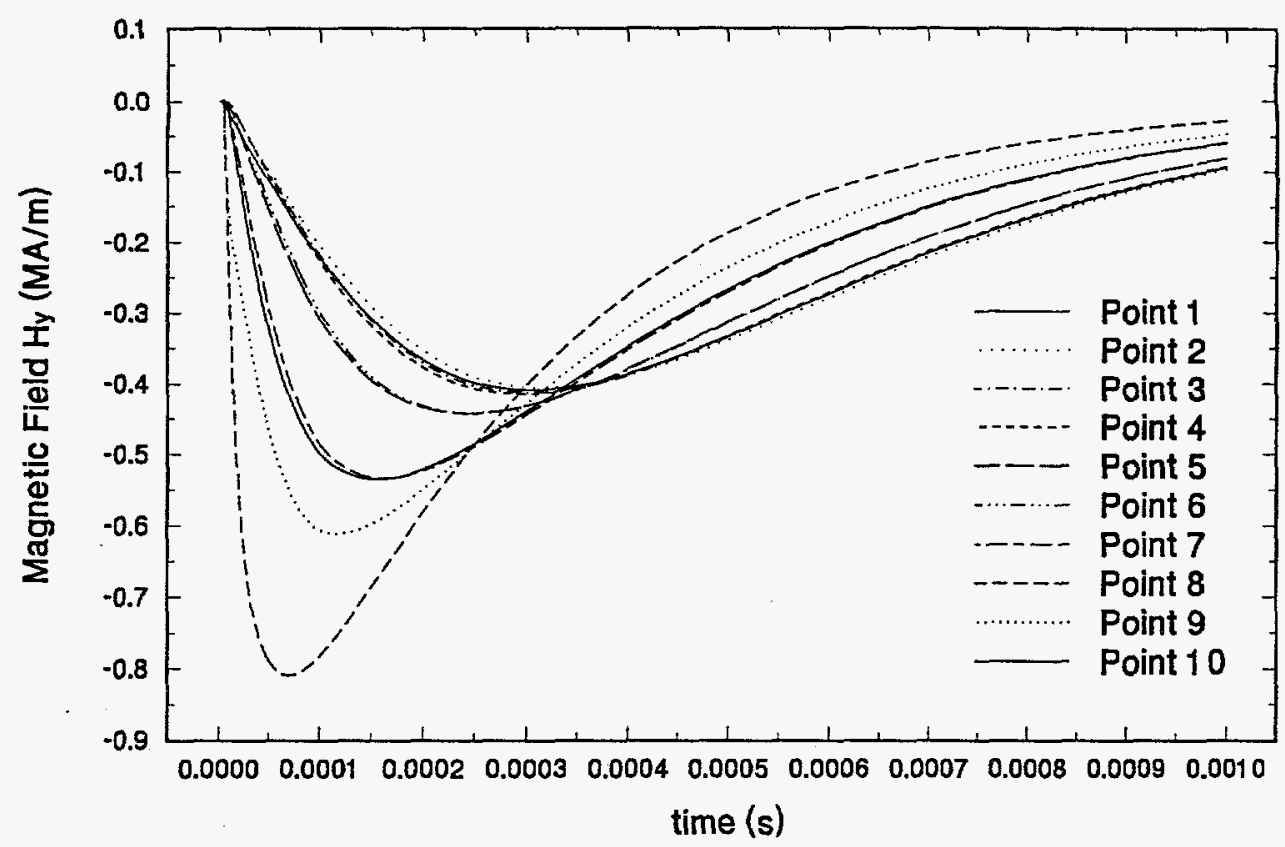

Penetrant Magnetic Field

Aluminum Housing, Air Core, $\mathrm{H}^{\text {inc }}=\mathrm{H}_{\mathrm{y}}$

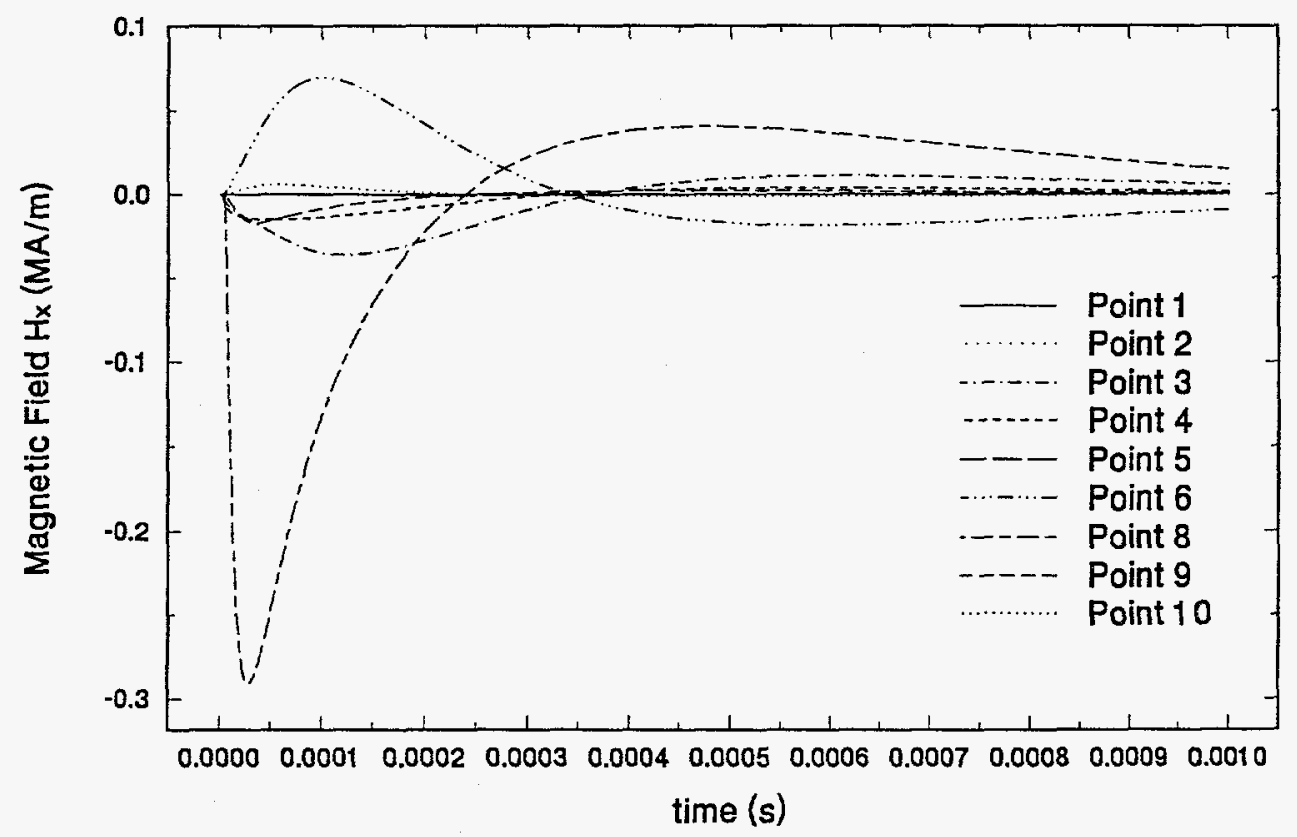

Figure 13. Finite element results for interior magnetic field (exterior uniform field is $1 \mathrm{MA} / \mathrm{m}$ ) with aluminum pill box barrier and air core. Graphs 3 and 4 of 4 . 
Penetrant Magnetic Field

Aluminum Housing, PMC Core, $\mathrm{H}^{\text {inc }}=\mathrm{H}_{\mathbf{x}}$

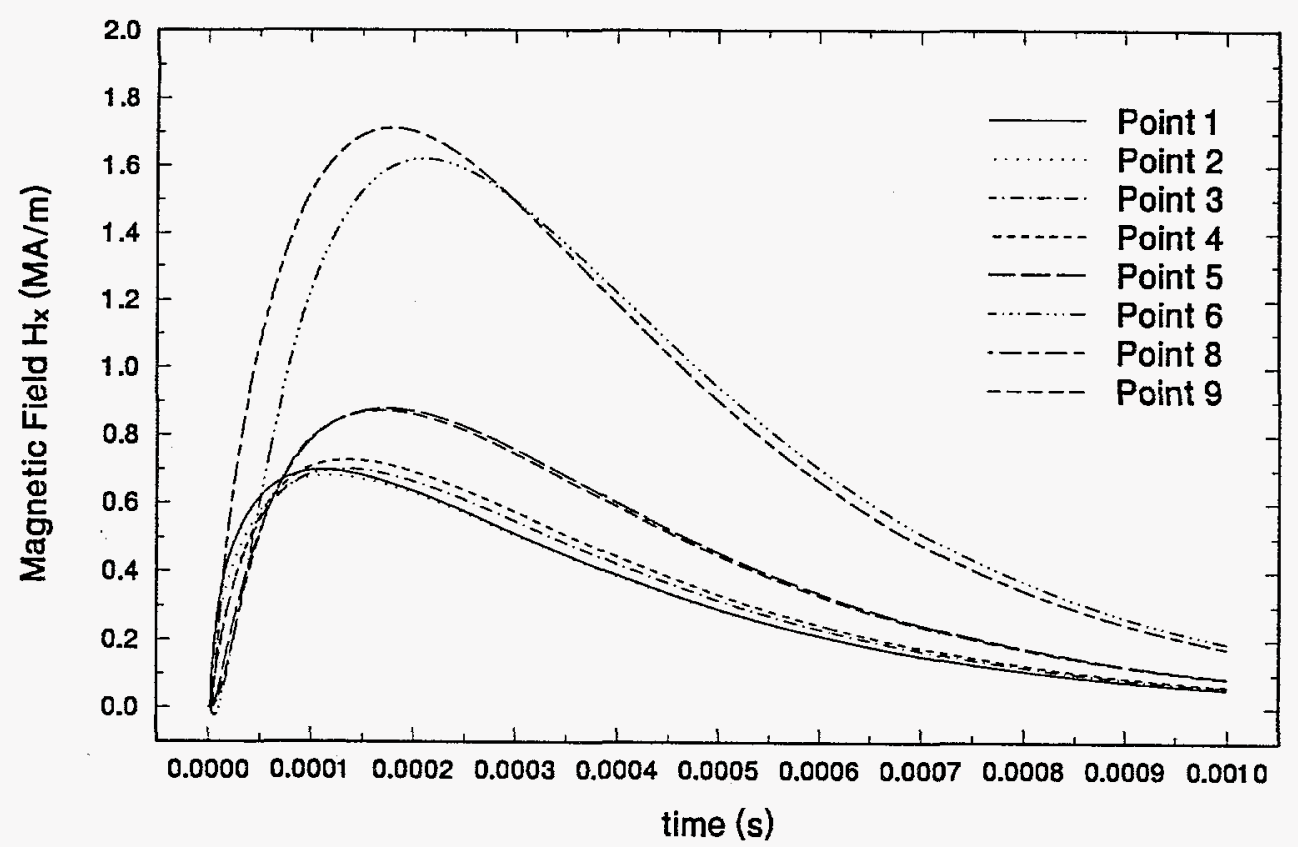

Penetrant Magnetic Field Aluminum Housing, PMC Core, $\mathrm{H}^{\text {inc }}=\mathrm{Hx}_{x}$

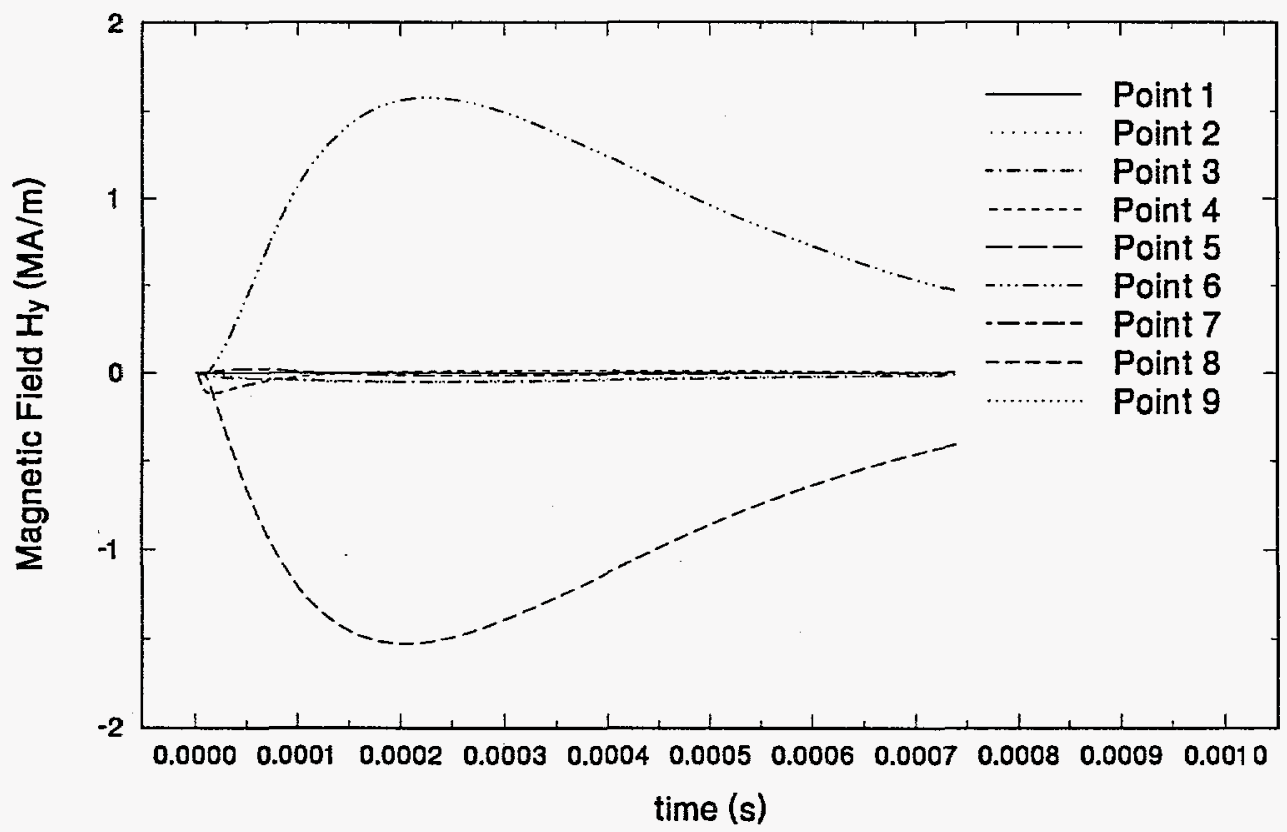

Figure 14. Finite element results for interior magnetic field (exterior uniform field is $1 \mathrm{MA} / \mathrm{m}$ ) with aluminum pill box barrier and PMC core. Graphs 1 and 2 of 4 . 
Penetrant Magnetic Field

Aluminum Housing, PMC Core, $\mathrm{H}^{\text {inc }}=\mathrm{Hy}$

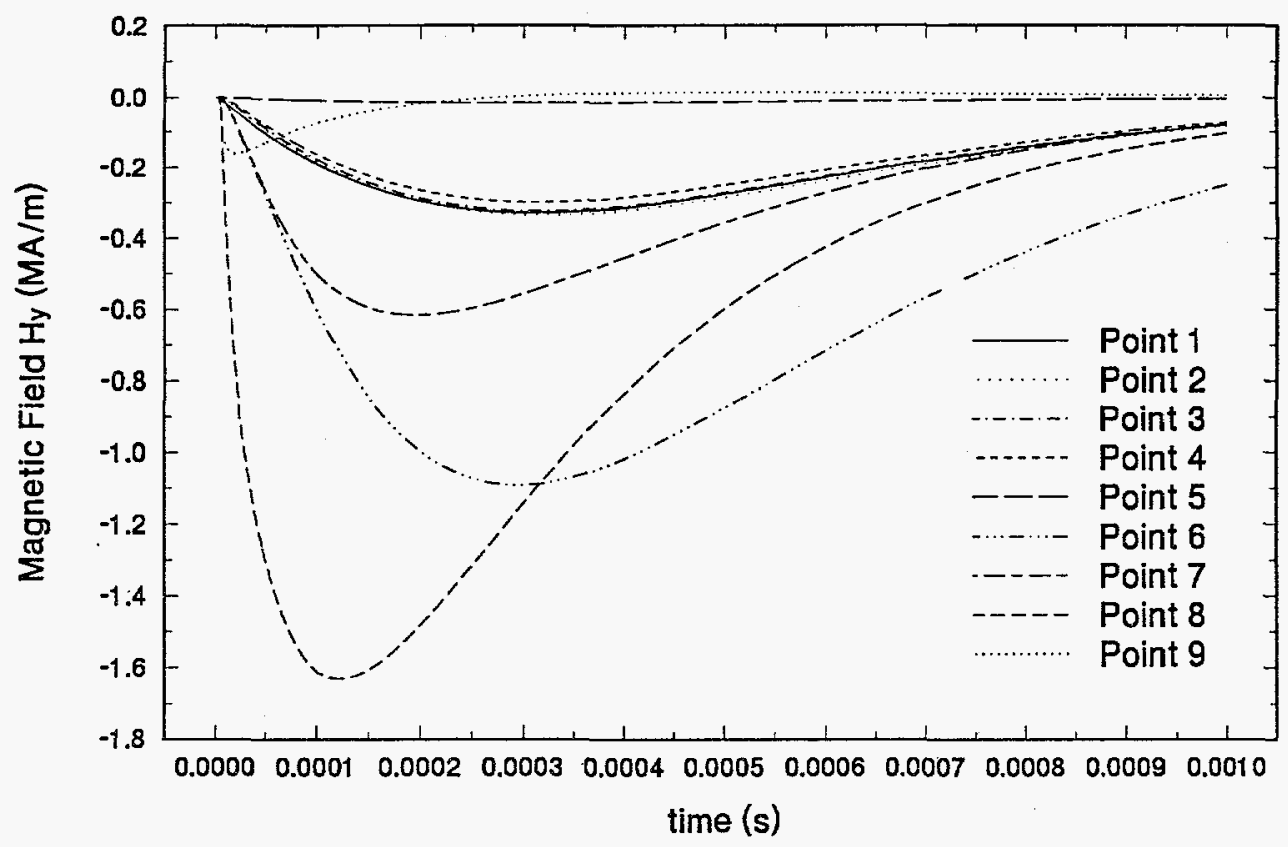

Penetrant Magnetic Field

Aluminum Housing, PMC Core, $\mathrm{H}^{\text {inc }}=\mathrm{H}_{\mathrm{y}}$

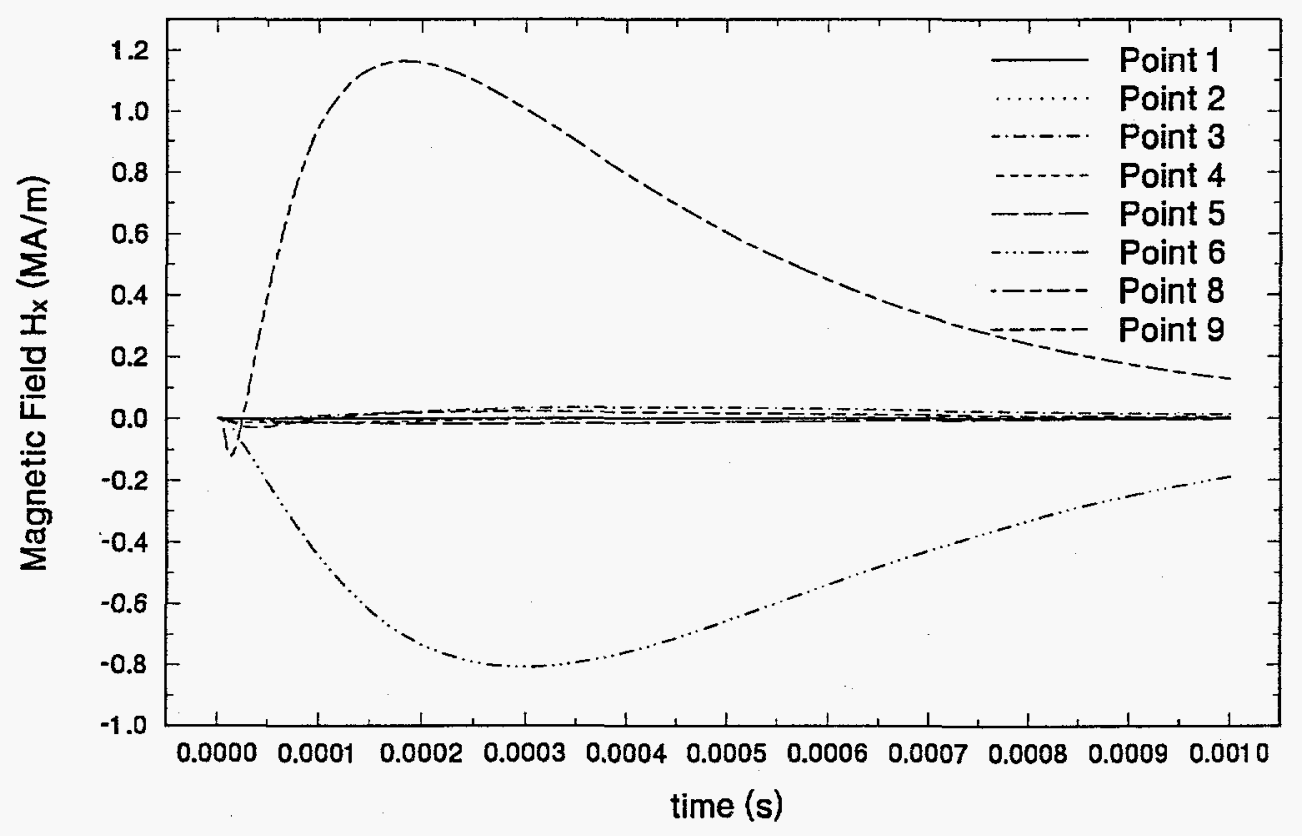

Figure 14. Finite element results for interior magnetic field (exterior uniform field is $1 \mathrm{MA} / \mathrm{m}$ ) with aluminum pill box barrier and PMC core. Graphs 3 and 4 of 4 . 
Penetrant Magnetic Field

\section{Stainless Steel Housing, Air Core, $\mathrm{Hinc}^{\mathrm{in}} \mathrm{Hx}$}

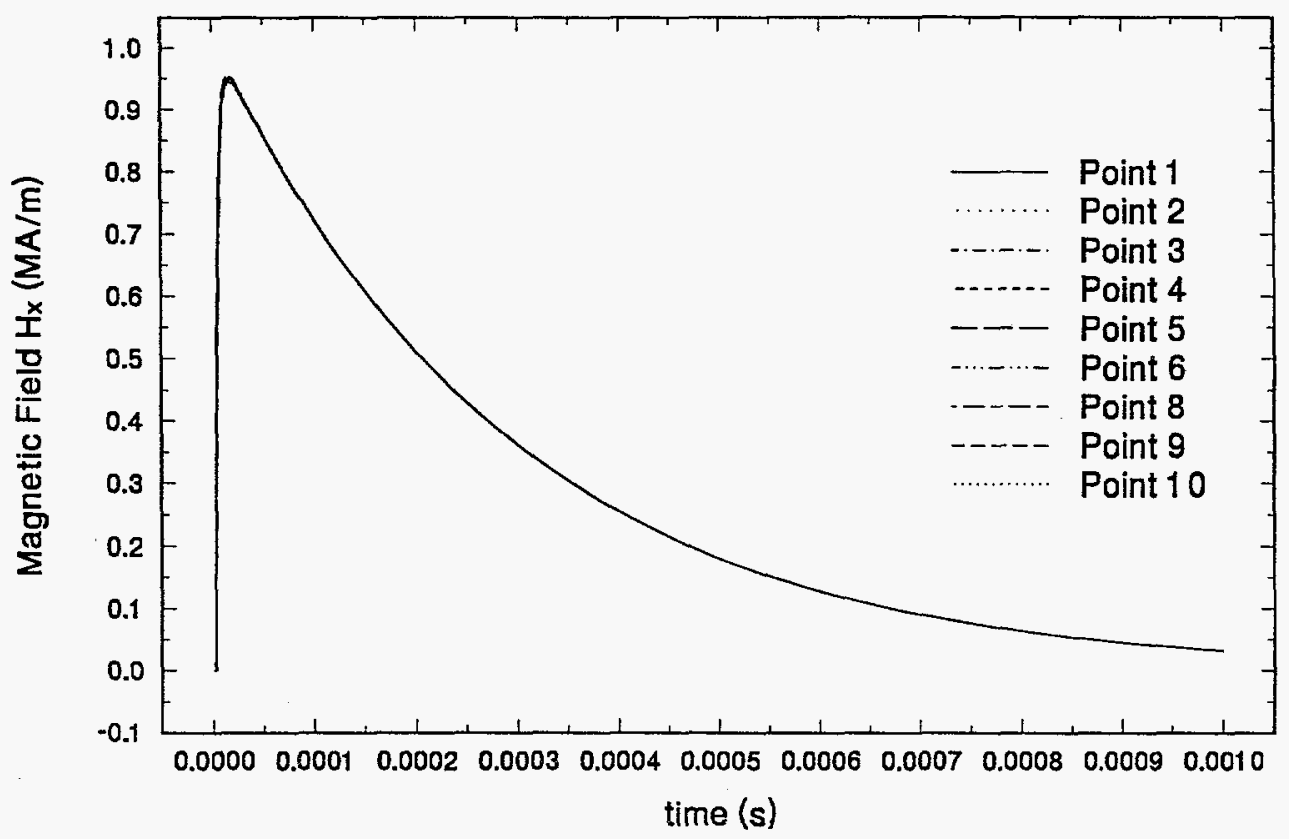

Penetrant Magnetic Field

Stainless Steel Housing, Air Core, $\mathrm{Hinc}^{\mathrm{in}}=\mathrm{Hx}_{\mathrm{x}}$

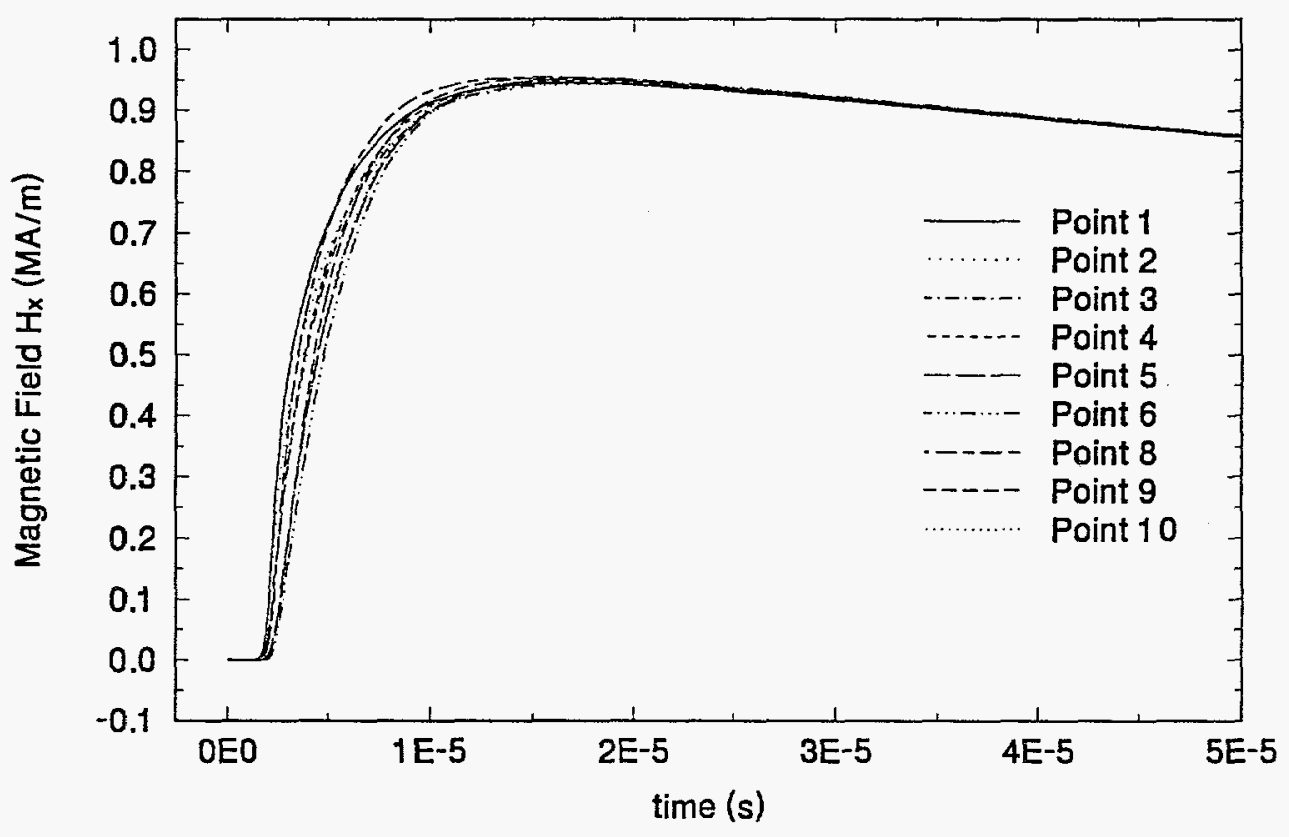

Figure 15. Finite element results for interior magnetic field (exterior uniform field is $1 \mathrm{MA} / \mathrm{m}$ ) with stainless steel pill box barrier and air core. Graphs 1 and 2 of 5 . 
Penetrant Magnetic Field

Stainless Steel Housing, Air Core, $\mathrm{H}^{\text {inc }}=\mathrm{Hx}_{\mathrm{x}}$

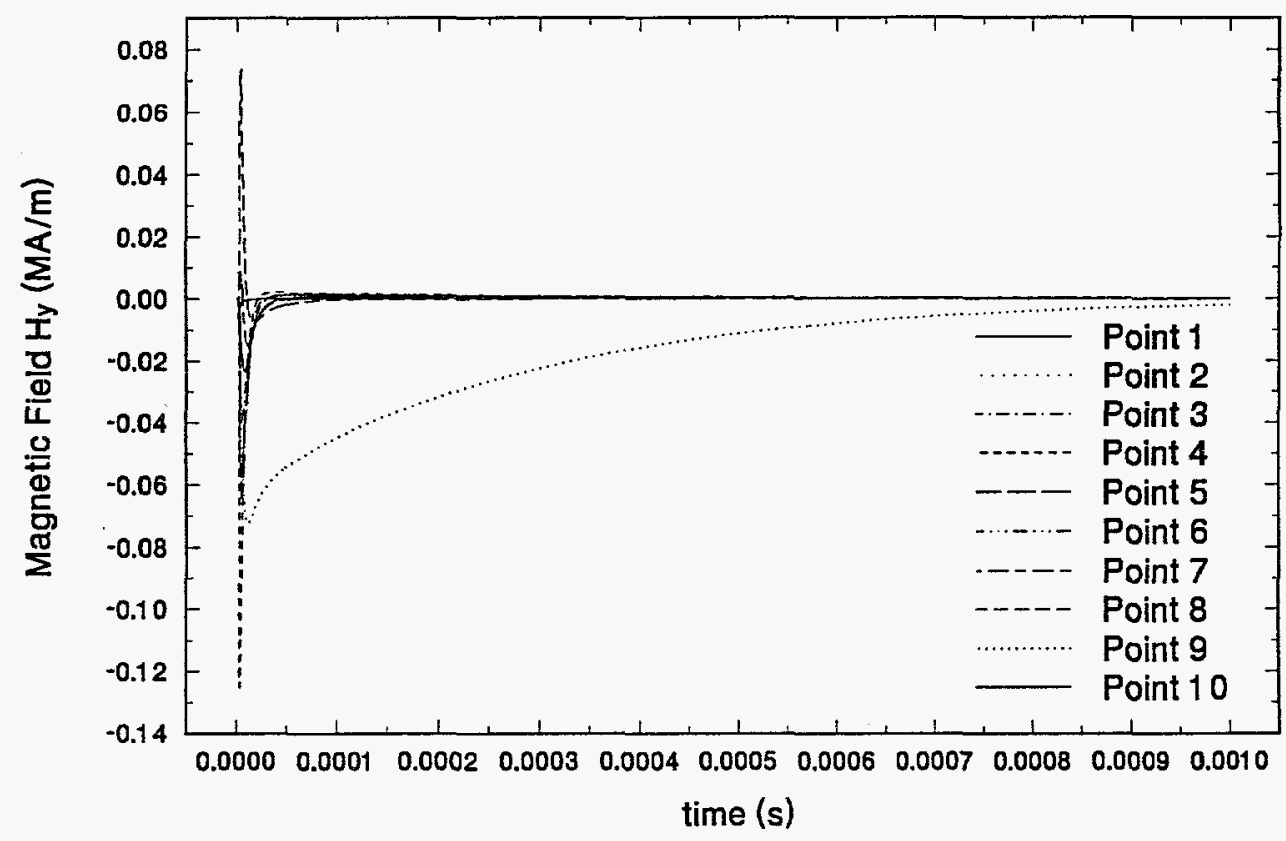

Penetrant Magnetic Field

Stainless Steel Housing, Air Core, $\mathrm{Hinc}^{\text {inc }} \mathrm{Hy}_{\mathrm{y}}$

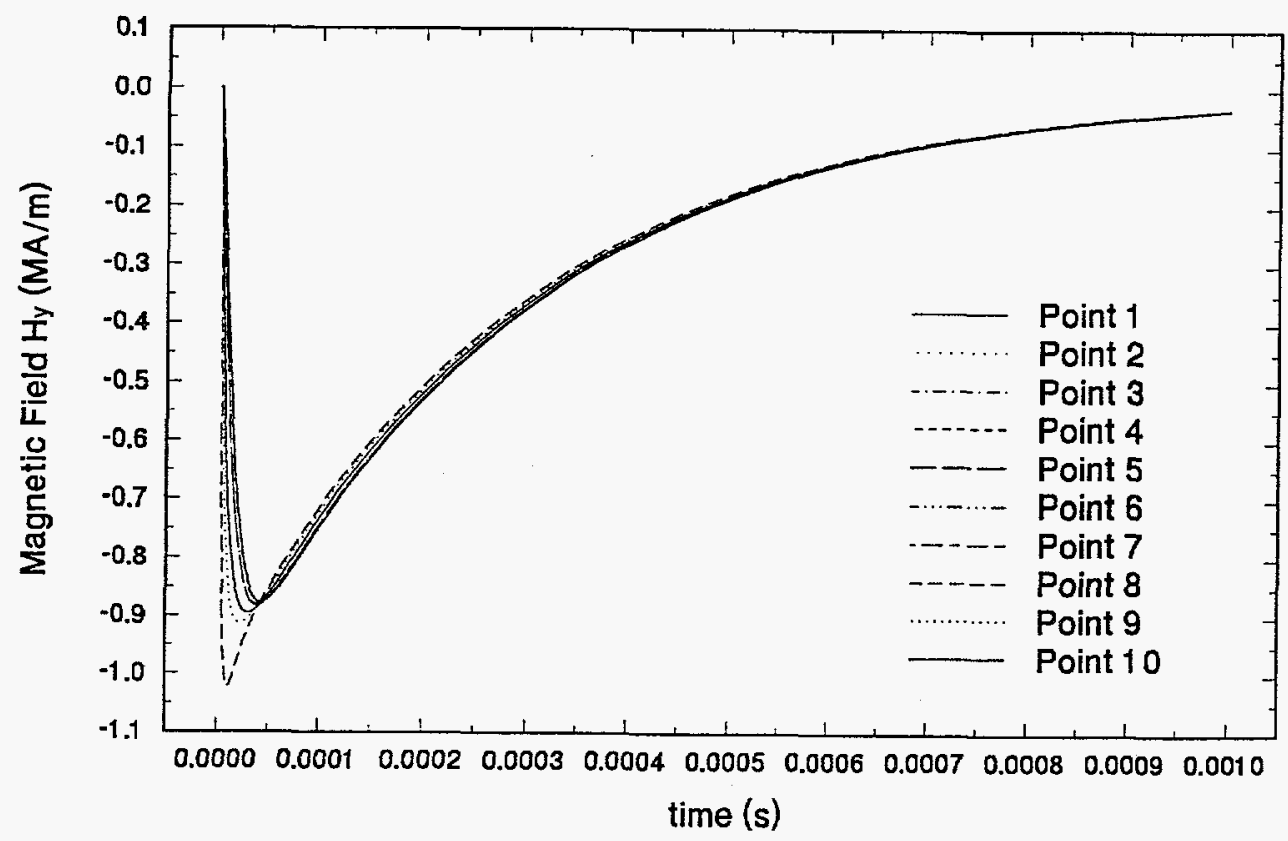

Figure 15. Finite element results for interior magnetic field (exterior uniform field is $1 \mathrm{MA} / \mathrm{m}$ ) with stainless steel pill box barrier and air core. Graphs 3 and 4 of 5 . 


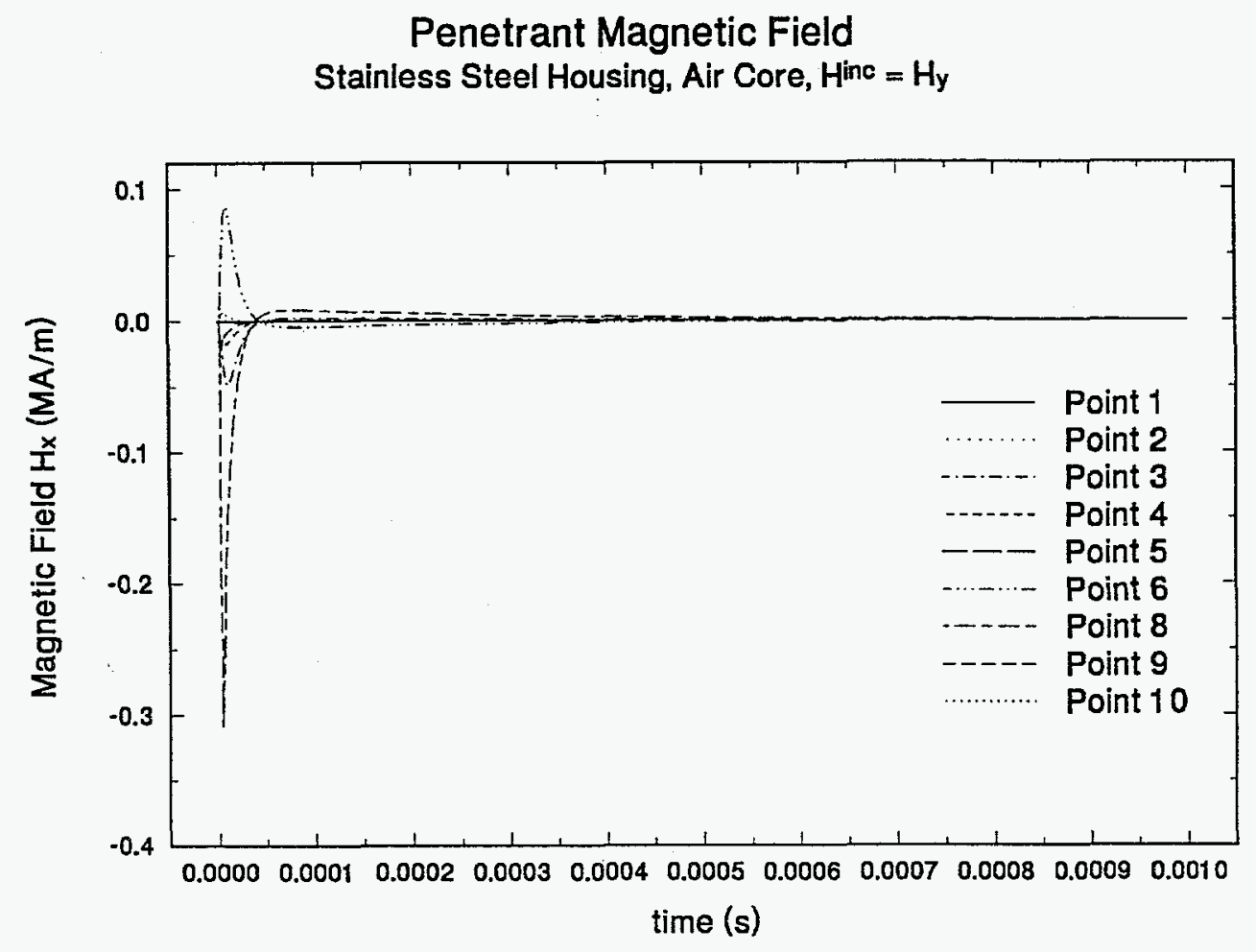

Figure 15. Finite element results for interior magnetic field (exterior uniform field is $1 \mathrm{MA} / \mathrm{m}$ ) with stainless steel pill box barrier and air core. Graph 5 of 5 . 
Penetrant Magnetic Field

Stainless Steel Housing, PMC Core, $\mathrm{Hinc}^{\mathrm{H}}=\mathrm{Hx}$

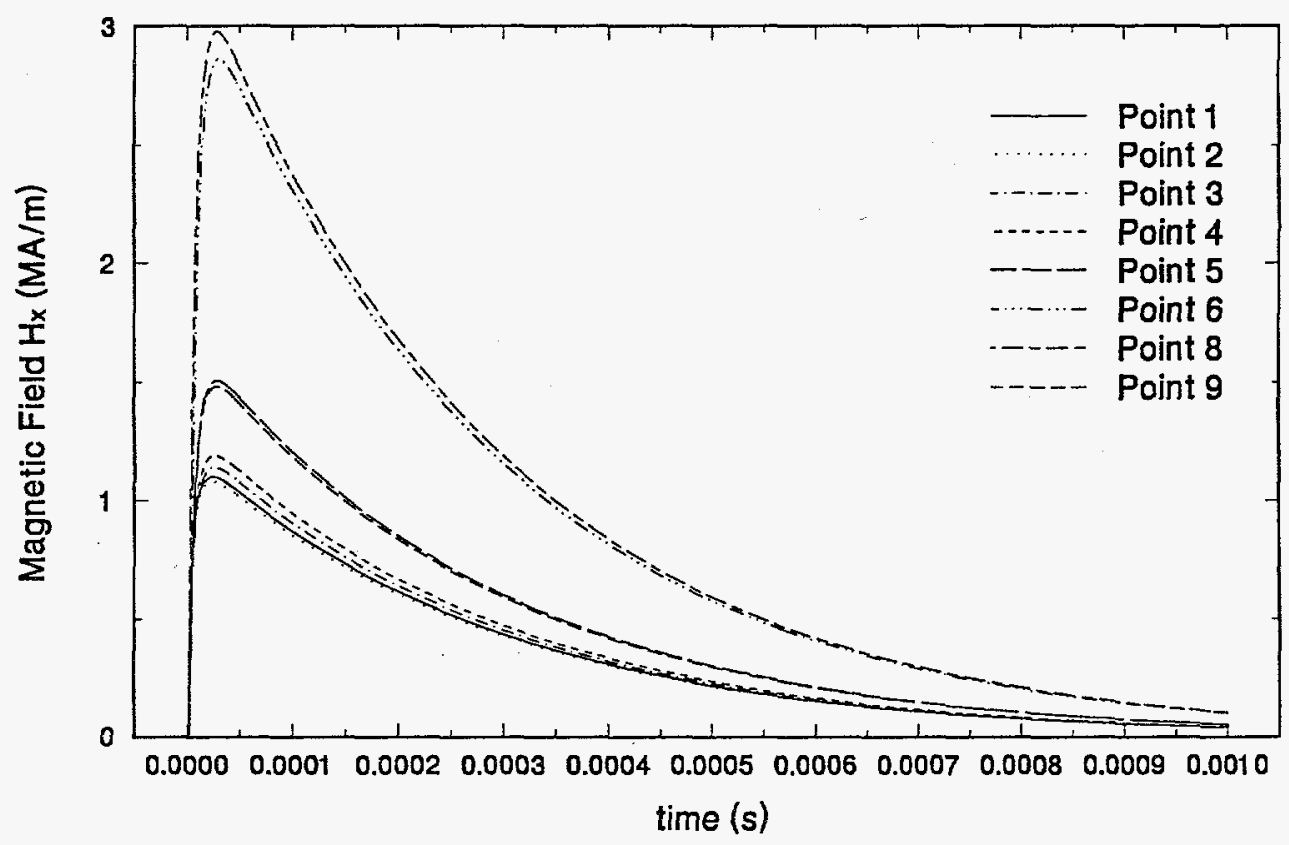

Penetrant Magnetic Field

Stainless Steel Housing, PMC Core, $\mathrm{H}^{\text {inc }}=\mathrm{Hx}$

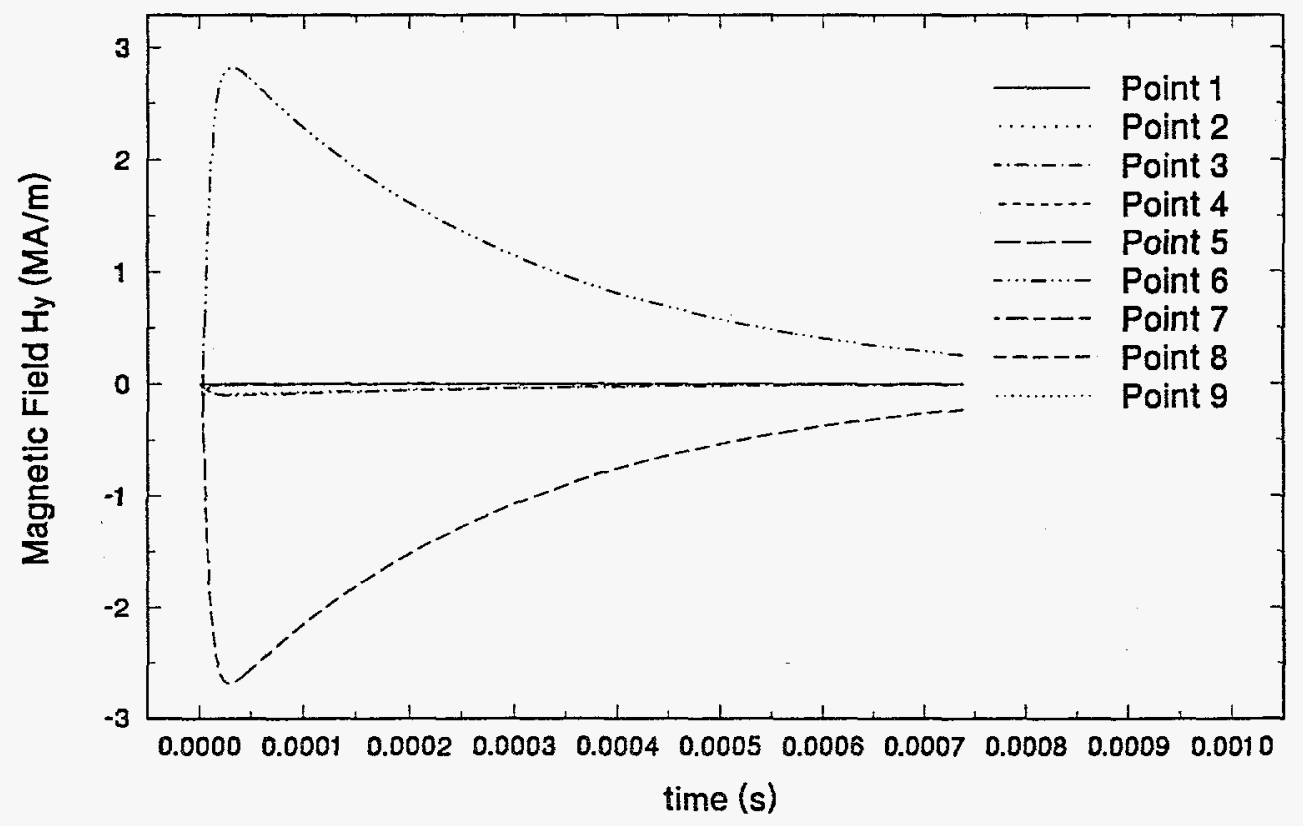

Figure 16. Finite element results for interior magnetic field (exterior uniform field is $1 \mathrm{MA} / \mathrm{m}$ ) with stainless steel pill box barrier and PMC core. Graphs 1 and 2 of 4 . 


\section{Penetrant Magnetic Field}

Stainless Steel Housing, PMC Core, $\mathrm{Hine}=\mathrm{Hy}$

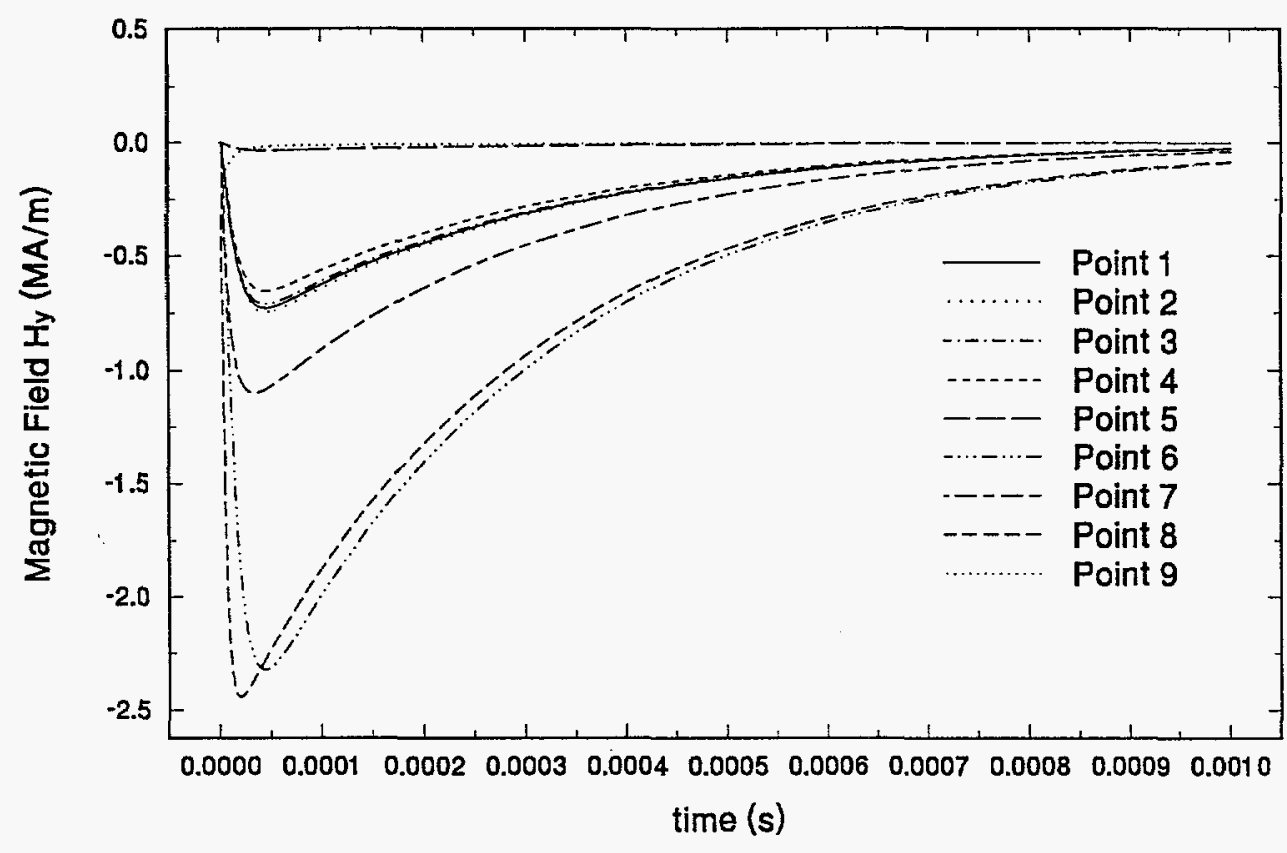

Penetrant Magnetic Field Stainless Steel Housing, PMC Core, $\mathrm{H}^{\text {inc }}=\mathrm{Hy}$

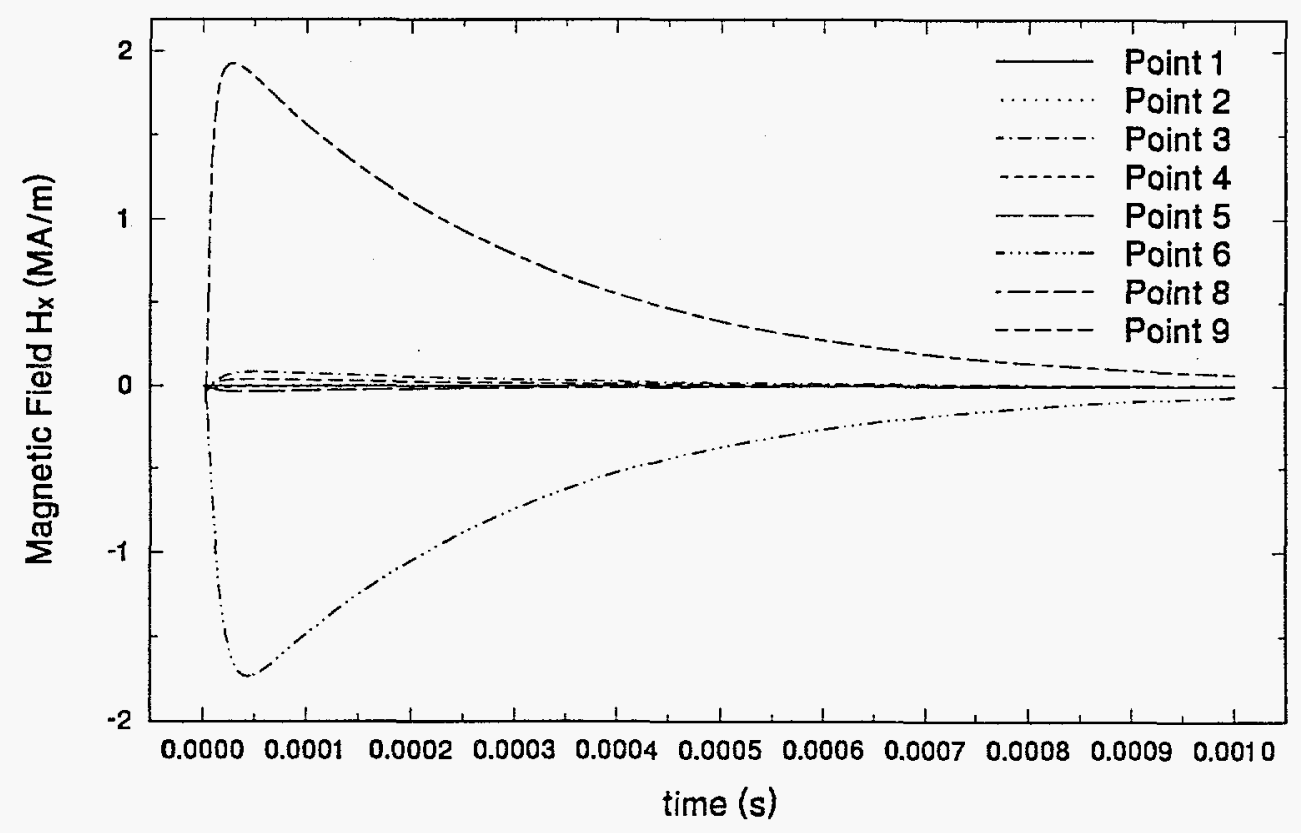

Figure 16. Finite element results for interior magnetic field (exterior uniform field is $1 \mathrm{MA} / \mathrm{m}$ ) with stainless steel pill box barrier and PMC core. Graphs 3 and 4 of 4 . 


\section{Penetrant Magnetic Field Derivative \\ Aluminum Housing, Air Core, $\mathrm{H}^{\text {inc }}=\mathrm{Hx}$}

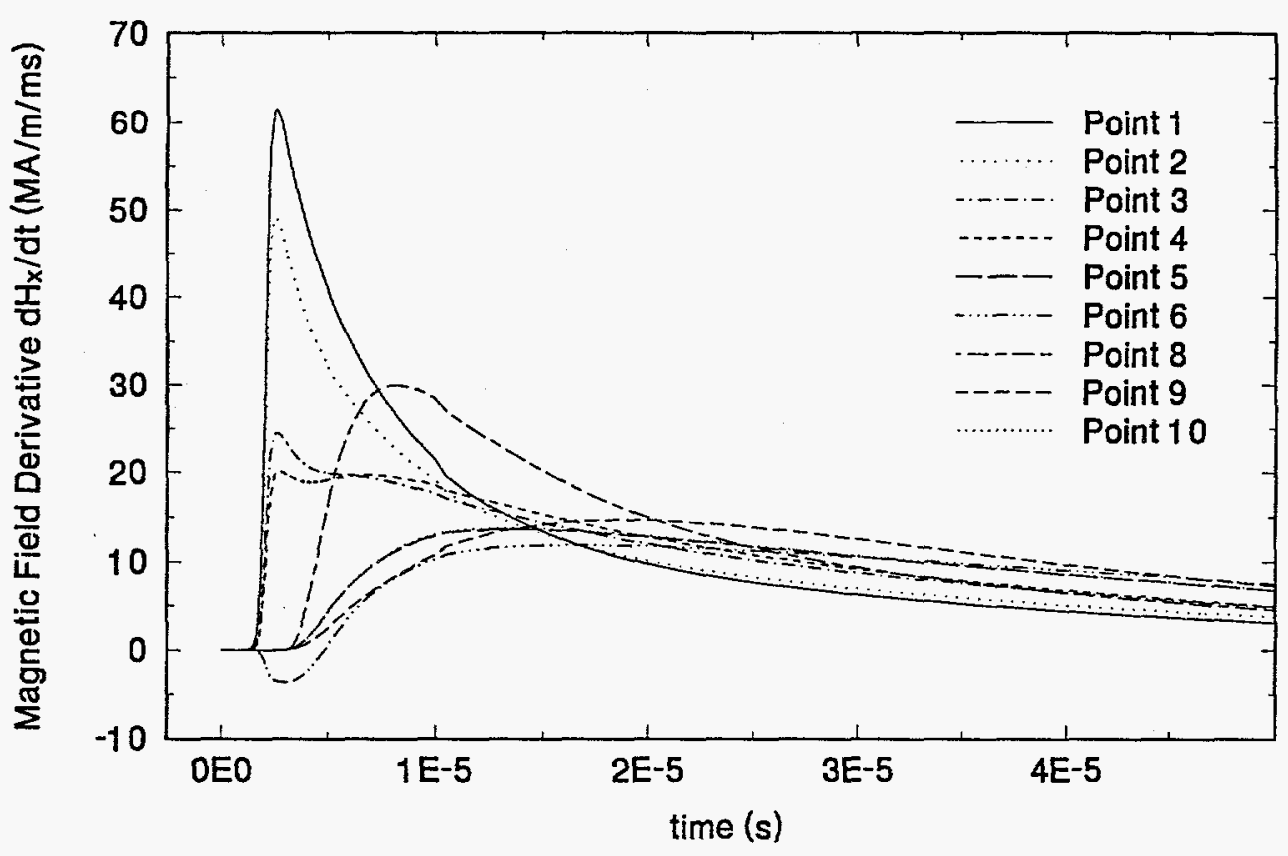

Penetrant Magnetic Field Derivative

Aluminum Housing, Air Core, $\mathrm{Hinc}^{\text {inc }} \mathrm{Hx}$

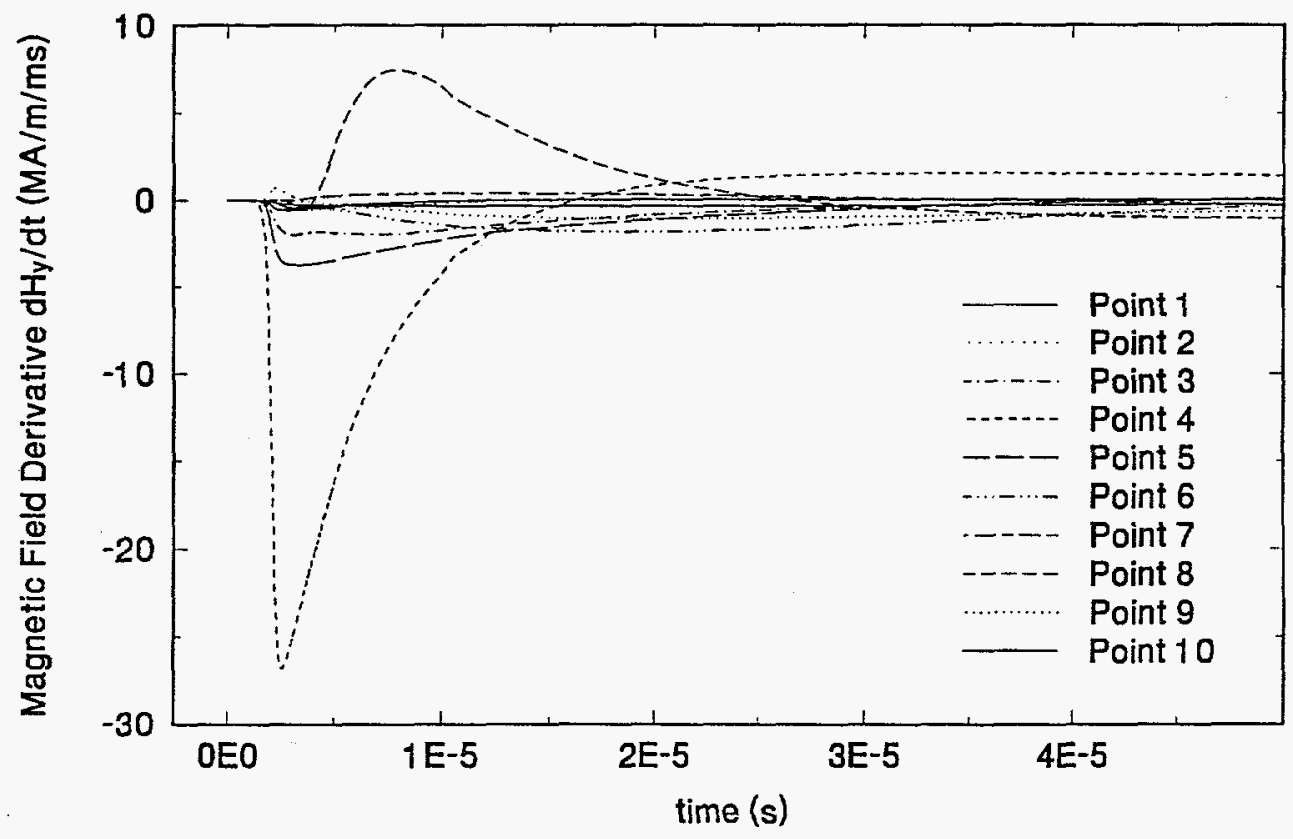

Figure 17. Finite element results for interior magnetic field time derivative (exterior uniform field is $1 \mathrm{MA} / \mathrm{m}$ ) with aluminum pill box barrier and air core. Graphs 1 and 2 of 4 . 


\section{Penetrant Magnetic Field Derivative \\ Aluminum Housing, Air Core, $\mathrm{Hinc}=\mathrm{Hy}_{\mathrm{y}}$}

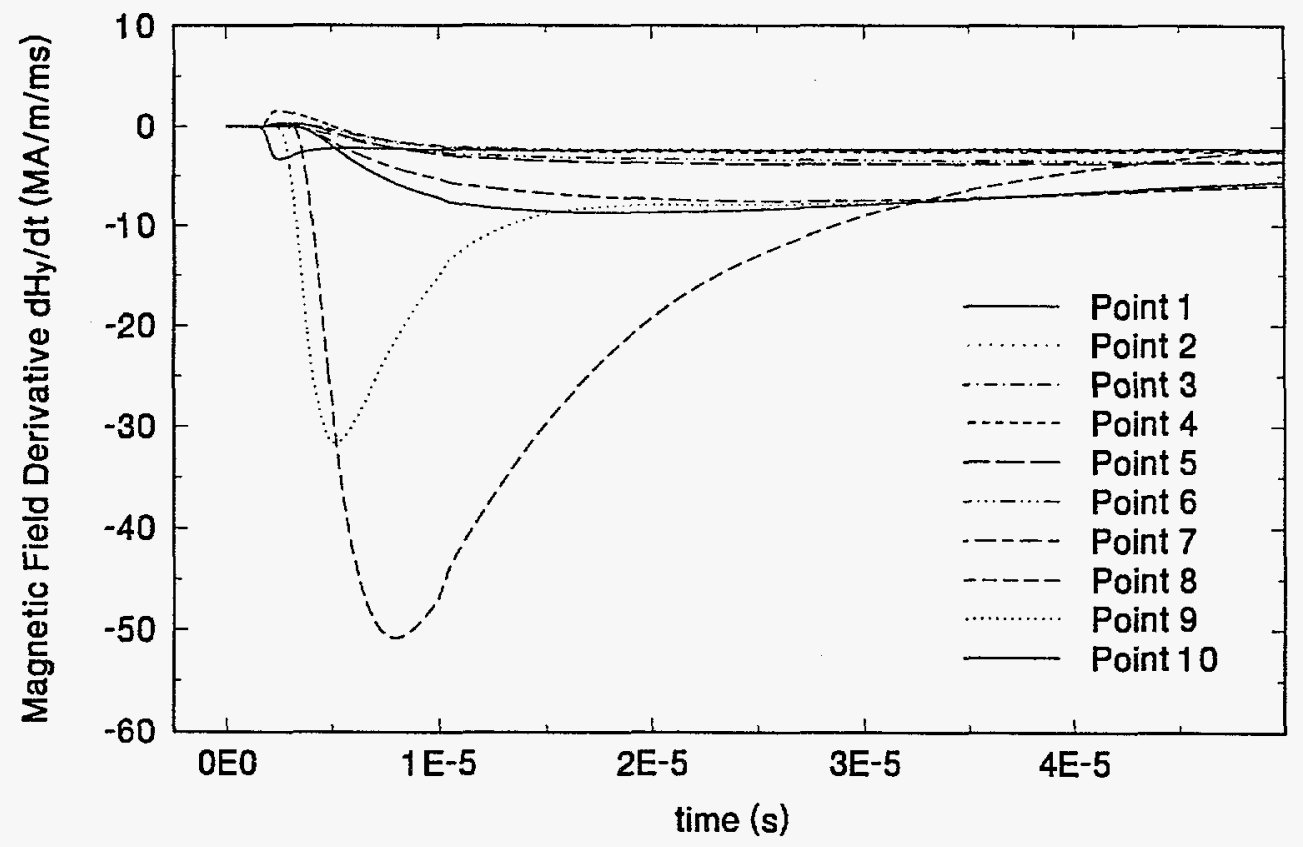

\section{Penetrant Magnetic Field Derivative \\ Aluminum Housing, Air Core, $\mathrm{Hinc}=\mathrm{Hy}$}

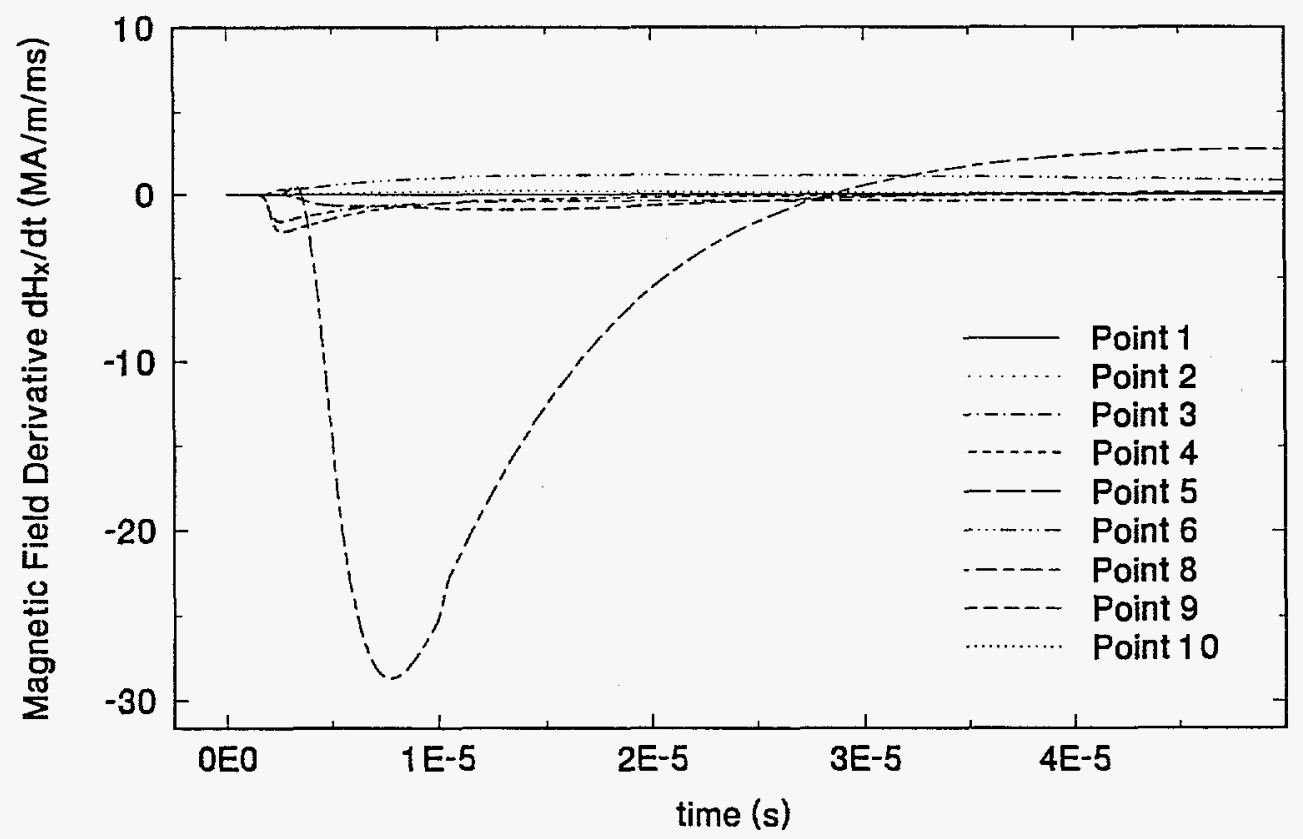

Figure 17. Finite element results for interior magnetic field time derivative (exterior uniform field is $1 \mathrm{MA} / \mathrm{m}$ ) with aluminum pill box barrier and air core. Graphs 3 and 4 of 4 . 
Penetrant Magnetic Field Derivative

Aluminum Housing, PMC Core, $\mathrm{H}^{\text {Inc }}=\mathrm{Hx}$

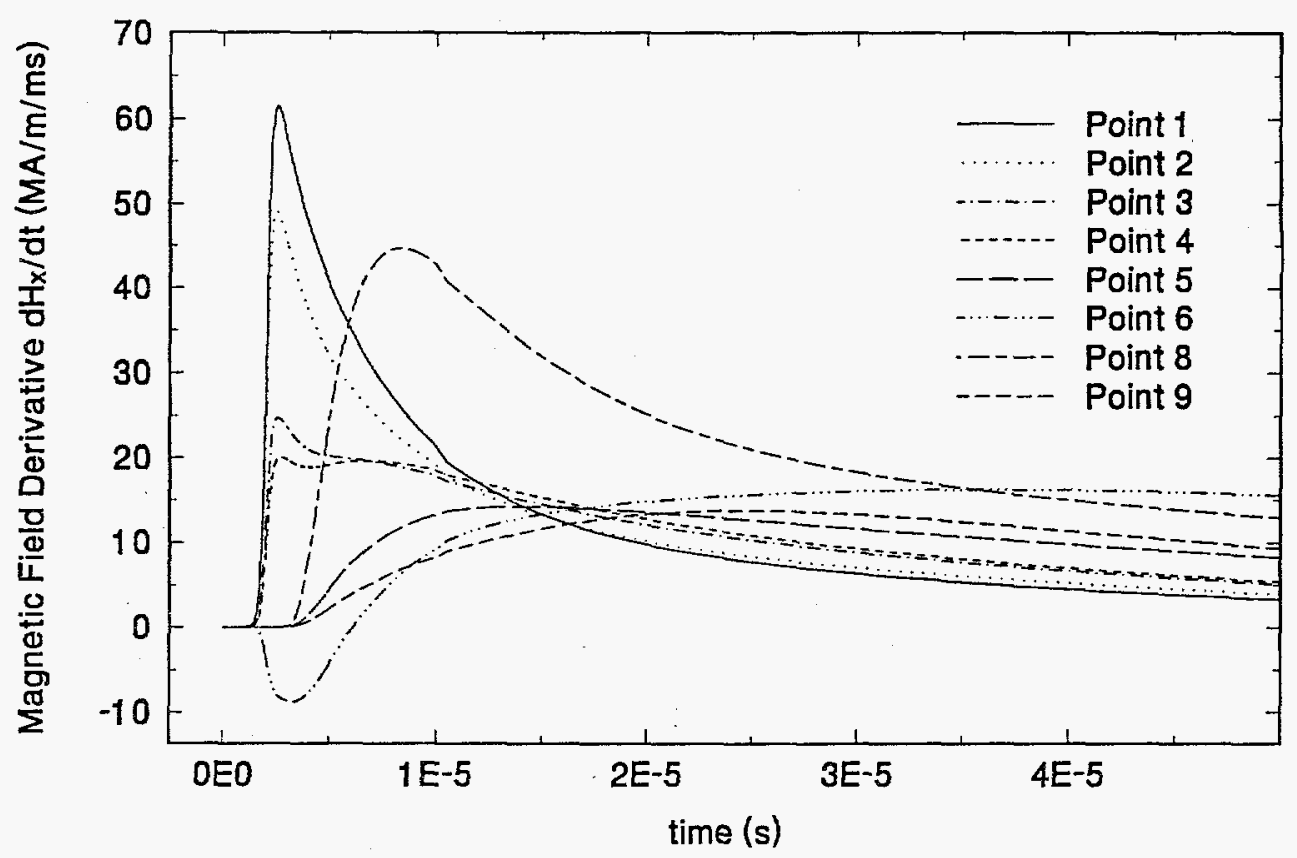

Penetrant Magnetic Field Derivative

Aluminum Housing, PMC Core, $\mathrm{H}^{\mathrm{inc}}=\mathrm{H}_{\mathrm{x}}$

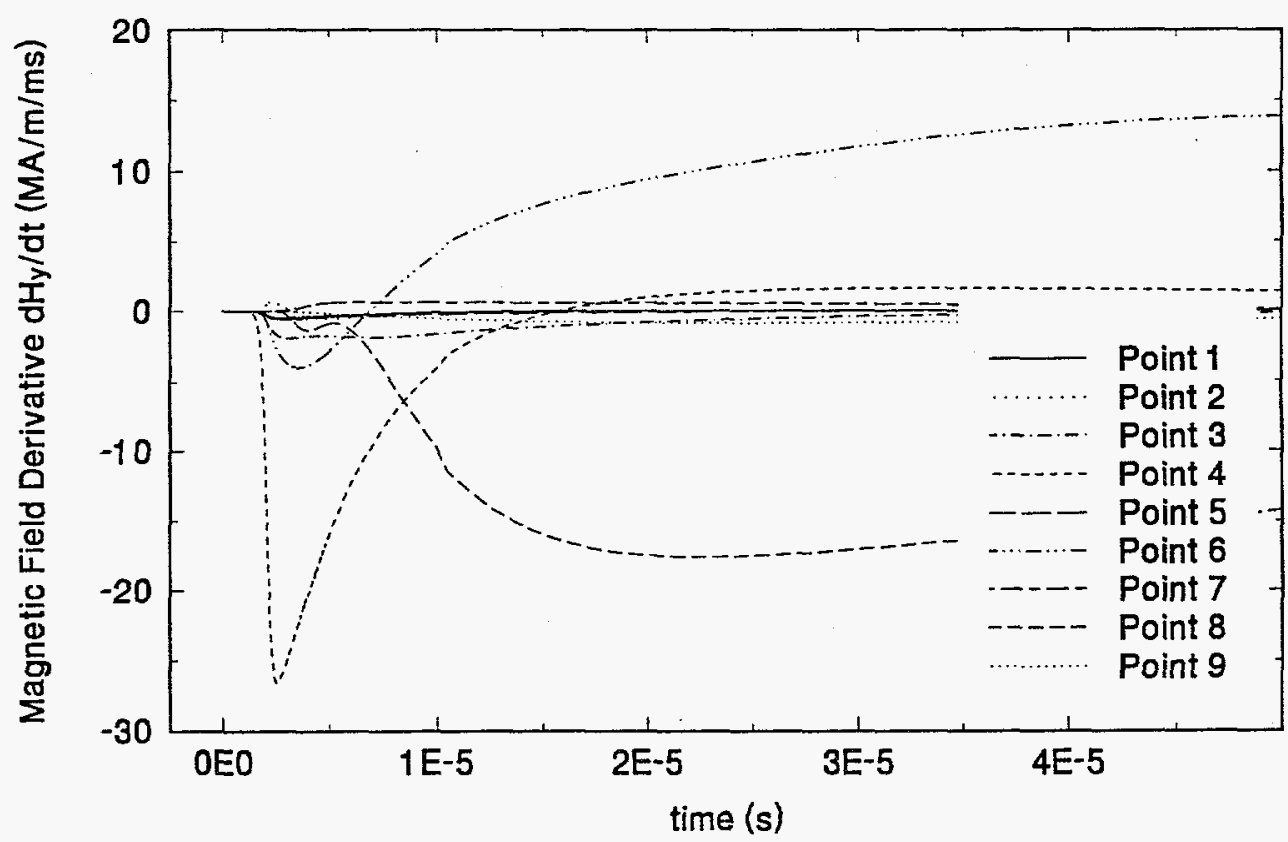

Figure 18. Finite element results for interior magnetic field time derivative (exterior uniform field is $1 \mathrm{MA} / \mathrm{m}$ ) with aluminum pill box barrier and PMC core. Graphs 1 and 2 of 4 . 


\section{Penetrant Magnetic Field Derivative \\ Aluminum Housing, PMC Core, $\mathrm{H}^{\text {live }}=\mathrm{Hy}_{\mathrm{y}}$}

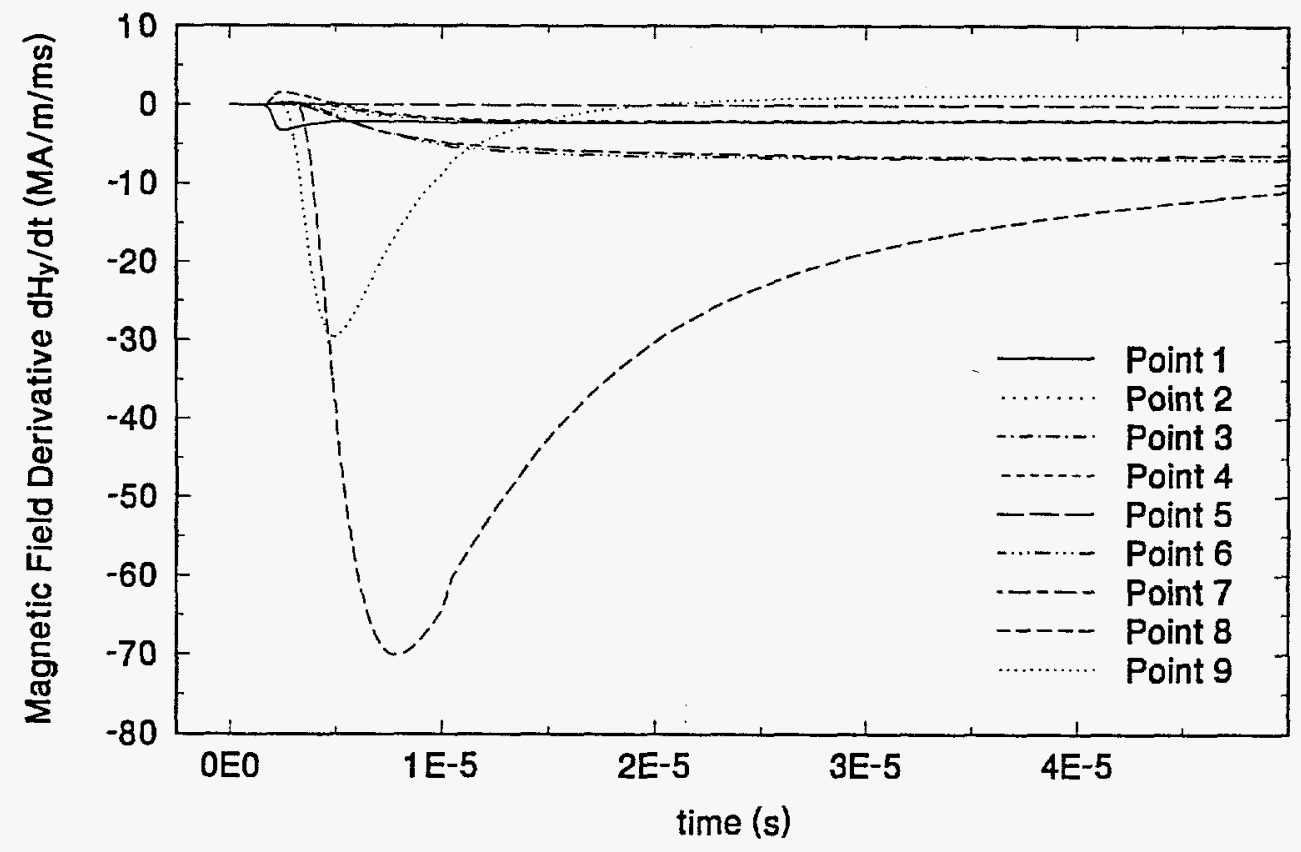

\section{Penetrant Magnetic Field Derivative Aluminum Housing, PMC Core, $\mathrm{Hinc}^{\mathrm{H}} \mathrm{Hy}$}

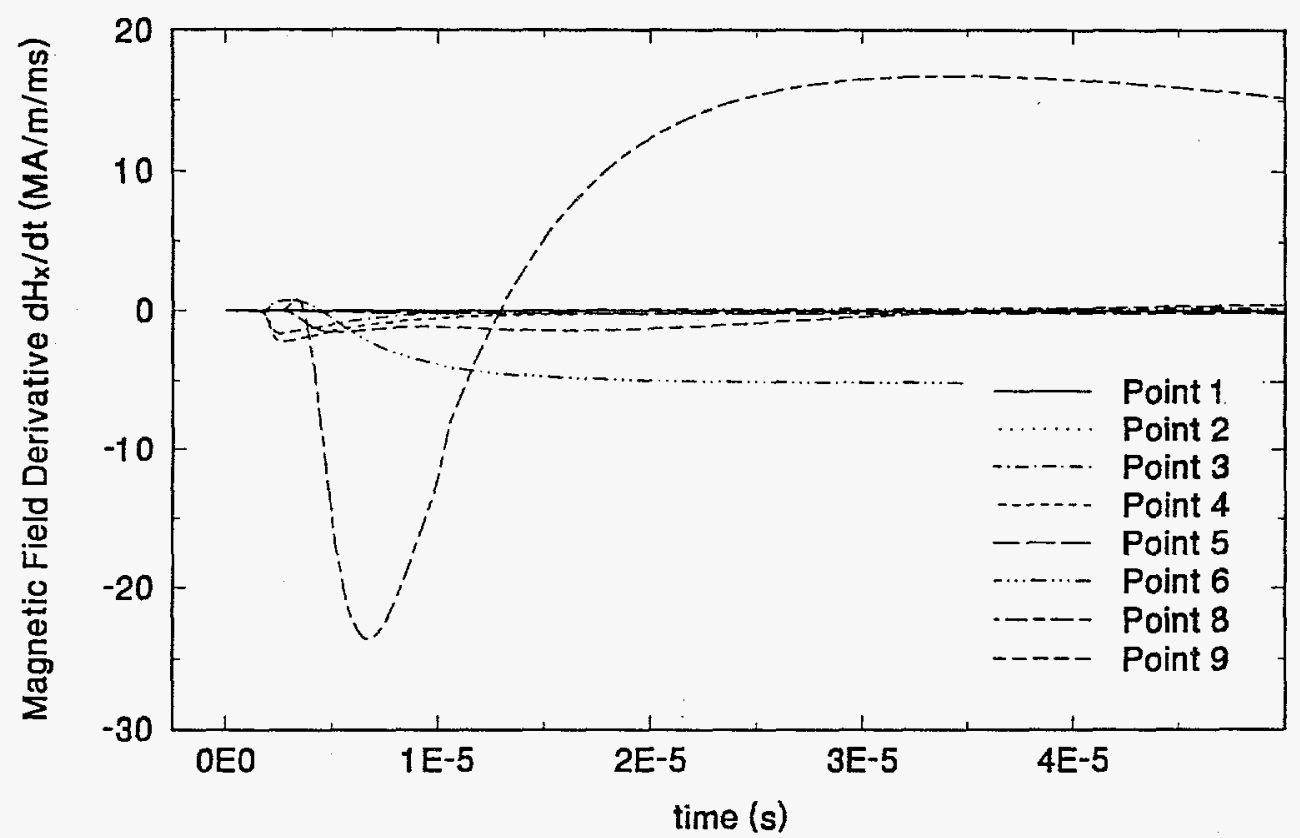

Figure 18. Finite element results for interior magnetic field time derivative (exterior uniform field is $1 \mathrm{MA} / \mathrm{m}$ ) with aluminum pill box barrier and PMC core. Graphs 3 and 4 of 4 . 
Penetrant Magnetic Field Derivative

Stainless Steel Housing, Air Core, $\mathrm{H}^{\text {inc }}=\mathrm{Hx}_{\mathrm{x}}$

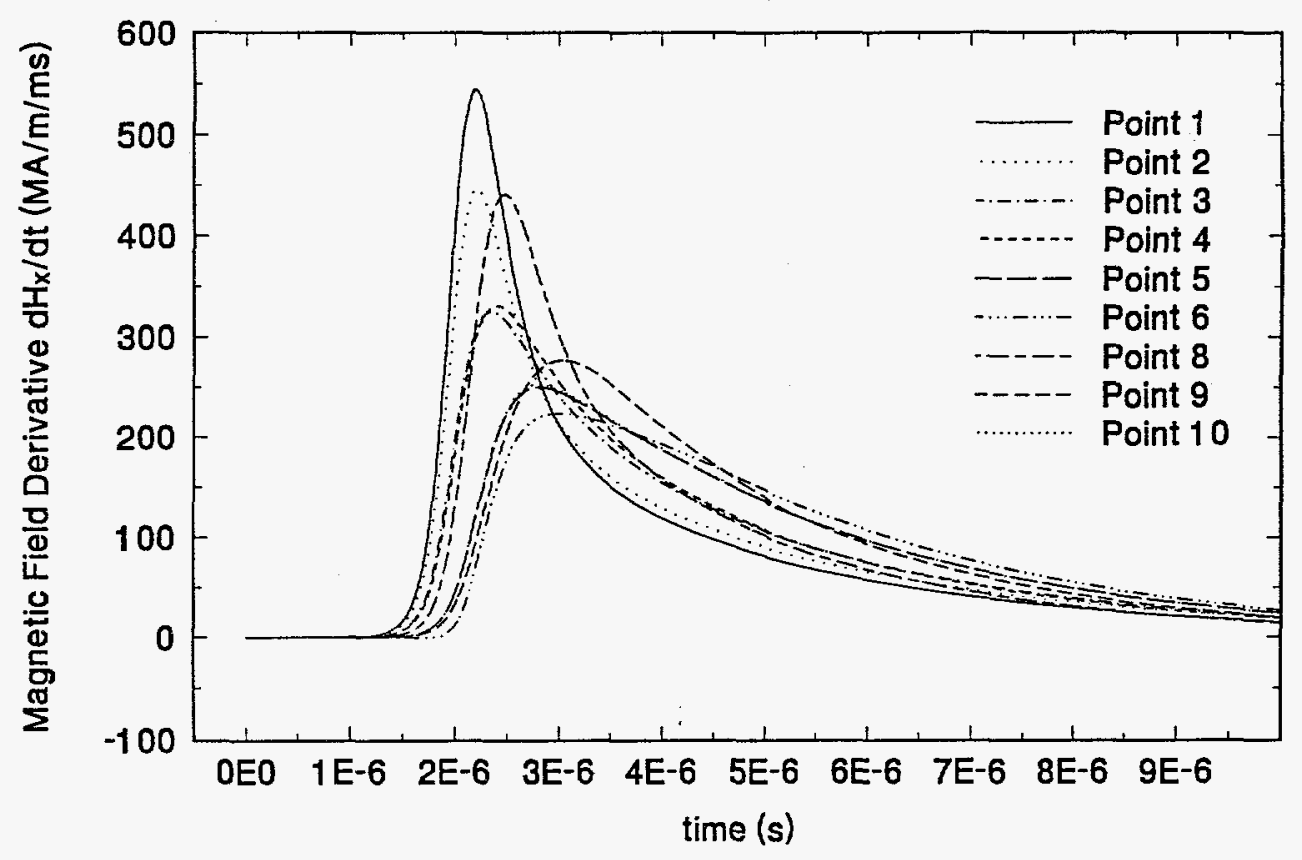

Penetrant Magnetic Field Derivative

Stainless Steel Housing, Air Core, Hinc $=\mathrm{Hx}_{\mathrm{x}}$

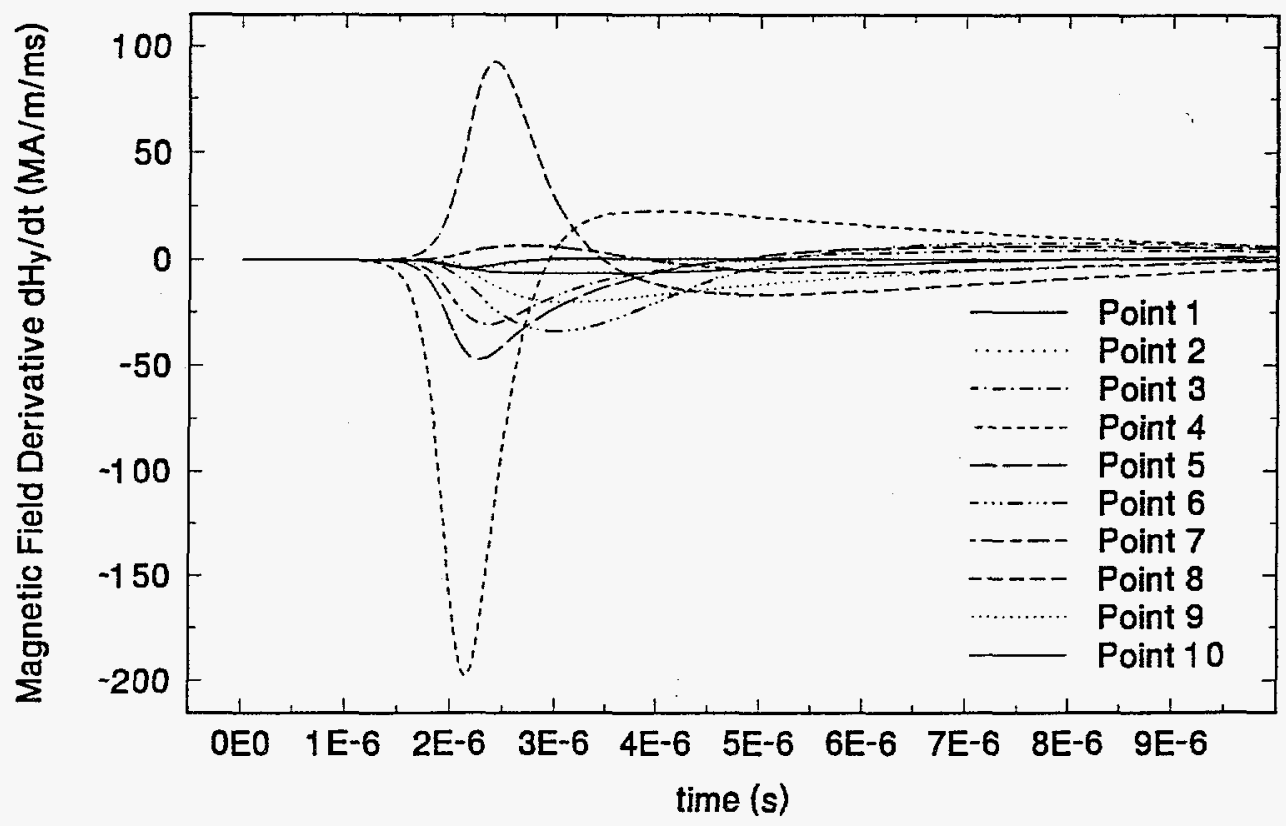

Figure 19. Finite element results for interior magnetic field time derivative (exterior uniform field is $1 \mathrm{MA} / \mathrm{m}$ ) with stainless steel pill box barrier and air core. Graphs 1 and 2 of 4 . 


\section{Penetrant Magnetic Field Derivative}

Stainless Steel Housing, Air Core, $\mathrm{H}^{\text {inc }}=\mathrm{Hy}$

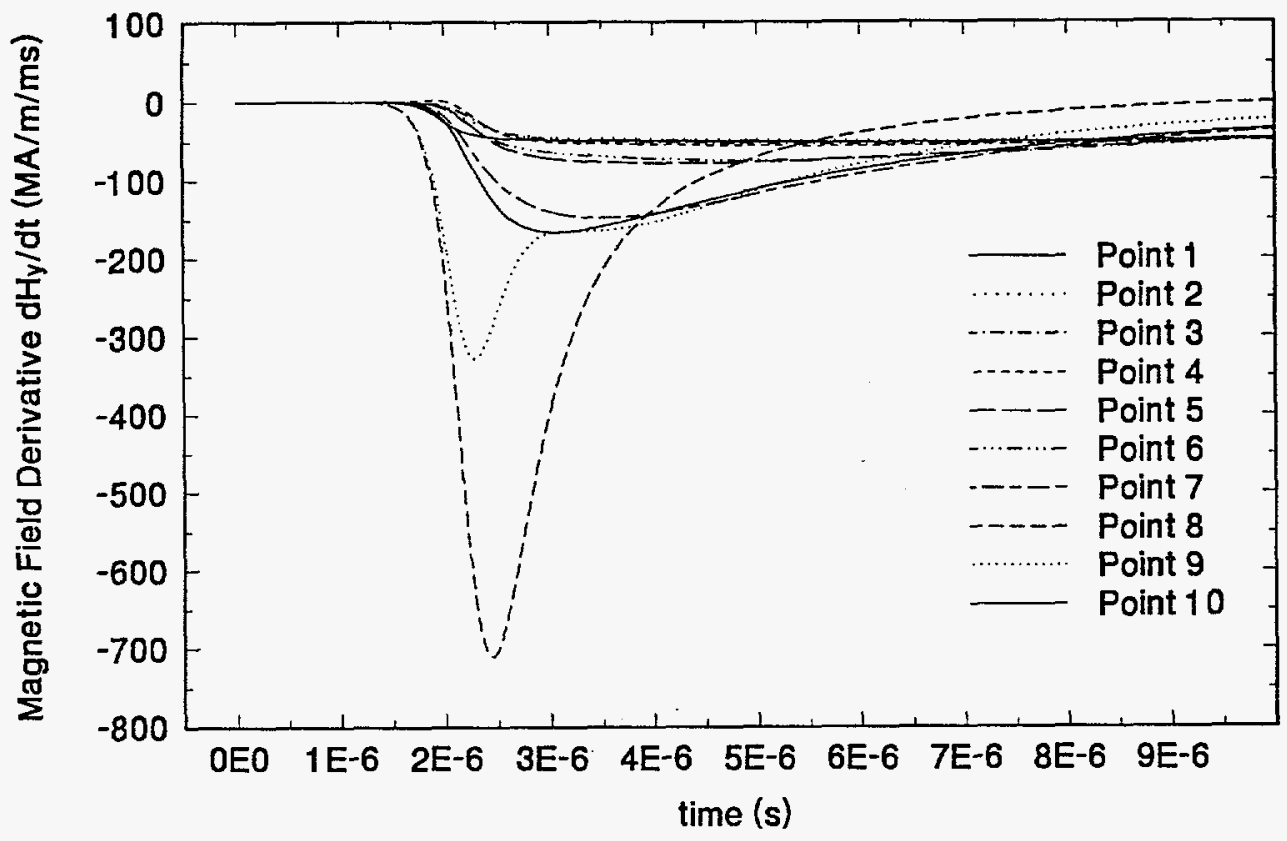

Penetrant Magnetic Field Derivative

Stainless Steel Housing, Air Core, $\mathrm{Hinc}^{\mathrm{in}} \mathrm{Hy}$

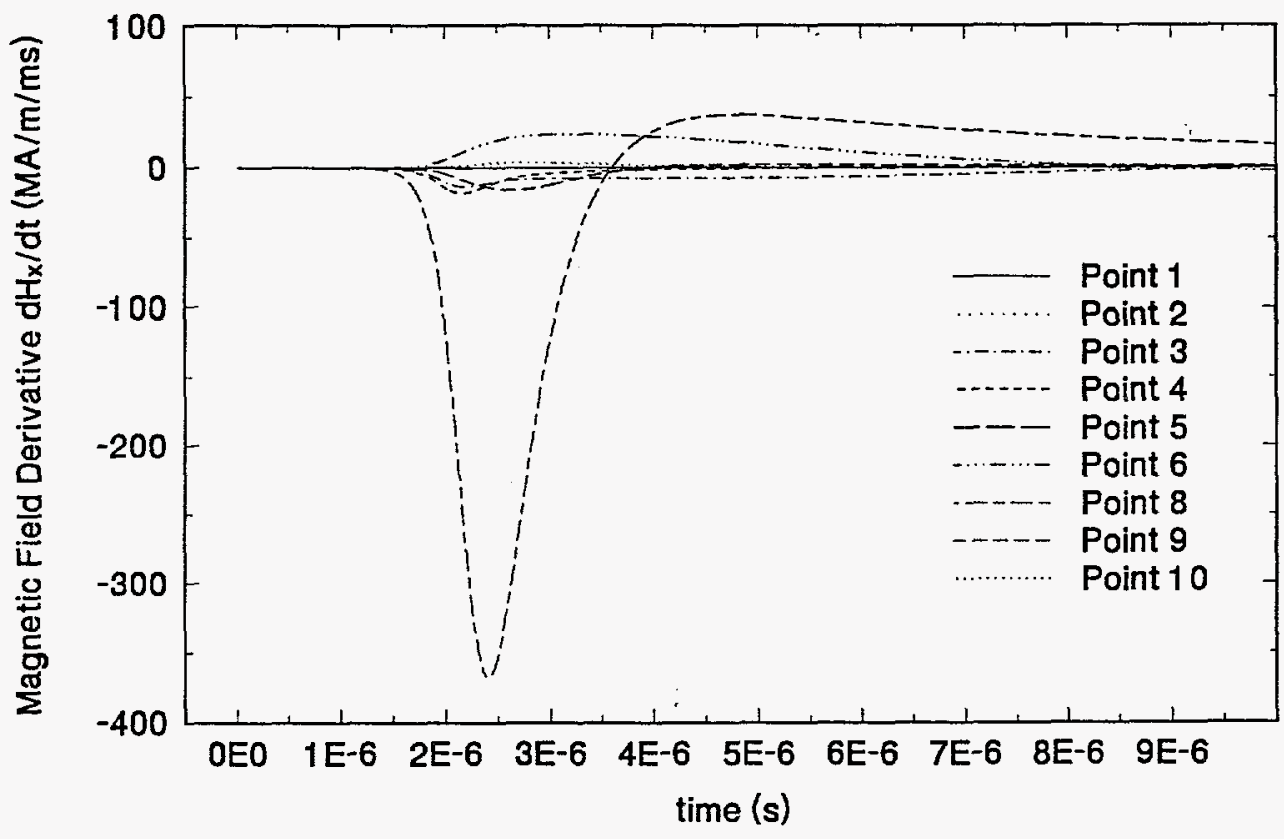

Figure 19. Finite element results for interior magnetic field time derivative (exterior uniform field is $1 \mathrm{MA} / \mathrm{m}$ ) with stainless steel pill box barrier and air core. Graphs 3 and 4 of 4 . 
Penetrant Magnetic Field Derivative

Stainless Steel Housing, PMC Core, $\mathrm{H}^{\text {inc }}=\mathrm{Hx}_{\mathbf{x}}$

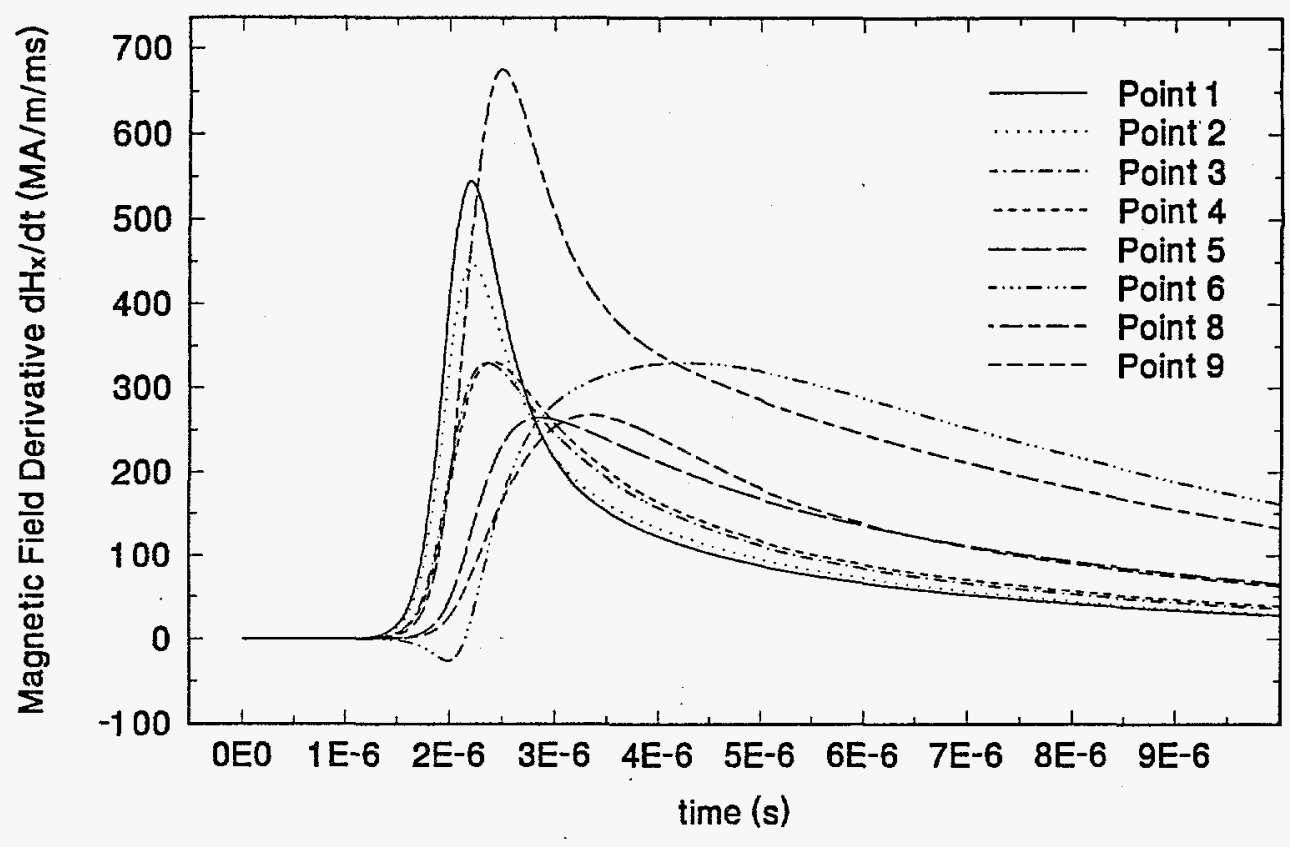

Penetrant Magnetic Field Derivative Stainless Steel Housing, PMC Core, $\mathrm{H}^{\mathrm{lnc}}=\mathrm{H}_{\mathrm{x}}$

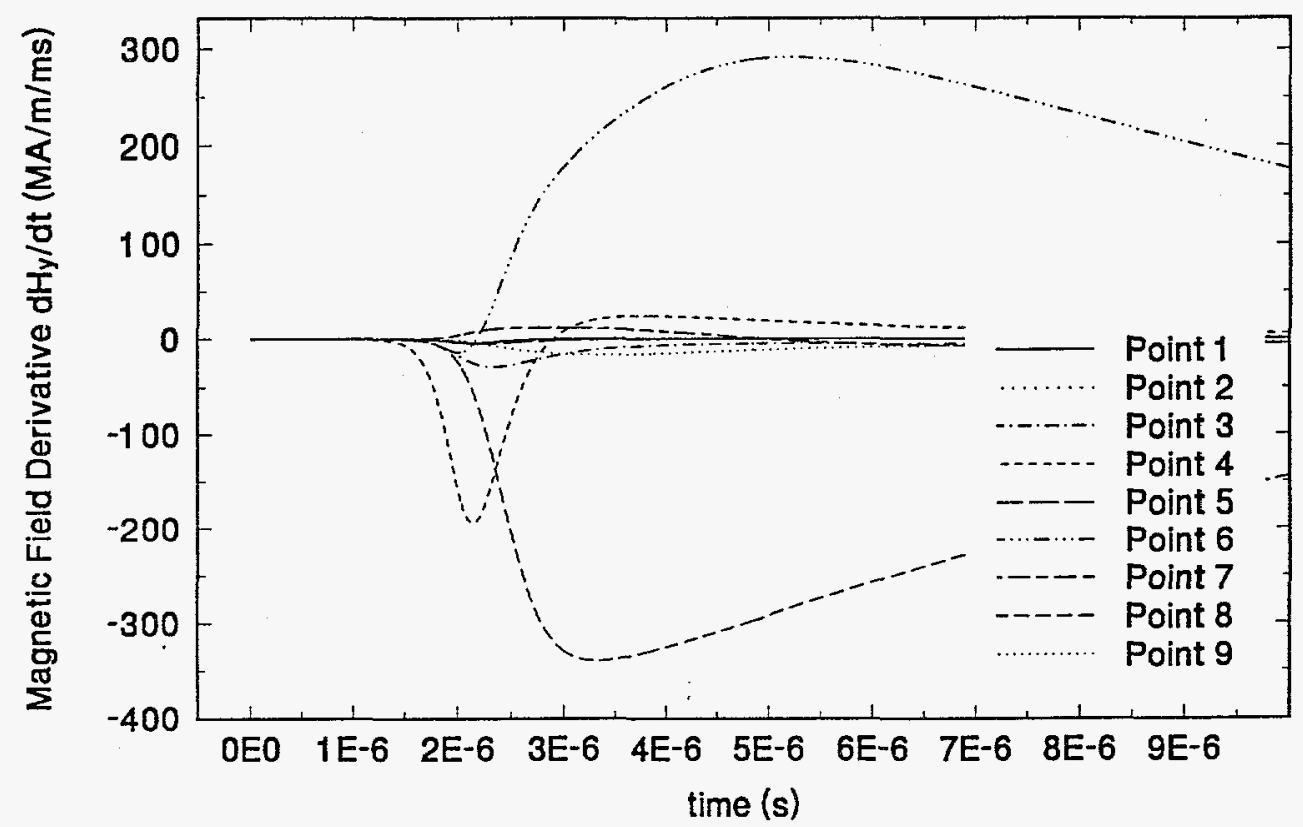

Figure 20. Finite element results for interior magnetic field time derivative (exterior uniform field is $1 \mathrm{MA} / \mathrm{m}$ ) with stainless steel pill box barrier and PMC core. Graphs 1 and 2 of 4 . 


\section{Penetrant Magnetic Field Derivative \\ Stainless Steel Housing, PMC Core, $\mathrm{H}^{\text {inc }}=\mathrm{Hy}$}

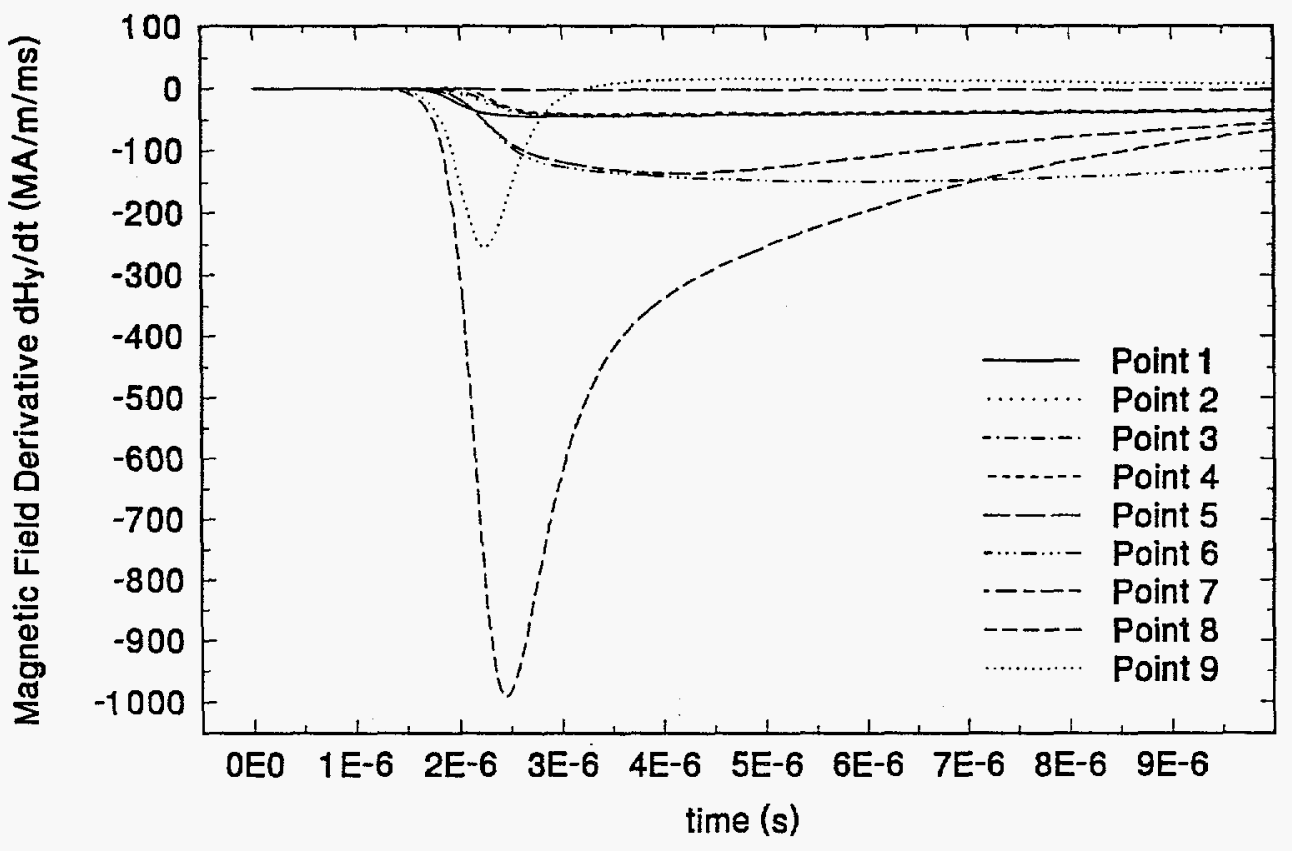

Penetrant Magnetic Field Derivative

Stainless Steel Housing, PMC Core, $H^{\text {inc }}=H_{y}$

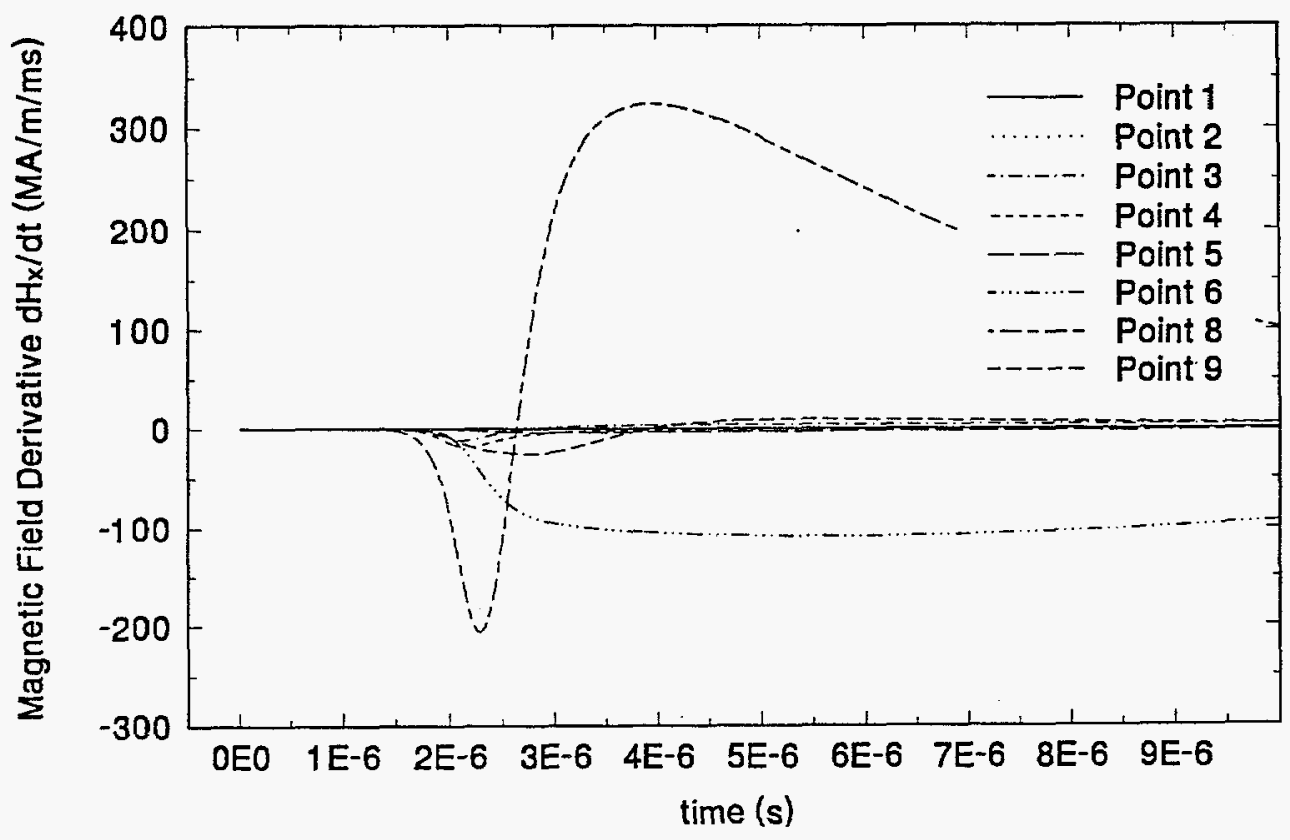

Figure 20. Finite element results for interior magnetic field time derivative (exterior uniform field is $1 \mathrm{MA} / \mathrm{m}$ ) with stainless steel pill box barrier and PMC core. Graphs 3 and 4 of 4 . 
hole would be zero if the remainder of the shield were perfectly conducting) point 4 provides an upper estimate on the foil penetration with total field derivative $3.4 \times 10^{10} \mathrm{~A} / \mathrm{m} / \mathrm{sec}$, which is slightly greater than twice the nominal interior level. Scaling this up to the previous field level (2.2 $\mathrm{MA} / \mathrm{m}$ ) the result is slightly less than that anticipated in the previous section (36) (at twice the foil radius) on the analytical estimates for foil penetration of the aluminum shield. Similarly if we ignore test points $1,2,3,4$, and 8 we see from the stainless steel results that $2.5 \times 10^{11} \mathrm{~A} / \mathrm{m} / \mathrm{sec}$ is a good estimate of the remaining curves with the exception of point 9 which has a value $3.3 \times 10^{11}$ $\mathrm{A} / \mathrm{m} / \mathrm{sec}$. Extrapolating the first value to the field level (7) thus gives

$$
\frac{\partial}{\partial t} H_{\text {code }} \approx 2.5 \times 10^{11} \mathrm{~A} / \mathrm{m} / \mathrm{sec}(2.2) \approx 5.5 \times 10^{11} \mathrm{~A} / \mathrm{m} / \mathrm{sec} \text { (stainless steel) }
$$

which again is quite close to the analytical estimate (15). Extrapolating point 9 to the this incident field level gives

$$
\frac{\partial}{\partial t} H_{\text {code }} \approx 3.3 \times 10^{11} \mathrm{~A} / \mathrm{m} / \mathrm{sec}(2.2) \approx 7.3 \times 10^{11} \mathrm{~A} / \mathrm{m} / \mathrm{sec} \text { (stainless steel) }
$$

which is slightly less than fifty percent greater than the analytic estimate, but appears to be confined to the outer boundary region. The corner (point 8) total field derivative for the stainless steel housing and air core is bounded by $8 \times 10^{11} \mathrm{~A} / \mathrm{m} / \mathrm{sec}$. This is slightly more than three times the nominal interior field level $\left(2.5 \times 10^{11} \mathrm{~A} / \mathrm{m} / \mathrm{sec}\right)$; thus again the crude factor of two corner enhancement, previously estimated from Kaden's time harmonic results, somewhat underestimates the time domain corner enhancement (again this enhancement is only visible at the corner point 8). The total field derivative at point 4 is $3.8 \times 10^{11} \mathrm{~A} / \mathrm{m} / \mathrm{sec}$, which is less than twice the nominal interior field derivative value. The analytical estimate of the previous section gave a smaller than nominal value at twice the radius of the foil hole (33) (if the analytical result is extrapolated up by a factor of 4 for the closer distance, and down by a factor of 2.2 for the incident field level, the previous result is near the code result).

Figures 21 and 22 are summaries showing the total interior field and total interior field derivative for the stainless steel enclosure with air core. Several points directly above the foil are not shown since they are not particularly relevant for the circuit board or transformer locations. Figure 22 clearly shows the corner enhancement compared to the nominal analytic value (15). Figure 22a also shows the enhancement at point 4 resulting from its proximity to the foil (the analytic value is the time derivative of (32) evaluated at $\left.\rho=b_{f}\right)$.

Figures 23 and 24 show the magnetic flux per unit length and its time derivative coupling into the bottom half (from point 5 to point 9 ) and bottom end cap (from point 6 to point 8 ) of the PMC high permeability transformer. The corner enhancements are visible (they increase the rise rate of the end cap flux by approximately $10 \%-20 \%$, this is an overestimate because of the two dimensional geometry and furthermore will not couple into the central core of the transformer). If we multiply the time derivative of the bottom end cap flux per unit length by the dimension $2 b_{t} \approx 7 \mathrm{~mm}$ we find (extrapolating to the field level (7))

$$
\begin{gathered}
\frac{\partial}{\partial t} \Phi \approx(7 \mathrm{~mm})(2.2) 104 \mathrm{~Wb} / \mathrm{m} / \mathrm{sec} \approx 1.6 \mathrm{~Wb} / \mathrm{sec}(\text { commercial aluminum) } \\
\frac{\partial}{\partial t} \Phi \approx(7 \mathrm{~mm})(2.2) 1880 \mathrm{~Wb} / \mathrm{m} / \mathrm{sec} \approx 29 \mathrm{~Wb} / \mathrm{sec} \text { (stainless steel) }
\end{gathered}
$$

The analytical estimates for this quantity from the previous transformer section, using $\mu_{0} \pi b_{t}^{2}$ times (59) times (15) are $2.9 \mathrm{~Wb} / \mathrm{sec}$ (commercial aluminum) and $51 \mathrm{~Wb} / \mathrm{sec}$ (stainless steel). Thus the analytical estimates are larger than the code results by less than a factor of two. 
Penetrant Magnetic Field

Stainless Steel Enclosure, Air Core, $\mathrm{H}^{\mathrm{lnc}}=\mathrm{H}_{\mathrm{x}}=2.2 \mathrm{MA} / \mathrm{m}$

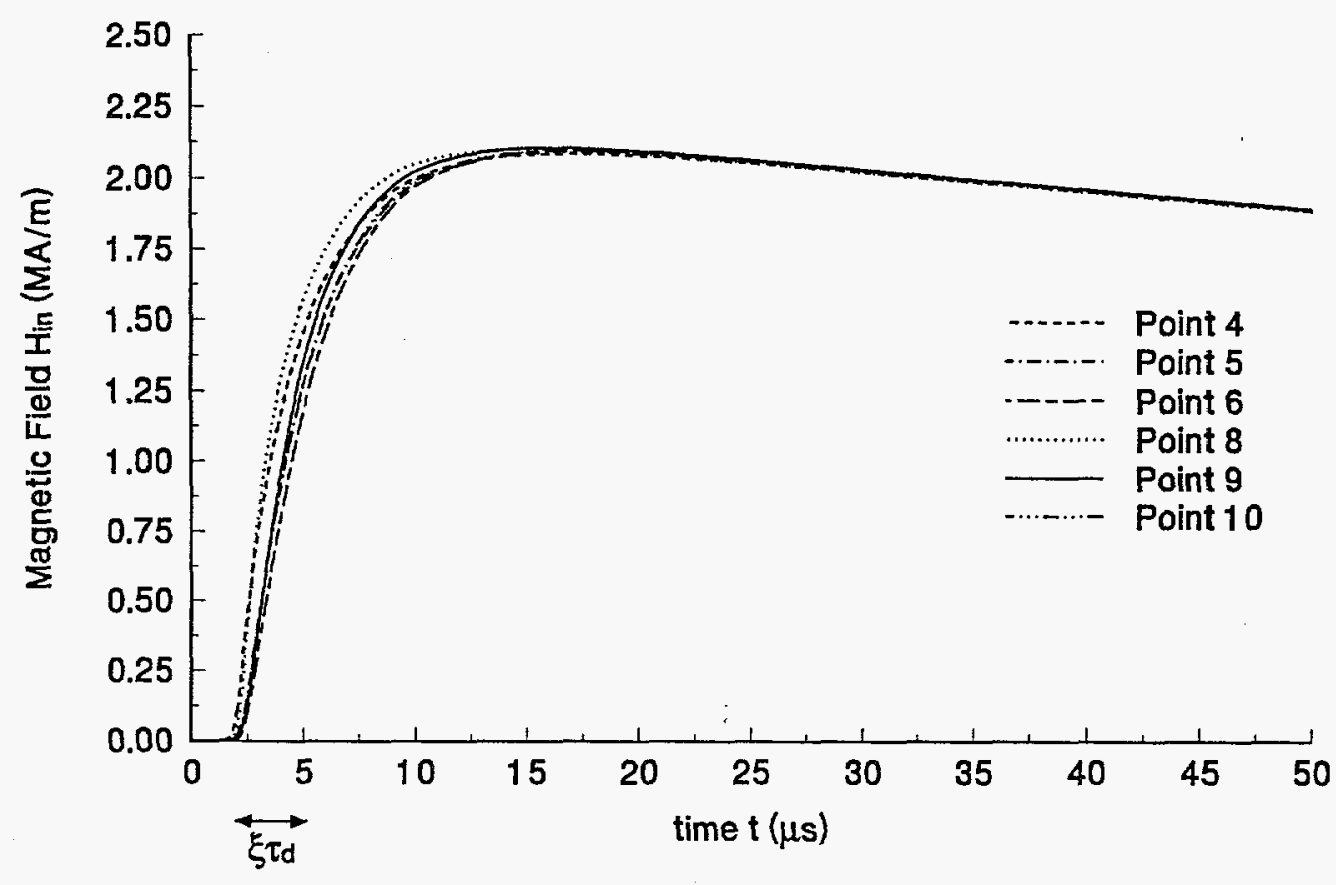

Penetrant Magnetic Field

Stainless Steel Enclosure, Air Core, $\mathrm{Hinc}_{\mathrm{in}}=\mathrm{H}=2.2 \mathrm{MA} / \mathrm{m}$

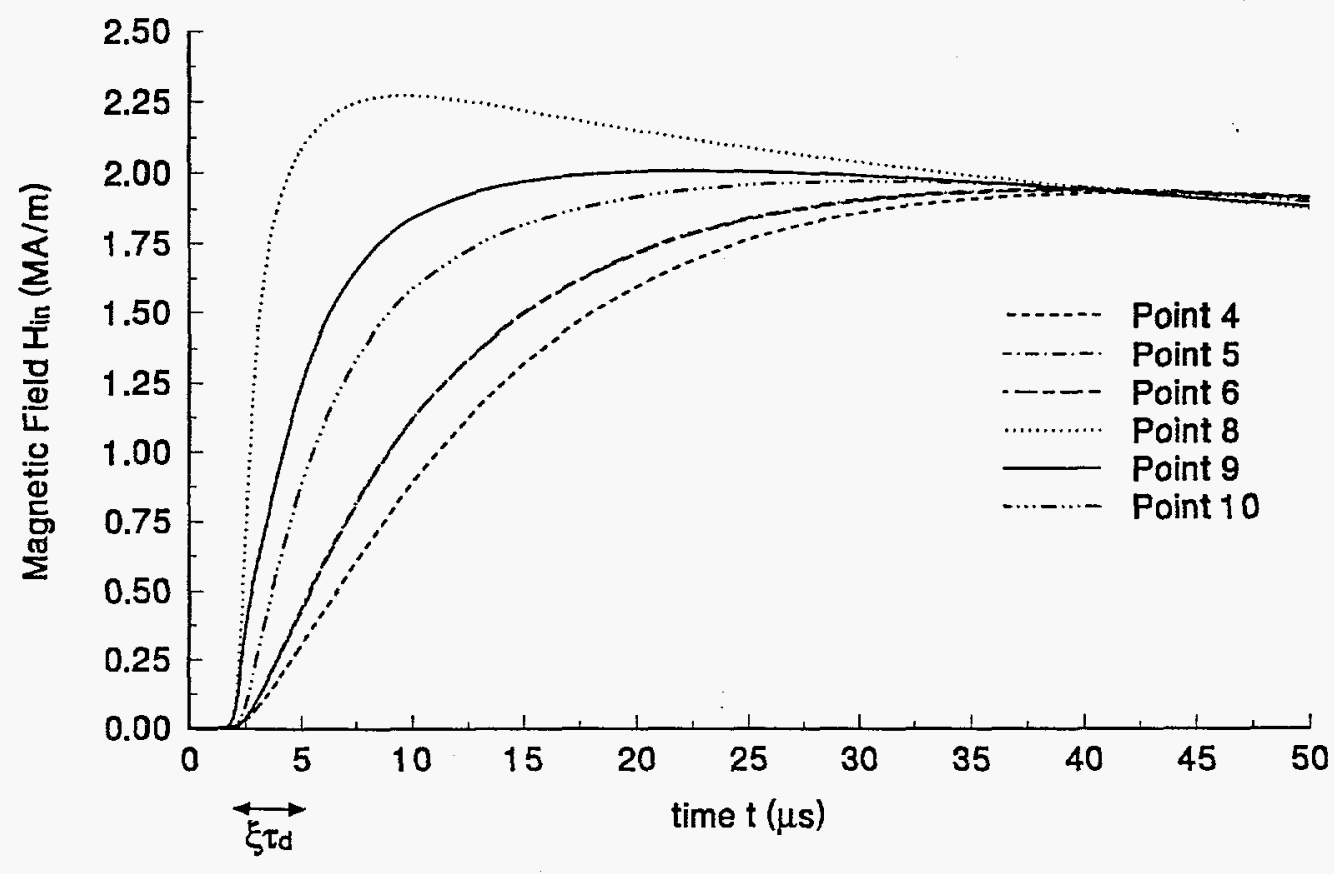

Figure 21. Summary of stainless steel air core finite element results for total penetrant field. a) Incident $x$ polarization, $\mathrm{b}$ ) Incident $y$ polarization. 
Penetrant Magnetic Field Derivative

Stainless Steel Enclosure, Air Core, $\mathrm{H}_{\text {inc }}=\mathrm{H} x=2.2 \mathrm{MA} / \mathrm{m}$

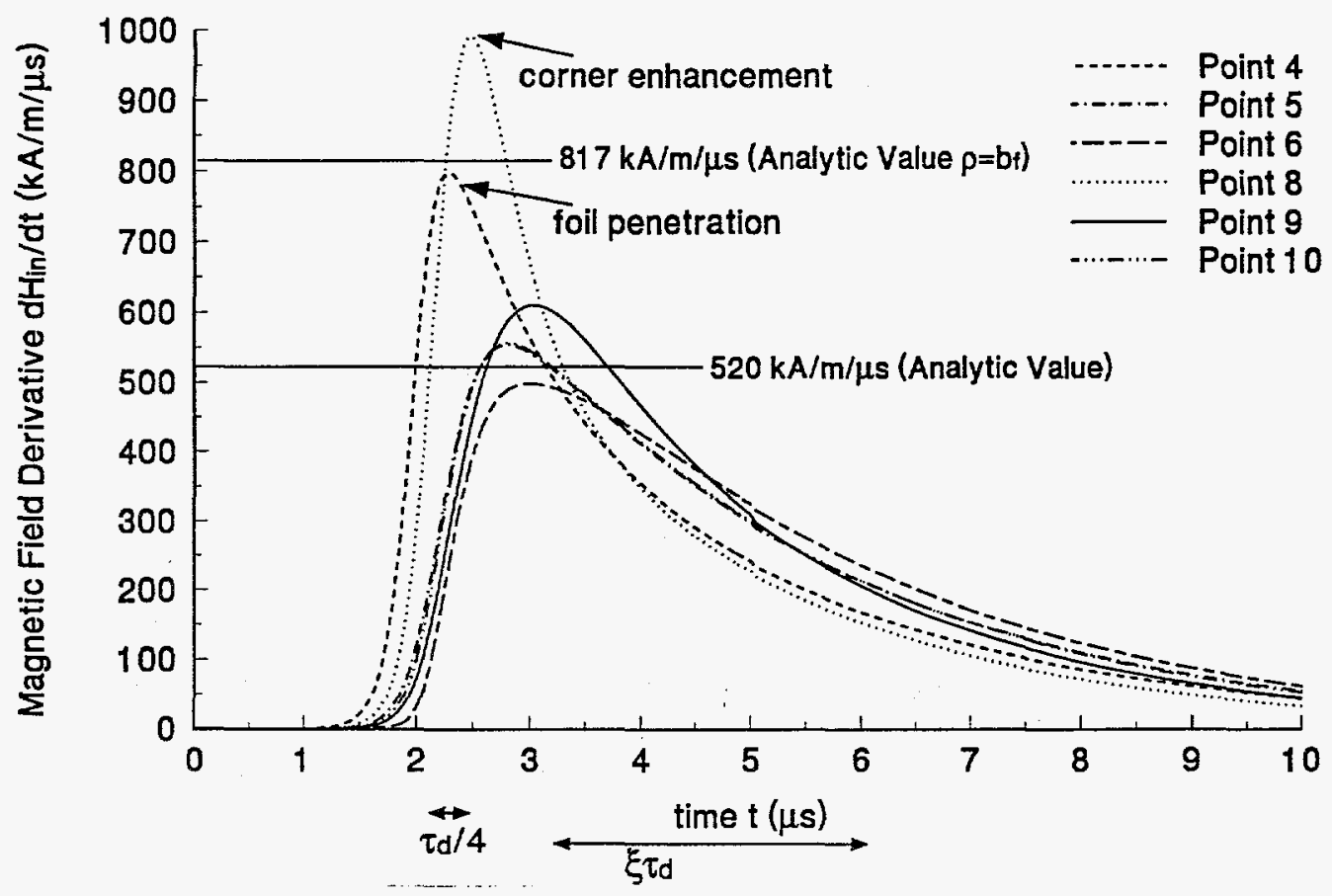

Penetrant Magnetic Field Derivative

Stainless Steel Enclosure, Air Core, $\mathrm{H}^{\text {inc }}=\mathrm{H} y=2.2 \mathrm{MA} / \mathrm{m}$

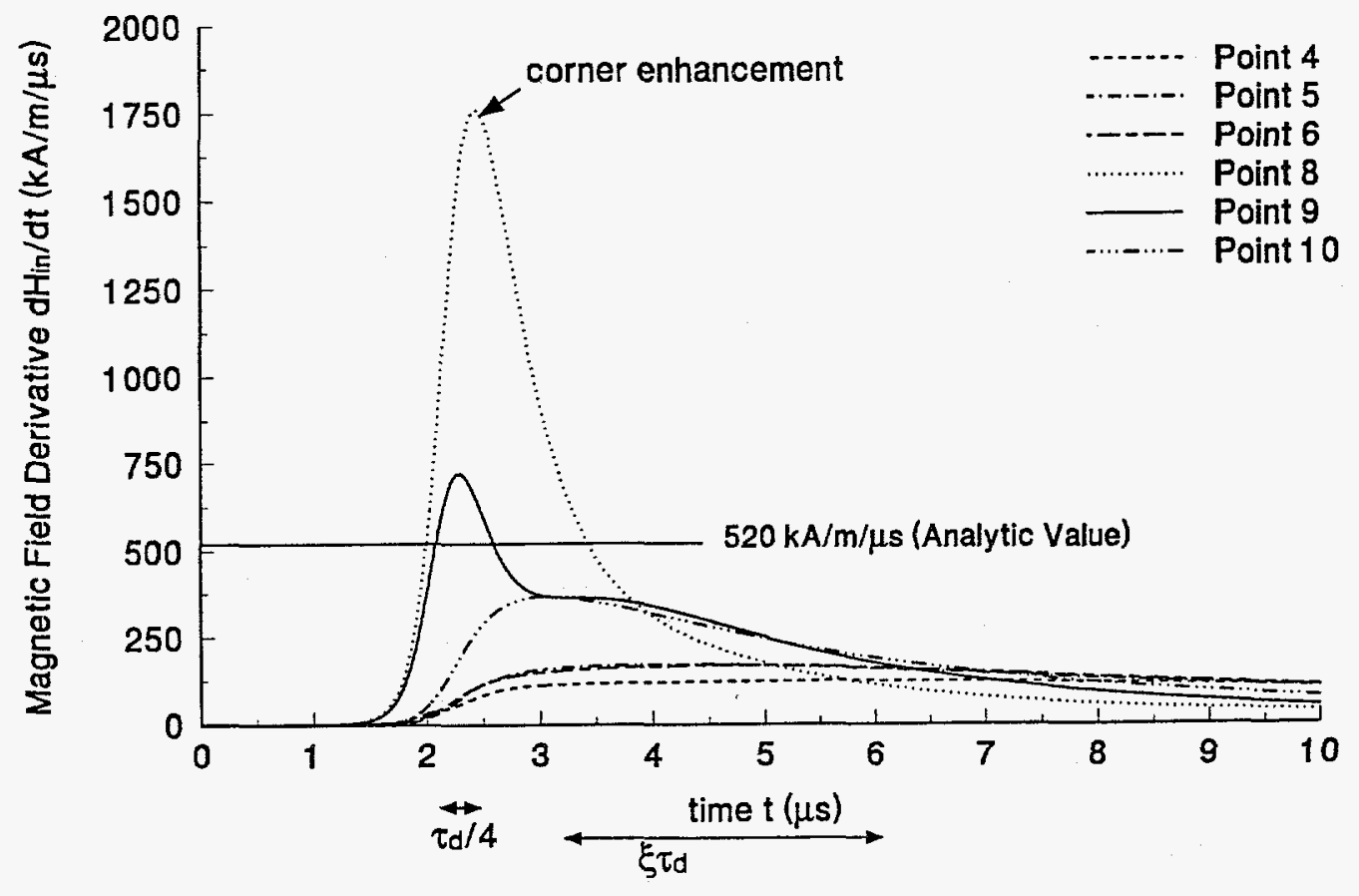

Figure 22. Summary of stainless steel air core finite element results for time derivative of total penetrant magnetic field. a) Incident $x$ polarization, b) Incident $y$ polarization. 


\section{Transformer Magnetic Flux per unit Length Aluminum Housing, PMC Core, $\mathrm{H}^{\text {inc }}=\mathrm{Hy}$}

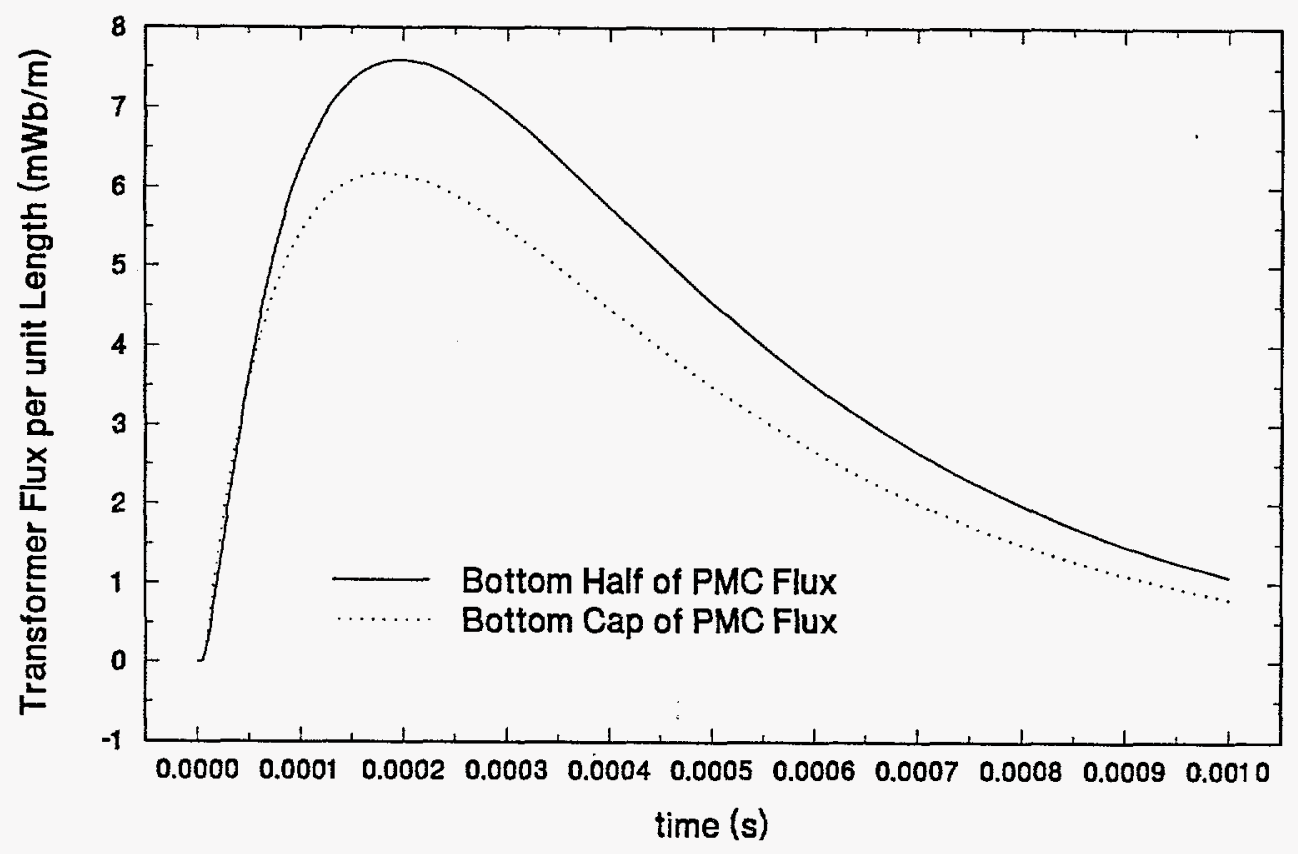

Time Derivative of Transformer Magnetic Flux per unit Length Aluminum Housing, PMC Core, $\mathrm{H}^{\mathrm{Hn}}=\mathrm{Hy}$

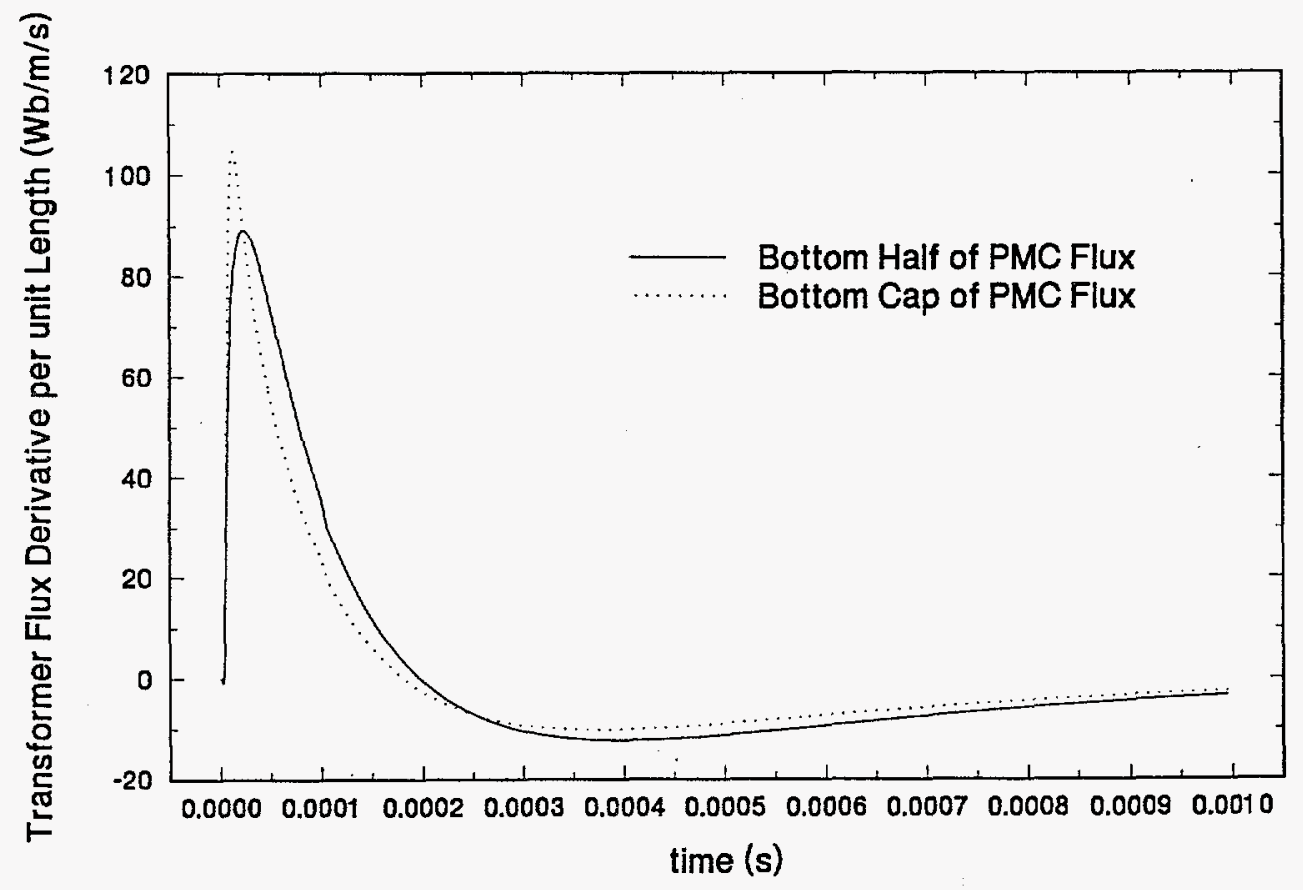

Figure 23. Finite element results for magnetic flux on bottom half of $\mathrm{PMC}$ core (point 5 to point 9 in Figure 11) and on bottom cap of PMC core (point 6 to point 8 of Figure 11) with aluminum pill box barrier. a) Total magnetic flux, b) Time derivative of magnetic flux. 


\section{Transformer Magnetic Flux per unit Length \\ Stainless Steel Housing, PMC Core, $\mathrm{H}^{\mathrm{inc}}=\mathrm{H}_{\mathrm{y}}$}

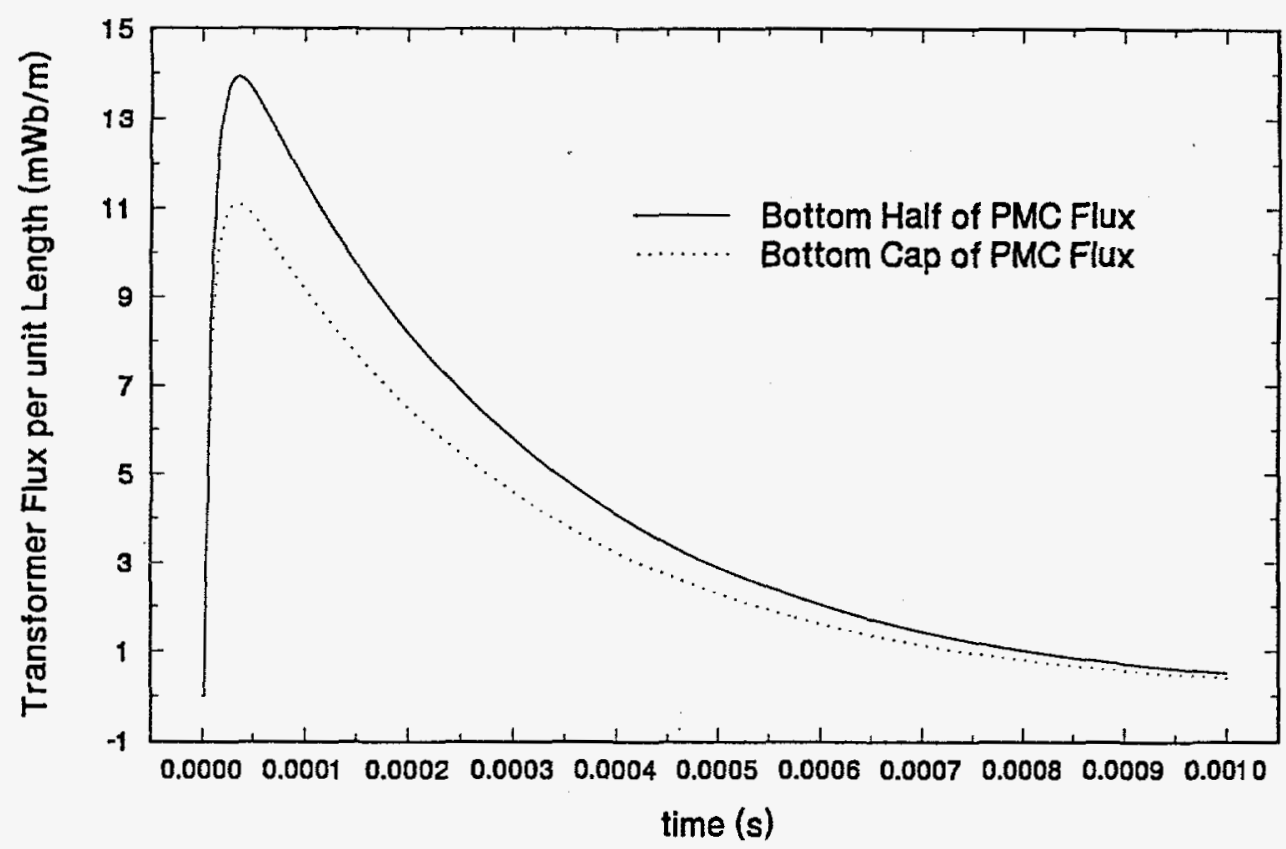

Time Derivative of Transformer Magnetic Flux per unit Length Stainless Steel Housing, PMC Core, $\mathrm{H}^{\mathrm{inc}}=\mathrm{Hy}$

है

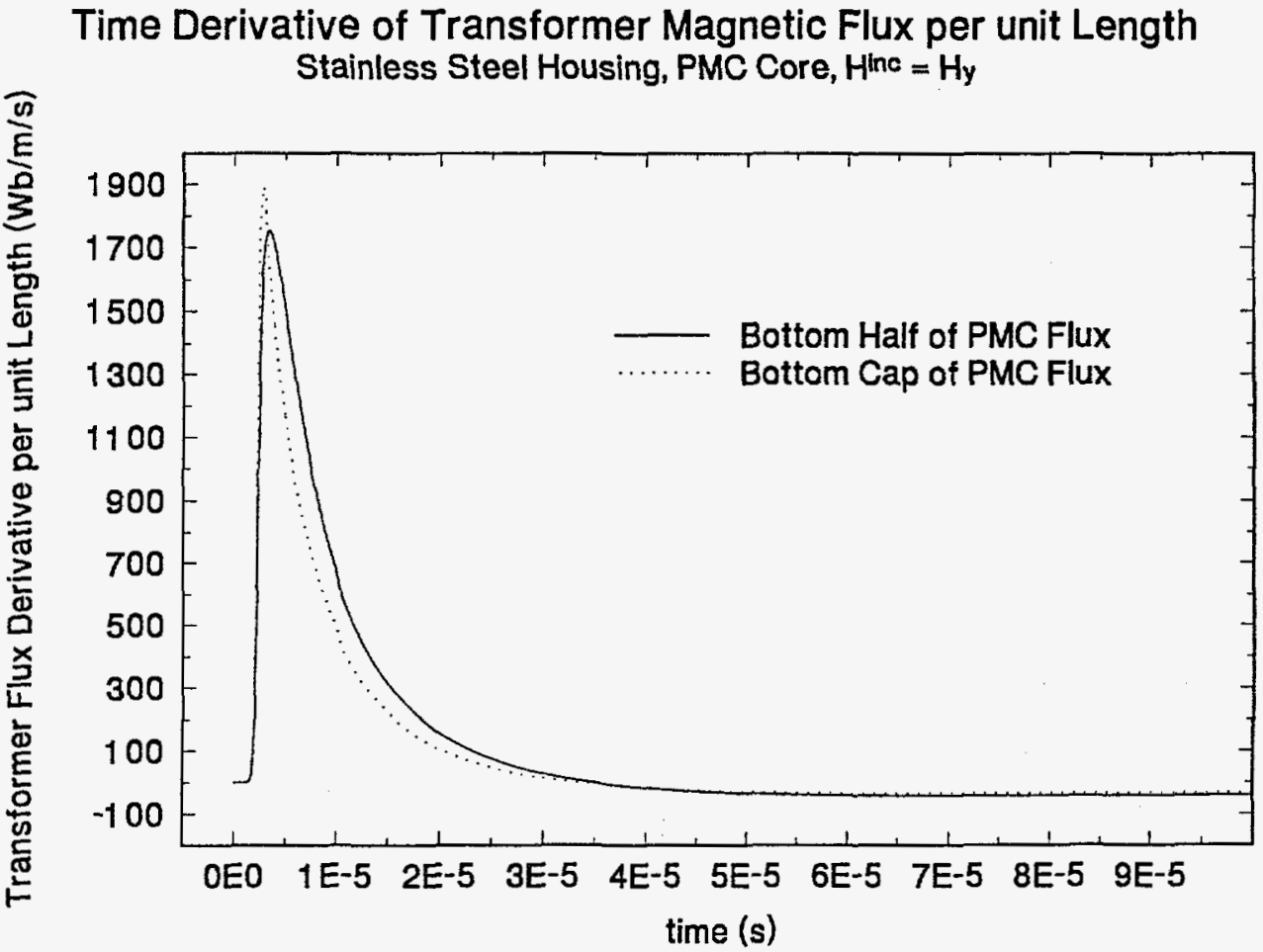

Figure 24. Finite element results for magnetic flux on bottom half of PMC core (point 5 to point 9 in Figure 11) and on bottom cap of PMC core (point 6 to point 8 of Figure 11) with stainless steel pill box barrier. a) Total magnetic flux, b) Time derivative of magnetic flux. 
The comparisons of the two dimensional finite element results of this section with previous analytical results have largely corroborated the analytical estimates. The time domain corner enhancement was nearly twice the value crudely estimated from Kaden's time harmonic results, however its effect was only observed at the corner and contributed only a small increase in the transformer flux derivative (which was still overestimated by the analytical flux derivative). The foil penetration estimate was corroborated by the stainless steel results (as much as was possible by two dimensional calculations). The enhancement of the $\frac{\partial}{\partial t} H_{y}$ value at point 9 (less than $50 \%$ greater than analytic estimate for stainless steel) was not observed at the center of the transformer, and appeared to be confined to the outer wall region. Thus the previous analytical estimates appear to be reasonably accurate.

\section{ENERGY CALCULATIONS}

The preceding sections of the report have focused on the induced voltage. The immediately preceding calculation used an energy argument in the solution of the detailed circuit model. This leads to an interesting alternative approach: instead of bounding induced voltage we can also attempt to bound the induced energy. Here we must be careful to remember that lightning typically consists of several return strokes and thus the statistical distribution for lightning must be called upon (the 1\% number of return stokes is 12 [3]). Furthermore, finite rise times and diffusion times, and resulting circuit response mismatches give reduced voltages; the following energy arguments ignore these mismatches and thus yield larger bounds on the induced energies.

Because of the long fall time we can consider step type excitation; the preceding results show that nearly the full exterior amplitude is reached by the penetrant magnetic field (particularly for the stainless steel barrier). The canonical problem is thus the energy stored in a loop or coil by a plane magnetic field, which can then be transferred to the capacitor.

A wire loop of radius $\rho_{\ell}$ and wire radius $a$ (with $\rho_{\ell}>>a$ ) has an inductance $L_{\ell}$ given by

$$
L \approx \mu_{0} \rho_{\ell}\left[\ln \left(8 \rho_{\ell} / a\right)-2\right]
$$

The magnetic flux in the loop resulting from a uniform field of amplitude $H_{0}$ is approximately

$$
\Phi \approx \pi \rho_{\ell}^{2} \mu_{0} H_{0}
$$

The short circuit current is thus

$$
I=\Phi / L_{\ell}
$$

The energy stored is thus

$$
W=\frac{1}{2} L_{\ell} I^{2} \approx \frac{1}{2} \mu_{0} H_{0}^{2} \frac{\pi^{2} \rho_{\ell}^{3}}{\ln \left(8 \rho_{\ell} / a\right)-2}
$$

This expression can also be written as

$$
W=\frac{1}{2} \mu_{0} H_{0}^{2} V M=\frac{1}{2} m \mu_{0} H_{0}=\frac{1}{2} m B_{0}
$$

where the magnetic flux density $B_{0}=\mu_{0} H_{0}$, the magnetic dipole moment of the loop is

$$
m=\pi \rho_{\ell}^{2} I=V M H_{0}=\frac{\pi^{2} \rho_{\ell}^{3}}{\ln \left(8 \rho_{\ell} / a\right)-2} H_{0}
$$

and $V M$ is the polarizability ( $V$ denotes volume of the loop region and $M$ the normalized polarizability). The next question is: if the loop has $N$ turns how are these formulas changed. The scaling 
is as follows: $L_{N} \approx N^{2} L, \Phi_{N} \approx N \Phi, I_{N} \approx I / N, W_{N} \approx W, m_{N}=\pi \rho_{\ell}^{2} N I_{N} \approx m$. Thus the energy is approximately independent $N$ (because the effective wire radius $a_{N}$ is larger for $N$ turns than for a single turn $a$ there is actually a small increase in the energy). Because the pill box provides little shielding as far as the maximum magnetic field level reached (particularly for stainless steel) we can use the exterior magnetic field to drive the loop.

Let us apply these formulas assuming a single turn loop (although taking $N=1$ is not important here) with $\rho_{\ell}=b \approx 13.5 \mathrm{~mm}, a \approx 0.1 \mathrm{~mm}, H_{0} \approx 2.2 \mathrm{MA} / \mathrm{m}$. The result for a single pulse is $W \approx$ $14.8 \mathrm{~J}$. This energy is far too large to rule out charging of the capacitor $\left(W_{\text {out }}=\frac{1}{2} C_{\text {out }} V^{2} \approx 18.4\right.$ $\mathrm{mJ})$.

Second, we apply the energy argument to the pot core transformer. The transformer core is nonlinear and thus we expect to have to modify the energy formula to $W=\int m d B$. However since the saturation flux of the ferrite core is less than $B_{s}<0.5 \mathrm{~T}$, and the driving magnetic field is so large that $\mu_{0} H_{0}=2.8 \mathrm{~T}$, we conclude that the core will be driven to complete saturation, and furthermore we can approximately ignore the saturation flux and take the winding to be an air core. Because the core consists of many windings $N=400$ we again use the solenoid formula (74) and

$$
W=\frac{1}{2} \Phi^{2} / L_{w} \text { where } \Phi \approx N \pi b_{w}^{2} \mu_{0} H_{0}
$$

The energy is thus $W \approx 0.253 \mathrm{~J}$ for a single pulse. These energy calculations thus do not yield useful results in ruling out capacitive charging. It should be kept in mind that the energy calculations in this section would give the same results for a very long rise time field, which would obviously contribute very little to the capacitor energy (since very little voltage would be induced).

\section{OUTER SHIELD WALL}

Because we were not entirely successful in ruling out the lightning threat by means of the previous induced voltage calculations (at least for stainless steel), let us consider the presence of another planar shield wall exterior to the pill box. Let us take this shield to have the electrical properties of stainless steel and thickness $\Delta_{s} \approx 3 \mathrm{~mm}$. Only the stainless steel pill box barrier results will be considered in this section. The diffusion time (10), with $\Delta$ replaced by $\Delta_{t o t} \approx 4 \mathrm{~mm}$ (the sum of the outer shield wall thickness and inner pill box wall thickness), increases by a factor of 16 to $\tau_{d} \approx 28 \mu \mathrm{sec}$. The worst case induced voltage (44) also shows a reduction by a factor of 16 from the result (46) to 61 volts. The more practically attainable result (49), now using $\Delta_{\text {tot }}, \rho_{0}^{\text {(close) }}=6$ $\mathrm{mm}$, and $\rho_{0}^{(f a r)}=9 \mathrm{~mm}\left(\Delta_{s}\right.$ was added to the previous values), gives only

$$
V \approx 34 \text { volts }
$$

for stainless steel (even this value is thought to be an overestimate, since the inner shield wall is much thinner than the outer wall, and thus the doubling of the field used in this formula is an overestimate). If this voltage were induced in the primary circuit, this value times the turns ratio 40 is just above the threshold. (A flyback transformer can impart a greater than turns ratio step up; an effect which should be assessed with the resulting slower rising $\tau_{d} / 4 \approx 7 \mu$ sec and falling $O\left(\tau_{d}\right)$ interior magnetic field in this case.)

To estimate the direct coupling to the transformer in this case, we consider the case (which is believed to be an upper bound) of a pill box with the same interior volume and surface area, but with a wall thickness $\Delta_{t o t}$. Using very approximate linear interpolation from the graph in [6] we find $(13)$ is approximately equal to 0.34 with $\xi \approx 1.55 / 4$. Thus we find for stainless steel a peak 
value $\frac{\partial}{\partial t} H_{i n}^{\text {step }} \approx 6.9 \times 10^{10} \mathrm{~A} / \mathrm{m} / \mathrm{sec}$. If we use $(72)$ for the open circuit secondary transformer voltage, we find for stainless steel

$$
V \approx 576 \text { volts }
$$

which is smaller than the threshold. The thicker wall (the vertical spacing from the lightning current to the center of the transformer is $1 \mathrm{~mm}+4 \mathrm{~mm}+2 \mathrm{~mm}=7 \mathrm{~mm}$ ) means that the magnetic field strength $(7)$ (arrived at with a line source separation of $14.5 \mathrm{~mm}$ ) is an upper bound $\left(H_{0} \approx\right.$ $\frac{I_{0}}{(7 \sqrt{2} \mathrm{~mm}) \sqrt{2}}$ compared to $\frac{I_{0}}{14.5 \mathrm{~mm}}$ for vertical late time magnetic field at center of transformer). The line source result (73) in this case gives a field derivative $\frac{\partial}{\partial t} H_{y}^{\text {step }} \approx 1.2 \times 10^{11} \mathrm{~A} / \mathrm{m} / \mathrm{sec}$. Applying this time derivative in (72) gives

$$
V \approx 1 \mathrm{kV}
$$

This value is slightly above threshold, however it must be kept in mind that the presence of the bottom wall of the pill box will reduce the vertical field and that the thin sheet impedance solution is thought to overestimate the true field derivative.

The worst case open circuit voltages with the outer shield wall present that have been calculated are thus slightly above threshold. It is believed that the overestimates inherent in these simple calculations put the true worst case voltages at the threshold level, and thus the outer shield wall mitigates the lightning threat. If it is of interest, some further calculations could be done in the short term to better quantify the actual worst case solutions.

\section{POWER LINE CURRENT AND FIELD}

Finally we consider the power line threat. The accident would involve the shorting of a power line in the immediate vicinity of the pill box barrier. A requirement for power line threats to weapons is currently being debated and thus this section cannot be the final verdict on this threat. Nevertheless, it is possible to give some guidance on this issue.

The form of the power line in the vicinity of the accident will consist of either twin wire leads or one lead with respect to ground and will have a characteristic impedance of above about 60 ohms. (Note that the characteristic impedance of a wire of radius $a$, a height $h_{p}$ above a ground plane, has characteristic impedance $Z_{c}=60 \mathrm{ohms} \operatorname{Arccosh}\left(h_{p} / a\right)$.) High voltage power lines range up to 750 kilovolts. Therefore a peak current level of $I_{0} \approx 2(750$ kilovolts $\mathrm{rms}) \sqrt{2} / 60 \mathrm{ohms} \approx 34 \mathrm{kA}$ appears to be reasonable worst case level. The rise time of the $60 \mathrm{~Hz}$ waveform (4.2 msec quarter period) is too long to be of concern (the rise rate would be very small). However, an arc established as a result of the accident may introduce fast transients. If we take the rise time to be comparable to the lightning current waveform we see that the factor of 6 reduction in peak amplitude results in a less severe threat.

\section{CONCLUSIONS}

The problem was set up as a conducting barrier surrounding circuitry, consisting of a flyback transformer with 40 to 1 turns ratio, with pick-up wire loops for magnetic flux coupling in both the primary and secondary circuits of the transformer. The secondary circuit consists of a diode rectifier and an output capacitor with threshold charge voltage of 700 volts and threshold charge energy of $18.4 \mathrm{~mJ}$ (the no-go levels are $V_{\text {out }}=500 \mathrm{volts}, W_{\text {out }}=9.4 \mathrm{~mJ}$, which are also important for comparison in certain safety studies). The question then posed was: can penetrant magnetic fields, resulting from lightning currents or power line currents in the vicinity of the shield, induce voltages or energies that are large enough to be of concern. The purpose of the report was thus 
to assess the fields interior to the shield and give upper bound estimates for the resulting induced voltages and energies.

A brief discussion was given about the statistical distributions of the lightning parameters [3], [4], and [5]. The important parameters in the present problem are peak amplitude, maximum fall time, peak rise rate, and number of return strokes per lightning flash. It is common practice to use the $1 \%$ worst case set of parameters for safety studies. Thus an inverse exponential waveform (4) was fit by means of the three parameters $(200 \mathrm{kA}$ peak current, $200 \mu \mathrm{sec}$ fall time to half peak, and a maximum rise rate of $400 \mathrm{kA} / \mu \mathrm{sec}$ ) to represent the lightning current in the present study. Taking this current to be on a thin filament, the azimuthal magnetic field surrounding the filament is given by (6). Two cases were treated: simplified uniform field excitation, where the field amplitude was given by (7), corresponding to a filament distance of one pill box shield radius (for which the uniform field approximation is reasonable), and second, worst case wire excitation, where the entire lightning current is carried by a thin wire near the top surface of the pill box shield (the direct transformer winding excitation is considered with the amplitude (7) as well as a closer spacing, which is thought to be the worst case, yielding twice this amplitude).

Existing results, describing plane field penetration of very short electromagnetic pulse fields [6], which are approximated by an impulse waveform, were used to estimate the time derivative of the penetrant field resulting from step function excitation (step function excitation is a reasonable approximation for lightning magnetic fields and provides an upper bound). The penetrant field time derivative is thus given by (15). Taking an assumed worst case wire pick-up single turn loop to have an area of the interior shield height times the interior shield diameter gives the voltage results (18). These results are too small to be of concern in the secondary output circuit, however the behavior of the transformer with these input voltages (at least with a stainless steel shield) needs to be considered. The existing analytical results [6] (which are based on smooth canonical shapes) for the penetrant magnetic field did not include three effects that are present here: the shield has sharp corners, the permeable transformer fills the interior height of the shield (perhaps loading it), and there is a central thin foil in the bottom shield wall.

High frequency time harmonic results from [8] for the corner enhancement of penetrant fields, were used to argue that the time domain field derivative enhancement is not large (order of twice the nominal interior penetrant field derivative), and that the enhancement only exists local to the corners and is not expected to be a significant contributor at the circuit loops or for direct transformer coupling.

The time harmonic solution of a cylindrical shield filled with highly permeable material was compared to the corresponding solution filled with free space $\mu_{0}$ material to show that there was no significant high frequency (early time) enhancement of the penetrant fields. Thus the rise rate of the penetrant field is not expected to be significantly affected by the transformer loading.

The central foil penetration was addressed by use of impedance loaded aperture theory [6]. The foil is thin enough that the diffusion times associated with it are small compared to the exterior field rise time, which makes the impedance approximation applicable. The impedance loading may be ignored for the stainless steel barrier since the time constant associated with the impedance loading is shorter than the exterior field rise time. The interior penetrant fields of the open hole can then be approximately represented by a line magnetic dipole (not too near the hole). Evaluating the time derivative of these fields at twice the hole radius $(7 \mathrm{~mm}$ ) (this location is reasonable for circuit loop locations and is near the inner edge of the transformer) gives (33) which is less than half the nominal interior value (15), and in the absence of perturbing obstacles, is horizontally oriented with respect to the circuit boards. The foil penetration thus does not appear to be a major factor for stainless steel. The aluminum shield foil penetration is treated by means of the loaded aperture theory and step function excitation. The result for the aluminum foil penetration, at the same 
location, is (36) which is somewhat larger than twice the nominal value (15). Nevertheless because of the horizontal field orientation, in addition to the overall smaller penetrant values for aluminum, this appears not to be of significant importance. It should be kept in mind that the nearby wire excitation is not a factor in the foil penetration mode since the lightning current is constrained to be near the top surface of the pill box. Thus the exciting field amplitude at the foil will never be greater than the value (7) whereas the diffusive penetration of the thick walls of the barrier does increase for the nearby wire excitation discussed next.

The worst case wire excitation (lightning current on exterior surface of shield wall and wire pick-up on adjacent interior surface of shield wall) [11], with a pick-up loop of one interior shield diameter in length, gives the voltage results (46). The stainless steel result is just above threshold even in the secondary output circuit. Making these results more realistic by using more reasonable spacings of $1 \mathrm{~mm}$ from the outer wall to the centroid of the lightning current and $1 \mathrm{~mm}$ from the inside wall to the pick-up-loop (which runs the length of the interior shield diameter and has return on the opposite bottom wall of the shield) gives the voltage results (49). These more realistic worst case results are below threshold when applied in the output or secondary circuit. If, in the stainless steel case, this voltage is applied in the primary circuit, there is still a question about the performance of the transformer with a maximum of twelve such pulses (the $1 \%$ worst case number of return stokes [3]). (Normal operation typically requires several thousand pulses.)

Direct magnetic field coupling to the transformer was briefly considered. If the highly permeable transformer is placed in a uniform field, the magnetic flux that impinges on the bottom half is approximately twice the bottom plate area (this was estimated by modeling the transformer as an oblate spheroid (59)). Because of the air gap in the center of the pot core transformer, most of the magnetic flux passes through the outer cylindrical shell and does not link the windings. Using the penetrant field excitation (15), and the initial permeability of the ferrite material, this effect is illustrated by the output voltages (in the 400 turn secondary) given by (70). However, because the lightning fall time is sufficiently large (particularly for stainless steel) compared to the barrier diffusion times, the penetrant field nearly reaches the same level as the exterior field. Since the lightning penetrant magnetic field is therefore so large that it fully saturates the ferrite material of the transformer, we cannot rule out significant flux coupling to the transformer windings. If we consider the output winding as an air core with free space magnetic permeability, and use the interior field derivative (15), we obtain the open circuit voltage (72). This open circuit voltage is well above threshold for the stainless steel barrier. Furthermore, as a worst case, for closer wire excitation than represented by the levels (15), we could see twice this level, as discussed below (72).

Detailed circuit modeling of the transformer and attached loads is beyond the scope of this report. However it is believed that this will reduce the stainless steel open circuit voltage threats (and could conceivably allow dismissal). A simple example was treated to illustrate how this can work. It was assumed that the primary of the transformer was shorted. However there appears to be sufficient leakage flux in the secondary winding (discounting the central core area which has shorted primary winding, and assuming that the outer shell of the transformer has been fully saturated) to allow the open circuit voltage to appear in the secondary circuit. But a secondary free space leakage inductance will also appear. With 400 turns, this leakage inductance is not trivial (here it was very crudely estimated by means of a solenoid in free space). A circuit consisting of this leakage inductance, the open circuit voltage, and the output capacitor gives the charge energy (77), which is still somewhat above threshold, but reduced from the open circuit voltage charge energy. The reduction in energy is caused by the significant charge time of the inductor-capacitor circuit. It is possible if more realistic loads are used that this energy can be further reduced.

A two dimensional finite element model was used to model the pill box barrier, and also high permeability and low (saturated) permeability approximations to the transformer. The results as 
a whole corroborated previous analytical estimates for the penetrant fields (exceptions: up to a factor of four enhancement in penetrant field was noted at the corners versus the previous crudely estimated factor of two, and up to a factor of two enhancement in penetrant field was noted against the outer cylindrical wall of the shield). The derivative of the magnetic flux coupled into the high permeability transformer was shown to be somewhat overestimated by the analytical results (less than a factor of two).

Using the interior penetrant field (which is nearly equal to the exterior field for stainless steel) the energies stored in loops and in the transformer windings were estimated. These results are above energy thresholds. This approach does not take into account impedance mismatches (for example, a very slowly rising field would result in the same energy bounds even though no significant voltages would be induced).

The effect of an extra shield wall was considered. The planar wall was taken as a $3 \mathrm{~mm}$ thick stainless steel shield and considered only in conjunction with the stainless steel pill box (since the aluminum results were already small). The addition of this thickness to the pill box, results in a wall four times as thick and thus an increase in the diffusion time by a factor of sixteen. A calculation of the reduction in the nearby line source coupling to an interior wire loop (49) resulted in a factor of twelve reduction to 34 volts (91). The direct coupling to the transformer by a uniform field was reduced from (15) and (72) by a factor of 7.5 to 576 volts (92). The worst case line source coupling to the transformer was reduced by a factor of 12.5 to $1 \mathrm{kV}(93)$. The effect of the slowly varying penetrant field (with the outer shield wall $\tau_{d} / 4 \approx 7 \mu \mathrm{sec}$ ) on the flyback transformer still needs to be assessed. Nevertheless, these results which are near threshold (and noting that these simple estimates overestimate the true worst case open circuit voltages), indicate that the outer shield wall mitigates the lightning threat. (If it is of interest, some further calculations can be done in the short term to better quantify the actual values.)

Power line threats are currently being investigated to determine an appropriate specification for safety studies. Nevertheless, discussions in this report indicate that the power line is less severe than lightning and is thus covered by previous analyses.

The results provided in this report for the field derivatives (and open circuit voltage drives) can be used with more detailed interior circuit models and transformer models to obtain better (and probably reduced) estimates of the output capacitor charge voltage. The understanding of the diffusive penetrations can also be used to set up an effective series of tests to measure the worst case capacitive charge voltages.

\section{ACKNOWLEDGEMENT}

The authors would like to thank M. J. De Spain, of Sandia National Laboratories, for initiating this study and for his support.

\section{References}

[1] R. C. Weast (editor), CRC Handbook of Chemistry and Physics, Boca Raton: CRC Press (66th edition), 1985, p. D-184.

[2] T. Lyman (editor), Metals Handbook, Vol. 1, American Society for metals, 1961, p. 946.

[3] N. Cianos and E. T. Pierce, A ground-lightning environment for engineering usage, TS3141/7723, Menlo Park: Stanford Research Institute, Aug. 1972.

[4] M. A. Uman, The Lightning Discharge, New York: Academic Press, Inc., 1987, pp. 124-125.

[5] R. J. Fisher and M. A. Uman, "Recommended Baseline Direct-Strike Lightning Environment for Stockpile-to-Target Sequences," Sandia National Laboratories Report, SAND89-0192, May 1989 . 
[6] K. S. H. Lee (editor), EMP Interaction: Principles, Techniques, and Reference Data, New York: Hemisphere Pub. Corp., 1986, pp. 302-306, 454-458, 519-521, 559-571.

[7] L. K. Warne, K. O. Merewether, K. C. Chen, M. E. Morris, J. E. Solberg, and J. G. Lewis, "Validation of EMP Bounds," Sandia National Laboratories internal memorandum, September $30,1995$.

[8] H. Kaden, Wirbelstrome und Schirmung in der Nachrichtentechnik, Berlin: Springer-Verlag, 1959, pp. 106-123.

[9] W. A. Johnson, L. K. Warne, K. C. Chen, and E. M. Gurrola, "Linear Diffusion and Internal Voltages in Conducting Enclosures Subjected to a Direct Lightning Strike," Electromagnetics, 15: 189-207, 1995.

[10] L. K. Warne, W. A. Johnson, and K. C. Chen, "Nonlinear Diffusion and Internal Voltages in Conducting Ferromagnetic Enclosures Subjected to Lightning Currents," IEEE Transactions on Electromagnetic Compatibility, Vol. 37, No. 2, May 1995, pp. 145-154.

[11] L. K. Warne, W. A. Johnson, and K. C. Chen, Maximum interior voltage and magnetic field penetration through a linear conducting layer, Journal of Electromagnetic Waves and Applications, Vol. 9, No. 4, 1995, pp. 569-598.

[12] W. R. Smythe, Static and Dynamic Electricity, New York: Hemisphere Pub. Corp., 1989, pp. 168-171, 232.

[13] E. Weber, Electromagnetic Theory, New York, Dover Pub., 1965, pp. 501-503.

[14] J. A. Stratton, Electromagnetic Theory, McGraw-Hill Book Co., 1941, pp. 205-207.

[15] Phillips, Ferrite Materials and Components Catalog, eighth edition.

[16] S. Chikazumi, Physics of Magnetism, Krieger Pub. Co., 1978, pp. 24-29.

[17] Chih-Wen Chen, Magnetism and Metallurgy of Soft Magnetic Materials, New York: Dover Pub., Inc., 1986, pp. 400-403.

[18] F. W. Grover, Inductance Calculations, Dover Pub. Inc., 1946, pp. 142-148. 


\section{Distribution}

8 Los Alamos National Laboratory

Attn: G. A. Buntain, MS C920

B. P. Ginsberg, MS P950

M. D. Holder, MS P950

E. Martinez, MS P950

D. Montoya, MS P950

S. G. Mozzano, MS P950

J. D. Paul, MS P950

R. J. Yactor, MS P950

P.O. Box 1663

Los Alamos, NM 87544

1

1
MS 9015

MS 9036

MS 9036

MS 9018

MS 0311

MS 0311

MS 0311

MS 0328

MS 0328

MS 0328

MS 0445

MS 0445

MS 0445

MS 0490

MS 0492

MS 0492

MS 0492

MS 0523

MS 0865

MS 0865

MS 0865

MS 0865

MS 0865

MS 0865

MS 1166

MS 0899

MS 0619

MS 0100
E. Talbot, 2221

D. Gehmlich, 2254

M. Neuman, 2254

Central Technical Files, 8523-2

J. W. Hole, 2671

D. M. Pierce, 2671

M. J. De Spain, 2671

P. A. Smith, 2674

J. H. Weinlein, 2674

J. A. Wilder, 2674

O. Brooks, Jr., 2166

R. C. Hartwig, 2166

M. G. Orrell, 2166

J. A. Cooper, 12331

R. D. Pederson, 12324

D. H. Loescher, 12332

J. F. Wolcott, 12332

R. L. Nagel, 2251

M. E. Morris, 9753

K. C. Chen, 12332

W. A. Johnson, 9753

R. E. Jorgenson, 9753

K. O. Merewether, 9753

L. K. Warne, 9753

J. D. Kotulski, 9352

Technical Library, 4414

Print Media, 12615

Document Processing, 7613-2 for DOE/OSTI 